Supplemental Information for:

Bioinformatic mapping of radical SAM-dependent RiPPs identifies new $\mathrm{C} \alpha, \mathrm{C} \beta$, and $\mathrm{C} \gamma$-linked thioether-containing peptides

Graham A. Hudson $^{\dagger \S}$, Brandon J. Burkhart ${ }^{\dagger \S}$, Adam J. DiCaprio ${ }^{\dagger \dagger}$, Christopher J. Schwalen ${ }^{\dagger}$, Bryce Kille ${ }^{\dagger}$, Taras V. Pogorelov ${ }^{\dagger \uparrow} \perp / /$, Douglas A. Mitchell ${ }^{\dagger * *}$

${ }^{\dagger}$ Department of Chemistry, ${ }^{\dagger}$ Carl R. Woese Institute for Genomic Biology, ${ }^{\top}$ Center for Biophysics and Quantitative Biology, ${ }^{\S}$ School of Chemical Sciences, ${ }^{\perp}$ National Center for Supercomputing Applications,

"Beckman Institute for Advanced Science and Technology, University of Illinois at Urbana-Champaign, Urbana, Illinois 61801, USA.

$\S$ These authors contributed equally

* Corresponding author

DAM: Phone: 1-217-333-1345; Fax: 1-217-333-0508; E-mail: douglasm@illinois.edu

Table of Contents

Supplemental Methods 3-8

Table S1. Primers used in this study......

Table S2: Top 250 most common pfams found within 8 orfs of a sactipeptide-like rSAM. 10-11

Table S3: ${ }^{1} \mathrm{H}$ Chemical shift values of assigned huazacin residues........................................................ 12

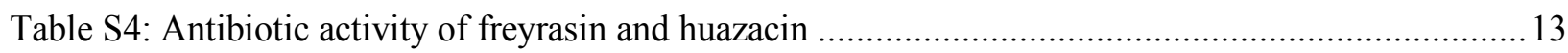

Table S5: ${ }^{1} \mathrm{H}$ Chemical shift values of assigned freyrasin residues................................................... 14

Table S6: MS/MS Expected and observed masses of diagnostic ions.......................................... 15-17

Table S7: Identity matrix of characterized rSAMs involved in RiPP thioether formation ...................... 18

Figure S1: Bioinformatic analysis of sactipeptide precursor peptides................................................ 19

Figure S2: MALDI-TOF-MS of B. thuringiensis serovar huazhongensis extracts. ...............................20

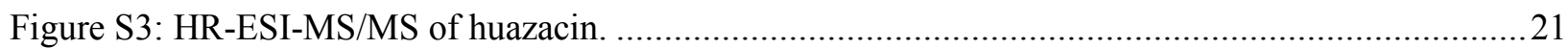

Figure S4: Amide-side chain 1H-TOCSY correlations and assignments of huazacin............................22

Figure S5: NH-NH NOESY correlations of backbone amides of huazacin .........................................23

Figure S6: NOESY correlations supporting the S-C $\alpha$ thioether linkage of huazacin.............................24

Figure S7. TOCSY correlations of the H-C $\alpha$ region of huazacin....................................................2 25

Figure S8: Abundance and sequence characteristics of annotated precursor peptides ...........................26

Figure S9: Bioinformatic analysis of SCIFF/ranthipeptide precursor peptides...................................2 27

Figure S10: Phylogenetic analysis of organisms containing sactipeptide and SCIFF gene clusters.........28

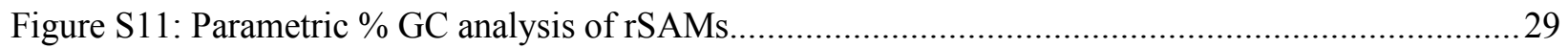

Figure S12: Unusual genomic contexts of SCIFFs................................................................... 30

Figure S13: Maximum likelihood tree of rSAMs involved in thioether formation..................................31 
Figure S14: Trypsin digest of the freyrasin precursor peptide.

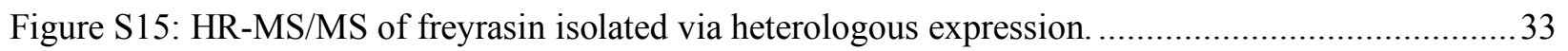

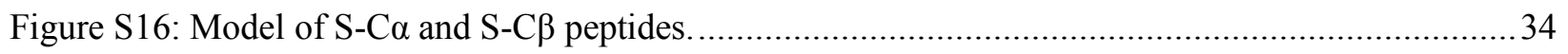

Figure S17: Removal of MBP tag and N-terminal residues from expressed freyrasin........................... 35

Figure S18: Amide-side chain 1H-TOCSY correlations and assignments of freyrasin............................36

Figure S19: NH-NH NOESY correlations of backbone amides of freyrasin......................................... 37

Figure S20: Amide proton-side chain correlations of S-C $\beta$ linked Asp residues of freyrasin .................. 38

Figure S21: Overexpression of Thr-labeled CteA with Ile and Gly supplementation.............................. 39

Figure S22: MS/MS localization of isotopic labels to Thr in labeled CteA.......................................... 40

Figure S23: MALDI-TOF-MS analysis of labeled CteA coexpressed with CteB............................... 41

Figure S24: MS/MS analysis of unmodified CteA containing isotopically-labeled Thr .........................42

Figure S25: MS/MS analysis of modified CteA containing natural abundance Thr. ............................ 43

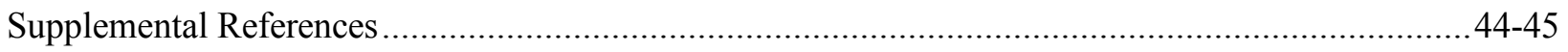




\section{Supplemental Methods}

General. Materials and reagents were purchased from Gold Biotechnology, Fisher Scientific, or SigmaAldrich unless otherwise noted. Molecular biology reagents for cloning (e.g. restriction enzymes, Q5 polymerase, T4 DNA ligase, and deoxynucleotides) were purchased from New England Biolabs. DNA primers were obtained from Integrated DNA Technologies (Table S1). DNA spin columns were purchased from Epoch Life Sciences. Sanger DNA sequencing was performed by the Roy J. Carver Biotechnology Center (University of Illinois at Urbana-Champaign). Polymerase chain reactions were performed using a Bio-Rad S1000 thermal cycler.

Bioinformatic mining of Cys-rich rSAM-modified peptides. To identify potential radical SAM (rSAM)modified, thioether-linked peptides, PSI-BLAST ${ }^{1}$ searches were performed in late 2018 with the four known sactipeptide rSAM proteins (AlbA, WP_003242599.1; SkfB, NP_388073.2; ThnB, ACY56718.1; and TrnC, AED99784.1) $)^{2}$ and two partially characterized SCIFF rSAM proteins (CteB ${ }^{3}$, WP_003517268.1; and Tte1186 ${ }^{4}$, AAM24417.1) for four iterations with a stringent PSI-BLAST threshold of 1e-70 to populate a list of related enzymes. Truncated enzyme records were removed by eliminating sequences less than 300 amino acid yielding a final list of $(n=4,693)$. Cysteine-rich $(\geq 3$ Cys residues) precursor peptide candidates encoded near these rSAMs were found using our automated ribosomally synthesized post-translationally modified peptide (RiPP) genome mining platform, Rapid ORF Description \& Evaluation Online (RODEO). RODEO output may be found in Supplementary Dataset 1. While this procedure yielded an illustrative dataset for sactipeptide- and SCIFF-/ranthipeptide-related rSAMs, it does include the nearly 40,000 unique sequences available in GenBank that occupy the sequence space between the two classes. Therefore, caution is warranted in comparing the similarity of sactipeptide from ranthipeptide synthases (i.e. they appear more closely related owing to the omission of $\sim 40,000$ other sequences).

New release of RODEO. RODEO 2.0 is a rewritten and more efficient version of the previous RODEO algorithm. ${ }^{5}$ The new version follows the same logic as the previous version by obtaining/analyzing the genomic region neighboring a gene(s) of interest while also prospecting for potential RiPP precursor peptides. RODEO 2.0 was written in Python and retrieves protein or DNA FASTA files from GenBank using the NCBI E-Utilities (Entrez Direct) ${ }^{6}$ embedded in Biopython. ${ }^{7}$ The source code and tutorials for local installation and use of RODEO are available at: http://www.ripprodeo.org. A web-based tool is also available for smaller jobs from infrequent users. The major functional improvement in RODEO 2.0 is that each step of the program has its own dedicated module which greatly increases the efficiency of altering specific steps within the program. This means that the peptide scoring algorithm for identifying additional classes of RiPP precursor peptides are more easily incorporated into the RODEO platform. Currently, RODEO 2.0 is capable of scoring RiPP precursor peptides for the following four classes: lanthipeptides, sactipeptides, lasso peptides, and thiopeptides.

Precursor identification. Precursor peptide prioritization was based on the RODEO scoring module developed for sactipeptides (https://antismash.secondarymetabolites.org and http://www.ripprodeo.org). ${ }^{8}$ While our efforts uncovered that the majority of Cys-rich peptides encoded adjacently the rSAM enzymes were not strictly sactipeptides, related classes of precursors could reliably be found using metrics developed for this class. To account for intrinsic differences between identified Cys-rich peptides and canonical sactipeptides for which the original scoring was originally optimized, a lower threshold of $\geq 10$ was employed (instead of the $\geq 25$ cutoff used to identify sactipeptides). This relaxed list of precursor peptides was then manually curated to afford a final list of non-redundant precursor sequences $(n=2,145$; comprised of 504 sactipeptides and 1,634 members of the SCIFFs) which were used to seed the precursor peptide sequence similarity networks (Supplemental Dataset 2).

Sequence-similarity network (SSN) generation. The predicted precursor peptide SSNs (Figures S1 and S9) were generated using EFI-EST (http://efi.igb.illinois.edu/efi-est) using the "representative node 90" 
parameter to conflate sequences that are $>90 \%$ identical to a single node. The resulting network was visualized in Cytoscape 3.4.0 using the organic layout and annotated with RODEO output. ${ }^{9,10}$ Sequence logos of the corresponding clusters of precursor peptides were generated using WebLogo 2.8.2 (http://weblogo.berkeley.edu/logo.cgi) for the major clusters present in the precursor SSNs. The SSN for the rSAM enzymes (Figure 2) was generated using EFI-EST and the protein list acquired by PSI-BLAST of the known sactipeptide synthases and two partially characterized SCIFF rSAM enyzmes. An alignment score of 50 and "representative node 70" parameter were chosen to conflate sequences $>70 \%$ identical. The resulting network was visualized in Cytoscape. ${ }^{10}$ Color-coding of nodes was based on identified precursor peptide or biosynthetic pathway identified. Sactipeptide and SCIFF precursors were identified by RODEO and manually curated. SCIFF precursor peptide mining was also aided by identifying matches to the SCIFF pfam (PF13165) using hmmr3.0 (http://hmmer.org). ${ }^{11}$ Accessions involved in quinohemoprotein amine dehydrogenase maturation were identified by the local presence of proteins matching pfam models PF08992, PF09098, PF09099, PF09100, or PF14930. ${ }^{12}$

Preparation of huazacin. Bacillus thuringiensis serovar huazhongensis was obtained from the Bacillus Genetic Stock Center (BGSC 4BD1). Initial production of the sactipeptide was assessed in BHI (BrainHeart Infusion from Beckton Dickinson), LB, and TGY (5 g/L tryptone, $1 \mathrm{~g} / \mathrm{L}$ glucose, and $3 \mathrm{~g} / \mathrm{L}$ yeast extract), and a mass consistent with huazacin was observed by MALDI-TOF-MS with all three media (Figure S2). For production, bacteria were grown in LB at $37{ }^{\circ} \mathrm{C}$ with shaking at $200 \mathrm{rpm}$ for $30 \mathrm{~h}$. Cells were harvested at $4,000 \times \mathrm{g}$ for $15 \mathrm{~min}$ and the harvested cells were extracted with $35 \mathrm{~mL} \mathrm{MeOH} / \mathrm{L}$ culture for $3 \mathrm{~h}$. The extracts were then clarified by centrifugation at $4,000 \times \mathrm{g}$ for $10 \mathrm{~min}$. Clarified extract was then dried on $10 \mathrm{~g}$ Celite 545 Filter Aid (Fisher) by rotary evaporation at $25^{\circ} \mathrm{C}$.

To purify huazacin, the dried extract was subjected to chromatographic separation using a CombiFlash EZ Prep MPLC fitted with a TeleDyne Isco $43 \mathrm{~g} \mathrm{C} 18$ cartridge under the following conditions: solvent A $\left(10 \mathrm{mM} \mathrm{NH}_{4} \mathrm{HCO}_{3}\right)$, solvent $\mathrm{B}(\mathrm{MeCN})$ and the following linear gradient combinations: 4 Column Volumes (CV) $5 \%$ B, 1 CV 50\% B, 6 CV 100\% B, 4 CV 100\% B. Fractions containing huazacin (as assessed by MALDI-TOF-MS) were dried by rotary evaporation at $25{ }^{\circ} \mathrm{C}$. After resuspension in $20 \% \mathrm{MeOH} / 80 \%$ $10 \mathrm{mM} \mathrm{NH}_{4} \mathrm{HCO}_{3}$ and clarified by centrifugation $(17,000 \times \mathrm{g}, 10 \mathrm{~min})$, semi-pure huazacin was further purified using an Agilent 1200 HPLC system fitted with a Thermo Betasil C18 $5 \mu \mathrm{m}$ particle size, $10 \times 250$ $\mathrm{mm}$ operating at $4 \mathrm{~mL} / \mathrm{min}$ under the following conditions: solvent $\mathrm{A}\left(10 \mathrm{mM} \mathrm{NH} \mathrm{HCO}_{3}\right)$, solvent $\mathrm{B}$ $(\mathrm{MeCN})$ and the following gradient: $\mathrm{t}=0 \mathrm{~min}, 20 \% \mathrm{~B}, \mathrm{t}=3 \mathrm{~min}, 20 \% \mathrm{~B}, \mathrm{t}=13 \mathrm{~min}, 40 \% \mathrm{~B}, \mathrm{t}=16 \mathrm{~min} 95 \% \mathrm{~B}$, $\mathrm{t}=21 \mathrm{~min} 95 \% \mathrm{~B}, \mathrm{t}=24 \mathrm{~min}, 20 \% \mathrm{~B}, \mathrm{t}=29 \mathrm{~min}, 20 \% \mathrm{~B}$. The column was equilibrated at starting conditions for $10 \mathrm{~min}$ before each injection. Fractions containing huazacin (by MALDI-TOF-MS) were dried by rotary evaporation at $25{ }^{\circ} \mathrm{C}$ to dryness, resuspended in $20 \% \mathrm{MeOH} / 80 \% 10 \mathrm{mM} \mathrm{NH} \mathrm{NHCO}_{3}$ and clarified by centrifugation $(17,000 \times \mathrm{g}, 10 \mathrm{~min})$. Huazacin was then purified using an Agilent $1200 \mathrm{HPLC}$ system fitted with a Thermo Betasil $\mathrm{C} 185 \mu \mathrm{m}$ particle size, $4.6 \times 250 \mathrm{~mm}$ operating at $1 \mathrm{~mL} / \mathrm{min}$ under the following conditions: solvent $\mathrm{A}\left(10 \mathrm{mM} \mathrm{NH} \mathrm{HCO}_{3}\right)$, solvent $\mathrm{B}(\mathrm{MeCN})$ and the following gradient: $\mathrm{t}=0 \mathrm{~min}, 20 \% \mathrm{~B}$, $\mathrm{t}=5 \min , 20 \% \mathrm{~B}, \mathrm{t}=8 \min , 32 \% \mathrm{~B}, \mathrm{t}=18 \min 42 \% \mathrm{~B}, \mathrm{t}=21 \min 100 \% \mathrm{~B}, \mathrm{t}=26 \min , 100 \% \mathrm{~B}, \mathrm{t}=29 \min , 20 \%$ B. The column was equilibrated at starting conditions for $10 \mathrm{~min}$ before each injection. Pure huazacin was dried under rotary evaporation at $25{ }^{\circ} \mathrm{C}$ and then lyophilized to dryness. Mass of peptide was determined using a 5-digit analytical scale and indicated a final post-purification yield of $\sim 100 \mu \mathrm{g} / \mathrm{L}$ of culture.

MALDI-TOF-MS. Matrix-assisted laser desorption/ionization time of flight mass spectrometry (MALDITOF-MS) was used to identify peptide products based upon their characteristic mass changes upon posttranslational modification, protease digestion, or chemical modification. The huazacin peptides were detected using $20 \mathrm{mg} / \mathrm{mL}$ sinapic acid in $75 \% \mathrm{MeCN}$ with $0.1 \%$ formic acid. The samples were analyzed using a Bruker Daltonics UltrafleXtreme MALDI-TOF mass spectrometer in reflector/positive mode. The freyrasin precursor peptide and fragments gave the most intense peaks when mixed with a matrix consisting 
of $20 \mathrm{mg} / \mathrm{mL}$ of $\alpha$-cyano-4-hydroxycinnamic acid and $10 \mathrm{mg} / \mathrm{mL}$ 2,5-dihydroxybenzoic acid in $60 \% \mathrm{MeCN}$ with $0.1 \%$ formic acid.

HR-ESI-MS/MS analysis. Samples for high-resolution electrospray ionization (HR-ESI) MS were first HPLC-purified or desalted by ZipTip. Next, samples were diluted $1: 1$ into ESI mix $\left(80 \% \mathrm{MeCN}_{1} 19 \% \mathrm{H}_{2} \mathrm{O}\right.$, $1 \%$ formic acid). Samples were directly infused into a ThermoFisher Scientific Orbitrap Fusion ESI-MS using an Advion TriVersa Nanomate 100. The MS was calibrated and tuned with Pierce LTQ Velos ESI Positive Ion Calibration Solution (ThermoFisher). The MS was operated using the following parameters: resolution, 100,000; isolation width (MS/MS), $2 \mathrm{~m} / \mathrm{z}$; normalized collision energy (MS/MS), 35; activation q value (MS/MS), 0.4; activation time (MS/MS), $30 \mathrm{~ms}$. Fragmentation was performed using collisioninduced dissociation (CID) at 35 to $70 \%$. Data analysis was conducted using the Qualbrowser application of Xcalibur software (Thermo-Fisher Scientific).

NMR of huazacin. The sample was prepared by dissolving $1.5 \mathrm{mg}$ (HPLC-purified and lyophilized) in 250 $\mu \mathrm{L}$ of $\mathrm{CD}_{3} \mathrm{OH}$ (Acros Organics, $>99.40 \%$ atom \% D). NMR spectra were recorded on an Agilent VNMRS $750 \mathrm{MHz}$ narrow bore magnet spectrometer equipped with a $5 \mathrm{~mm}$ triple resonance $(1 \mathrm{H}-13 \mathrm{C}-15 \mathrm{~N})$ triaxial gradient probe and pulse-shaping capabilities. Samples were held at $25{ }^{\circ} \mathrm{C}$ during acquisition. Standard Varian pulse sequences were used for each of the following experiments: 1H-NMR, 1H-1H TOCSY (80 ms mixing time), and 1H-1H NOESY (400 ms mixing time). Solvent suppression by presaturation (PRESAT) was employed for $1 \mathrm{H}, 1 \mathrm{H}-1 \mathrm{H}$ NOESY and 1H-1H TOCSY. Spectra were recorded with VNMRJ 3.2A. Spectra were imported into MestReNova 8.1.1, phased and baseline corrected. Sine Bell apodization with a first point correction of 0.5 were used with a zero fill of 1024 . Resonances were referenced internally to 4,4-dimethyl-4-silapentane-1-sulfonic acid (0.00 ppm).

Microbiology assays. Aerobic bacteria were grown in BHI with shaking $(200 \mathrm{rpm})$ at $37{ }^{\circ} \mathrm{C}$. The media for Listeria was further supplemented with $5 \mathrm{mM}$ Cys. Cultures were inoculated from glycerol backstocks, grown overnight, and then diluted into fresh media and regrown to $\mathrm{OD}_{600}=0.5$. Next, cultures were diluted 1000 -fold with fresh media and used in growth-suppression assays. Anaerobic media was prepared for Clostridium by sparging the media with $\mathrm{N}_{2}$ for $15 \mathrm{~min}$ and then autoclaved for $30 \mathrm{mins}$. After autoclaving, media was again sparged with $\mathrm{N}_{2}$ for $15 \mathrm{~min}$, transferred to an anaerobic growth chamber, and allowed to equilibrate overnight. Glycerol back stocks of $C$. sporogenes were inoculated and grown overnight without shaking. Minimum inhibitory concentrations (MICs) were determined using the microbroth dilution method. Lyophilized compounds (freyrasin/huazacin) in sterile-filtered DMSO were added at a starting concentration of $256 \mu \mathrm{M}$. Two-fold serial dilutions were performed into wells containing $\sim 5.0 \times 10^{5}$ $\mathrm{CFU} / \mathrm{mL}$. Wells were examined for turbidity after overnight incubation.

Genomic DNA extraction. Paenibacillus polymyxa ATCC 842 was grown overnight in Lysogeny Broth (LB). Genomic DNA was extracted from $5 \mathrm{~mL}$ of culture using the DNeasy UltraClean Microbial Kit (MOBIO) according to the manufacturer's protocol.

Cloning of freyrasin. Based on previous expression of sactipeptides in E. coli, ${ }^{13,14}$ a pETDuet-1 vector was used for co-expression of the freyrasin (SCIFF/ranthipeptide) precursor peptide, PapA, and the radical SAM enzyme (WP_019688962.1), PapB, from Paenibacillus polymyxa ATCC 842. The pETDuet vector was modified to encode a maltose-binding protein (MBP) tag with a tobacco etch virus (TEV) proteasecleavable linker at the N-terminus of the precursor peptide in the first multiple cloning site (MCS). The expression plasmid was constructed using restriction enzyme cloning (BamHI and NotI for the precursor peptide; AatII and XhoI for the radical SAM). The genes for $p a p A$ and $p a p B$ were amplified from genomic DNA using Q5 polymerase and the primers in Table S1. The amplicons were purified with a QIAquick PCR purification kit, digested with the appropriate restriction enzymes, and then purified again using the QIAquick PCR purification kit. The peptide-encoding gene was then sequentially ligated into similarly prepared pETDuet plasmid to create pET-Duet-MCS1: MBP-PapA MCS2: PapB. 
Preparation of freyrasin. BL21-RIPL (DE3) cells were transformed with pET-Duet-MCS1: MBP-PapA MCS2: PapB containing the MBP-tagged freyrasin precursor peptide (MCS1) and tagless rSAM (MCS2) and streaked onto LB agar plates supplemented with $100 \mu \mathrm{g} / \mathrm{mL}$ ampicillin and $34 \mu \mathrm{g} / \mathrm{mL}$ chloramphenicol. A single colony was used to inoculate a $10 \mathrm{~mL}$ starter culture with the same antibiotics. After $5 \mathrm{~h}$ of growth at $37{ }^{\circ} \mathrm{C}, 1 \mathrm{~L}$ of culture in a flat bottom $4 \mathrm{~L}$ flask was inoculated and grown to $\sim 0.8 \mathrm{OD}_{600}$. At this density, expression was induced with $0.6 \mathrm{mM}$ isopropyl $\beta$-D-1-thiogalactopyranoside (IPTG) and the culture was shaken at $100 \mathrm{rpm}$ for $16 \mathrm{~h}$ at room temperature. The cells were harvested by centrifugation at $4,000 \times \mathrm{g}$ for $15 \mathrm{~min}$. Cell pellets were frozen at $-20{ }^{\circ} \mathrm{C}$ if not used immediately.

To purify freyrasin, the harvested cells were resuspended in ice cold lysis buffer [50 mM Tris $\mathrm{pH} 7.5,500$ $\mathrm{mM} \mathrm{NaCl}, 2.5 \%(v / v)$ glycerol, $0.1 \%(v / v)$ Triton X-100] supplemented with $3 \mathrm{mg} / \mathrm{mL}$ lysozyme. The samples were subjected to three rounds of sonication for $30 \mathrm{~s}$ with 10 min equilibration periods at $4{ }^{\circ} \mathrm{C}$ between the sonication steps. The lysate was centrifuged for $60 \mathrm{~min}$ at $17,000 \times \mathrm{g}$ to remove the cellular debris. The clarified lysate was loaded into a gravity flow column with amylose resin (pre-equilibrated in lysis buffer). The column was then washed with wash buffer [50 mM Tris $\mathrm{pH} 7.5,500 \mathrm{mM} \mathrm{NaCl}, 2.5 \%$ $(v / v)$ glycerol] and then eluted with elution buffer [50 mM Tris $\mathrm{pH} 7.5,300 \mathrm{mM} \mathrm{NaCl}, 2.5 \%(v / v)$ glycerol, $10 \mathrm{mM}$ maltose]. The eluted protein was collected in Amicon Ultra $15 \mathrm{~mL}$ centrifugal filters [30 kDa NMWL (Nominal Molecular Weight Limit)]. The protein was concentrated and a 10-fold buffer exchange was performed into storage buffer [50 mM HEPES, $300 \mathrm{mM} \mathrm{NaCl}, 2.5 \%(v / v)$ glycerol].

MBP-tagged freyrasin $\left(500 \mu \mathrm{L}\right.$ of $20 \mathrm{mg} / \mathrm{mL}$ solution) was digested with TEV protease $(80 \mu \mathrm{g})$ at $30{ }^{\circ} \mathrm{C}$ for $16 \mathrm{~h}$ to remove the MBP tag. Unexpectedly, TEV protease processed multiple sites within the freyrasin leader peptide, in addition to the intended site (Figure S17). However, one of the truncated peptides removed the entire predicted leader peptide, resulting in more ideal peptide for NMR analysis due to the reduction of amino acid multiplicity. Thus, this modified peptide was purified using an Agilent 1200 series HPLC fitted with a $10 \times 250 \mathrm{~mm}$ Betasil C18 column (Fisher Scientific). A gradient elution was used with solvent $\mathrm{A}(0.1 \%$ formic acid) and solvent $\mathrm{B}(\mathrm{MeCN}+0.1 \%$ formic acid) according to the following linear gradient combinations: at $\mathrm{t}=0 \mathrm{~min}, 10 \% \mathrm{~B} ; \mathrm{t}=2 \mathrm{~min}, 10 \% \mathrm{~B} ; \mathrm{t}=19 \min , 35.5 \% \mathrm{~B} ; \mathrm{t}=22 \mathrm{~min}, 95 \% \mathrm{~B} ; \mathrm{t}=27$ $\min , 95 \% \mathrm{~B} ; \mathrm{t}=31 \mathrm{~min}, 10 \% \mathrm{~B} ; \mathrm{t}=45 \mathrm{~min}, 1 \% \mathrm{~B}$. Fractions containing pure freyrasin were dried under rotary evaporation at $25{ }^{\circ} \mathrm{C}$ and then lyophilized to dryness. Mass of peptide was determined using a 5-digit analytical scale and indicated a final yield after purification of $\sim 250 \mu \mathrm{g} / \mathrm{L}$ of culture.

NMR of freyrasin. The sample was prepared by dissolving $1.7 \mathrm{mg}$ (HPLC-purified and lyophilized) in 350 $\mu \mathrm{L}$ of 9:1 $\mathrm{H}_{2} \mathrm{O}: \mathrm{D}_{2} \mathrm{O}$ (99.96 atom \% D; Sigma-Aldrich) supplemented with $3 \mu \mathrm{L}$ of $1 \mathrm{M} \mathrm{NaOH}$ (aq). This sample was then neutralized with $4 \mu \mathrm{L}$ of $1 \mathrm{M} \mathrm{HCl}(\mathrm{aq})$. The sample was then sonicated at room temperature for $15 \mathrm{~min}$. NMR spectra were recorded on an Agilent VNMRS $750 \mathrm{MHz}$ narrow bore magnet spectrometer equipped with a $5 \mathrm{~mm}$ triple resonance $(1 \mathrm{H}-13 \mathrm{C}-15 \mathrm{~N})$ triaxial gradient probe and pulse-shaping capabilities. Samples were held at $25{ }^{\circ} \mathrm{C}$ during acquisition. Standard Varian pulse sequences were used for each of the following experiments: $1 \mathrm{H}, 1 \mathrm{H}-1 \mathrm{H}$ TOCSY ( $80 \mathrm{~ms}$ mixing time), and 1H-1H NOESY (400 ms mixing time). Solvent suppression by presaturation (PRESAT) was employed for $1 \mathrm{H}$ and $1 \mathrm{H}-1 \mathrm{H}$ NOESY experiments, while $1 \mathrm{H}-1 \mathrm{H}$ TOCSY spectra were recorded using a $\mathrm{z}$-filter and $\mathrm{H} 2 \mathrm{O}$ flipback pulse. 1:9 $\mathrm{D}_{2} \mathrm{O}: \mathrm{H}_{2} \mathrm{O}$ was used as the solvent. Spectra were recorded with VNMRJ 3.2A. Spectra were imported into MestReNova 8.1.1, phased and baseline corrected. Sine Bell II apodization with a first point correction of 0.5 were used with a zero fill of 1024 . Resonances were referenced internally to the solvent peak (4.64 ppm, HOD).

Quantum mechanical system preparation and calculations. Two tripeptides, one being Gly-Cys-Gly and the other being Gly-Asp-Gly, were assembled using the Model Kit Peptide builder of Spartan 16 version 2.0.7 (Wavefunction, Inc. Irvine, CA, http://www.wavefun.com). The N-terminus of both peptides were capped as $N$-acetyl with the $\mathrm{C}$-terminus being capped as the $O$-methyl ester to mitigate any charge effects. 
The initial peptide backbone dihedral angles were set as $\psi=180^{\circ}$ and $\varphi=-180^{\circ}$. The tripeptides were further modified in Molecular Operating Environment MOE 2016.08 (Chemical Computing Group ULC) to match structures to model the natural product structures reported in this study, in which the Cys $\mathrm{S}$ atom is bonded either to the alpha- or beta-carbon of the recipient Asp residue, termed S-C $\alpha$ and S-C $\beta$, respectively (Figure S16). Finally, the two thioether-crosslinked peptides were imported to Avogadro 1.2.0, http://avogadro.cc), ${ }^{15}$ where the structures were subjected to a brief classical energy minimization with the steepest descent algorithm and the UFF force field, ${ }^{16}$ and input files for quantum calculations were prepared. Quantum mechanical geometry optimization followed by electronic and zero-point energy calculations were performed using Gaussian 09, ${ }^{17}$ with density functional theory (DFT) methods using the B3LYP hybrid functional and 6-31G(d) basis sets. ${ }^{18,19}$

Cloning of thermocellin. E. coli optimized genes encoding for the thermocellin precursor peptide (cteA) and cognate rSAM (cteB) were ordered from Synbio Technologies (Monmouth Junction, NJ, USA) using sequences previously described by Grove et al. ${ }^{3}$ Similar to coexpression of freyrasin described above, cteA was inserted downstream of a MBP affinity tag to yield a construct for both overexpression of unmodified precursor as well as feedstock for subsequent insertion of tagless $c t e B$ in the second MCS of the pET-Duet vector via Gibson assembly. Additionally, for overexpressions involving thermocellin, the AmpR resistance marker was swapped with KanR.

Overexpression of unmodified, MBP-tagged thermocellin precursor CteA. $p E T-D u e t-M C S 1$ : MBPCteA was transformed into BL21(DE3) E. coli and plated on LB media containing $50 \mu \mathrm{g} / \mathrm{mL}$ kanamycin. A single colony was used to inoculate $10 \mathrm{~mL}$ of LB liquid media containing $50 \mu \mathrm{g} / \mathrm{mL}$ kanamycin as an overnight starter culture. This culture was then used to inoculate $1 \mathrm{~L}$ of LB liquid media containing 50 $\mu \mathrm{g} / \mathrm{mL}$. The culture was allowed to grow at $37^{\circ} \mathrm{C}$ with $250 \mathrm{rpm}$ shaking until reaching an $\mathrm{OD}_{600}$ of 1.0. Upon reaching density, the culture was cooled in an ice bath for $15 \mathrm{~min}$ prior to the addition of $100 \mathrm{mg} / \mathrm{L}$ $\mathrm{FeSO}_{4}$ and IPTG to a final concentration of $0.4 \mathrm{mM}$. Induction was carried out for $16 \mathrm{~h}$ at $22{ }^{\circ} \mathrm{C}$ with shaking at $250 \mathrm{rpm}$.

Isotopic labeling of CteA with $\mathbf{L}-\left[4-{ }^{13} \mathbf{C}, 2,3-{ }^{2} \mathbf{H}_{2}\right]-\mathbf{T h r}$. L-[4- $\left.{ }^{13} \mathrm{C}, 2,3-{ }^{2} \mathrm{H}_{2}\right]-\mathrm{Thr}$ was purchased from Cambridge Isotope Laboratories (Tewksbury, MA, USA). pET-Duet-MCS1: MBP-CteA was transformed into RF2 E. coli, a threonine auxotroph owing a deletion of threonine synthetase $(t h r C)$, and plated on LB contianing $50 \mu \mathrm{g} / \mathrm{mL}$ kanamycin. A single colony was used to inoculate $5 \mathrm{~mL}$ of LB supplemented with 50 $50 \mu \mathrm{g} / \mathrm{mL}$ kanamycin to create an overnight culture which was subsequently used to inoculate M9 media (22 $\mathrm{mM} \mathrm{KH}_{2} \mathrm{PO}_{4}, 43 \mathrm{mM} \mathrm{Na}_{2} \mathrm{HPO}_{4}, 8.5 \mathrm{mM} \mathrm{NaCl}, 9 \mathrm{mM}\left(\mathrm{NH}_{4}\right)_{2} \mathrm{SO}_{4}, 22.2 \mathrm{mM}$ glucose) supplemented with $50 \mu \mathrm{g} / \mathrm{mL}$ kanamycin at a ratio of 1:100. Additionally, $1 \mathrm{mM} \mathrm{L}-\left[4-{ }^{13} \mathrm{C}, 2,3-{ }^{2} \mathrm{H}_{2}\right]-\mathrm{Thr}$ and $2 \mathrm{mM}$ L-Gly and L-Ile were added, the latter two to suppress isotopic scrambling by Thr-catabolizing pathways. ${ }^{20}$ Overexpression was otherwise carried out identically for natural-abundance MBP-tagged CteA, described immediately above.

Coexpression of CteA with cognate rSAM CteB. pET-Duet-MCS1: MBP-CteA-MCS2: CteB was transformed into E. coli RF2 cells harboring the pSUF accessory plasmid (chloramphenicol marked), which overexpresses the suf operon from E. coli and aids in Fe-S cluster formation, ${ }^{21}$ and plated on LB supplemented with $50 \mu \mathrm{g} / \mathrm{mL}$ kanamycin. A single colony was used to inoculate a starter culture of $5 \mathrm{~mL}$ LB containing $50 \mu \mathrm{g} / \mathrm{mL}$ kanamycin and $34 \mu \mathrm{g} / \mathrm{mL}$ chloramphenicol. This overnight culture was used to coexpress $\mathrm{Cte} \mathrm{A}$ and $\mathrm{CteB}$ using the same procedure described above for isotopically labeled CteA with the exception that shaking was done at $75 \mathrm{rpm}$ to produce quasi-anaerobic conditions and prevent dissolved oxygen from poisoning Fe-S clusters. The threonine used was either L- $\left[4-{ }^{13} \mathrm{C}, 2,3-{ }^{2} \mathrm{H}_{2}\right]-\mathrm{Thr}$ (for labeled CteA) or L-Thr for natural abundance CteA.

Purification of MBP-tagged CteA. For all variants of CteA expression (modified, unmodified, labeled, and natural abundance), MBP-tagged CteA was purified by amylose resin as described above for freyrasin. 
$\mathrm{N}$-ethylmaleimide labeling of CteA. To assess the state of modification of CteA in coexpression assays, obviate convolution caused by disulfide formation, and facilitate separation of modified and unmodified peptide, unmodified Cys groups were labeled with $N$-ethylmaleimide (NEM) prior to HPLC and MS/MS analysis. Purified MBP-tagged CteA was cleaved with TEV protease at a ratio of $1: 10$ for $2 \mathrm{~h}$ at $25^{\circ} \mathrm{C}$. Free MBP and TEV protease were then removed by reverse Ni-NTA chromatography as previously described. ${ }^{22}$ The cleaved peptide was then reacted with a 10-fold molar excess of TCEP (relative to concentration of free thiol groups) at $37{ }^{\circ} \mathrm{C}$ for $10 \mathrm{~min}$. A 10 -fold molar excess of NEM was added an incubated at $37^{\circ} \mathrm{C}$ for 30 min. Lastly, a 15-fold molar excess of DTT was added to quench the reaction.

Solid-phase extraction of NEM-labeled CteA. A 500 g C18 solid-phase extraction (SPE) cartridge (ThermoFisher) was equilibrated with 10 column volumes (CV) of $\mathrm{MeCN}$ followed by $10 \mathrm{CV}$ of $0.1 \%$ formic acid prior to applying the bulk CteA NEM-labeling mixture. Salts were removed with $5 \mathrm{CV}$ of $0.1 \%$ formic acid prior to elution with $80 \% \mathrm{MeCN}+0.1 \%$ formic acid. The elution was then lyophilized to dryness prior to treatment with GluC endoproteinase.

GluC endoproteinase treatment of CteA. After SPE, the lyophilized peptide was resuspended in protein storage buffer (composition above) and treated with GluC endoproteinase (Worthingon Biochemical Corporation, Lakewood, NJ, USA) at a ratio of 1:20 (by mass) prior to HPLC and subsequent MS/MS analysis.

HPLC purification and MS/MS analysis of GluC-treated, NEM-labeled CteA. GluC-treated CteA was injected onto a $250 \times 4.6 \mathrm{~mm}$ Nucleodur C18 Gravity $5 \mu \mathrm{m}$ column (Machery-Nagel, Düren, Germany) pre-equilibrated with $95 \% \mathrm{~A} / 5 \% \mathrm{~B}(\mathrm{~A}=0.1 \%$ formic acid; $\mathrm{B}=\mathrm{MeCN}+0.1 \%$ formic acid). Peptide fragments were then subjected to the following HPLC gradient: 5 min at 5\% B prior to a gradient reaching $95 \%$ B over 45 min followed by a hold at $5 \mathrm{~min}$ at $95 \%$ B. Fractions were collected by monitoring the absorbance at $220 \mathrm{~nm}$ and analyzed by MALDI-TOF-MS. Fractions containing NEM-labeled core peptides of interest were lyophilized to dryness, reconstituted in $80 \% \mathrm{MeCN}$ with $1 \%$ acetic acid and directly infused into a ThermoFisher Scientific Orbitrap Fusion ESI-MS using an Advion TriVersa Nanomate 100. MS/MS analysis was carried out as described above for huazain and freyrasin. 
Table S1: Primers used in this study. Restriction sites are highlighted in red.

\begin{tabular}{|l|l|}
\hline \multicolumn{1}{|c|}{ Primer } & \multicolumn{1}{c|}{ Nucleotide sequence (5' to $\mathbf{3}^{\prime}$ ) } \\
\hline MBP Fwd (for sequencing) & ATGAAGCCCTGAAAGACG \\
\hline PapA_BamHI_Fwd & AAAGGATCCCATGTTGAAGCAAATCAATGTTATTGCAGG \\
\hline PapA_Notl_Rev & AAAGCGGCCGCCTAGGTATCCGATTTGATACAAAAATCACTTGC \\
\hline PapB_AatlI_Fwd & AAAGACGTCATGGCTAATCTGATTCAGGATAGAGAAGATG \\
\hline PapB_Xhol_Rev & AAACTCGAGCTAACCAAAAAGGATGCTTCTTTGTTCC \\
\hline CteA_BamHI_Fwd & AAGGATCCAATGAAACACATCAAAATCCTGAACGGC \\
\hline CteA_Notl_Rev & AAGCGGCCGCTTAACGTTTTTCGCAGCTCTGATTCGCAACG \\
\hline CteB_Gibson_Insert_F & GTTAAGTATAAGAAGGAGATATACATATGGCGATGATCCACAAATTCAGC \\
\hline CteB_Gibson_Insert_R & GATATCCAATTGAGATCTGCTTACATTTCCTGTGCTTTAATCCACAGC \\
\hline CteB_Gibson_Backbone_F & GCTGTGGATTAAAGCACAGGAAATGTAAGCAGATCTCAATTGGATATC \\
\hline CteB_Gibson_Backbone_R & GCTGAATTTGTGGATCATCGCCATATGTATATCTCCTTCTTATACTTAAC \\
\hline
\end{tabular}


Table S2. Most common Pfams that co-occur within 8 open-reading frames of the rSAM. Listed are Pfams that co-occur with a frequency greater than 5\%.

\begin{tabular}{|c|c|c|}
\hline PFAM & Description & Count \\
\hline PF04055 & Radical_SAM Radical SAM superfamily & 4814 \\
\hline PF13353 & Fer4_12 4Fe-4S single cluster domain & 3871 \\
\hline PF00005 & $\mathrm{ABC}$ _tran $\mathrm{ABC}$ transporter & 2370 \\
\hline PF13165 & DUF4001 Protein of unknown function (DUF4001) (SCIFF precursor peptide) & 1703 \\
\hline PF13304 & AAA_21 AAA domain putative AbiEii toxin Type IV TA system & 1234 \\
\hline PF02355 & SecD_SecF Protein export membrane protein & 942 \\
\hline PF00664 & ABC_membrane $\mathrm{ABC}$ transporter transmembrane region & 931 \\
\hline PF00072 & Response_reg Response regulator receiver domain & 676 \\
\hline PF12670 & DUF3792 Protein of unknown function (DUF3792) & 650 \\
\hline PF03176 & MMPL MMPL family & 650 \\
\hline PF00082 & Peptidase_S8 Subtilase family & 638 \\
\hline PF00753 & Lactamase_B Metallo-beta-lactamase superfamily & 629 \\
\hline PF12706 & Lactamase_B_2 Beta-lactamase superfamily domain & 593 \\
\hline PF01368 & DHH DHH family & 592 \\
\hline PF12833 & HTH_18 Helix-turn-helix domain & 582 \\
\hline PF02518 & HATPase_c Histidine kinase- DNA gyrase B- and HSP90-like ATPase & 572 \\
\hline PF00156 & Pribosyltran Phosphoribosyl transferase domain & 568 \\
\hline PF02699 & YajC Preprotein translocase subunit & 561 \\
\hline PF08992 & QH-AmDH_gamma Quinohemoprotein amine dehydrogenase gamma subunit & 538 \\
\hline PF09100 & Qn_am_d_aIV Quinohemoprotein amine dehydrogenase alpha subunit domain IV & 530 \\
\hline PF02272 & DHHA1 DHHA1 domain & 524 \\
\hline PF04607 & RelA_SpoT Region found in RelA / SpoT proteins & 524 \\
\hline PF04820 & Trp_halogenase Tryptophan halogenase & 522 \\
\hline PF13328 & HD_4 HD domain & 520 \\
\hline PF01702 & TGT Queuine tRNA-ribosyltransferase & 518 \\
\hline PF09098 & Dehyd-heme_bind Quinohemoprotein amine dehydrogenase alpha subunit haem binding & 506 \\
\hline PF00528 & BPD_transp_1 Binding-protein-dependent transport system inner membrane component & 506 \\
\hline PF02363 & C_tripleX Cysteine rich repeat & 458 \\
\hline PF07690 & MFS_1 Major Facilitator Superfamily & 434 \\
\hline PF14681 & UPRTase Uracil phosphoribosyltransferase & 422 \\
\hline PF00152 & tRNA-synt_2 tRNA synthetases class II (D K and N) & 416 \\
\hline PF00171 & Aldedh Aldehyde dehydrogenase family & 409 \\
\hline PF13393 & tRNA-synt_His Histidyl-tRNA synthetase & 363 \\
\hline PF02547 & Queuosine_synth Queuosine biosynthesis protein & 362 \\
\hline PF00392 & GntR Bacterial regulatory proteins gntR family & 350 \\
\hline PF00587 & tRNA-synt_2b tRNA synthetase class II core domain $(\mathrm{G} \mathrm{H} \mathrm{P} \mathrm{S} \mathrm{and} \mathrm{T)}$ & 348 \\
\hline PF02580 & Tyr_Deacylase D-Tyr-tRNA(Tyr) deacylase & 347 \\
\hline PF12669 & P12 Virus attachment protein $\mathrm{p} 12$ family & 345 \\
\hline PF02938 & GAD GAD domain & 344 \\
\hline
\end{tabular}




\begin{tabular}{|l|l|l|}
\hline PF07549 & Sec_GG SecD/SecF GG Motif & 333 \\
\hline PF00106 & adh_short short chain dehydrogenase & 330 \\
\hline PF05491 & RuvB_C Holliday junction DNA helicase ruvB C-terminus & 329 \\
\hline PF05496 & RuvB_N Holliday junction DNA helicase ruvB N-terminus & 326 \\
\hline PF01330 & RuvA_N RuvA N terminal domain & 325 \\
\hline PF01965 & DJ-1_PfpI DJ-1/PfpI family & 324 \\
\hline PF07992 & Pyr_redox_2 Pyridine nucleotide-disulphide oxidoreductase & 317 \\
\hline PF00486 & Trans_reg_C Transcriptional regulatory protein C terminal & 317 \\
\hline PF00155 & Aminotran_1_2 Aminotransferase class I and II & 316 \\
\hline PF12730 & ABC2_membrane_4 ABC-2 family transporter protein & 314 \\
\hline PF14520 & HHH_5 Helix-hairpin-helix domain & 313 \\
\hline PF13561 & adh_short_C2 Enoyl-(Acyl carrier protein) reductase & 310 \\
\hline PF01381 & HTH_3 Helix-turn-helix & 302 \\
\hline PF13186 & SPASM Iron-sulfur cluster-binding domain & 296 \\
\hline PF02687 & FtsX FtsX-like permease family & 287 \\
\hline PF00990 & GGDEF Diguanylate cyclase GGDEF domain & 265 \\
\hline PF12902 & Ferritin-like Ferritin-like & 263 \\
\hline PF01276 & OKR_DC_1 Orn/Lys/Arg decarboxylase major domain & 257 \\
\hline PF05193 & Peptidase_M16_C Peptidase M16 inactive domain & 255 \\
\hline PF00501 & AMP-binding AMP-binding enzyme & 254 \\
\hline PF00158 & Sigma54_activat Sigma-54 interaction domain & 251 \\
\hline PF00702 & Hydrolase haloacid dehalogenase-like hydrolase & 246 \\
\hline PF00512 & HisKA His Kinase A (phospho-acceptor) domain & 246 \\
\hline PF00132 & Hexapep Bacterial transferase hexapeptide (six repeats) & 239 \\
\hline PF14532 & Sigma54_activ_2 Sigma-54 interaction domain & 234 \\
\hline
\end{tabular}


Table S3: ${ }^{1} \mathrm{H}$ Chemical shift values of assigned huazacin residues. Resonances are labeled based on their position within the indicated amino acid, i.e. $\mathrm{H}-\mathrm{C}_{\alpha}$ denotes the alpha carbon proton. Unassigned residues are denoted by “*” and bolded ppm values indicate aromatic proton resonances (for Tyr1, Trp2, Tyr34). The Trp indole proton is denoted by " $\mathrm{H}-\mathrm{N}_{\text {ind." }}$ "

\begin{tabular}{|c|c|c|c|c|c|c|c|c|}
\hline Residue & $\mathrm{NH}$ & $\mathrm{H}-\mathrm{C}_{\alpha}$ & $\mathrm{H}-\mathrm{C}_{\beta}$ & $\mathrm{H}-\mathrm{C}_{\gamma}$ & $\mathrm{H}-\mathrm{C}_{\delta}$ & H-C & $\mathrm{H}-\mathrm{C}_{\zeta}$ & $\mathrm{H}-\mathrm{N}_{\text {ind }}$ \\
\hline $\mathrm{Tyr}_{1}$ & - & * & $*$ & 6.93 & 6.59 & & & \\
\hline $\operatorname{Trp}_{2}$ & * & $*$ & $*$ & 7.36 & 7.27 & 7.02 & 6.91 & 10.6 \\
\hline $\mathrm{Gly}_{3}$ & 8.54 & $3.86,3.52$ & & & & & & \\
\hline $\mathrm{Cys}_{4}$ & 7.47 & 3.82 & $3.71,3.66$ & & & & & \\
\hline $\mathrm{Ala}_{5}$ & 7.99 & 3.97 & 1.51 & & & & & \\
\hline $\mathrm{Leu}_{6}$ & 7.64 & 3.94 & 1.64 & 1.45 & $0.84,0.80$ & & & \\
\hline $\mathrm{Lys}_{7}$ & 7.11 & 3.92 & 1.72 & 1.32 & 1.55 & 2.74 & & \\
\hline $\mathrm{Cys}_{8}$ & 8.3 & 3.71 & $3.17,2.83$ & & & & & \\
\hline Ala $_{9}$ & 7.21 & 3.89 & 1.42 & & & & & \\
\hline Gly $_{10}$ & 8.57 & $3.85,3.7$ & & & & & & \\
\hline Pro $_{11}$ & - & 4.11 & $1.79,1.75$ & 1.53 & $2.24,2.17$ & & & \\
\hline Cys $_{12}$ & 7.99 & 3.79 & $3.61,3.16$ & & & & & \\
\hline $\mathrm{Leu}_{13}$ & 8.24 & 3.73 & $1.78,1.69$ & 1.57 & 0.79 & & & \\
\hline Gly $_{14}$ & 8.2 & $3.62,3.55$ & & & & & & \\
\hline $\mathrm{Val}_{15}$ & 7.37 & 3.76 & 2.02 & $1.02,0.83$ & & & & \\
\hline $\mathrm{Cys}_{16}$ & 8.84 & 3.8 & 3.6 & 2.79 & & & & \\
\hline $\mathrm{Ala}_{17}$ & 7.9 & 3.92 & 1.34 & & & & & \\
\hline $\mathrm{Ile}_{18}$ & 7.49 & 3.66 & 1.96 & $1.83,1.19,0.92$ & 0.85 & & & \\
\hline $\mathrm{Asp}_{19}$ & 7 & 4.9 & $2.77,2.51$ & & & & & \\
\hline $\mathrm{Thr}_{20}$ & 8.24 & 4.29 & 3.97 & 1.25 & & & & \\
\hline $\mathrm{Ala}_{21}$ & 8.47 & 4.39 & 1.41 & & & & & \\
\hline $\operatorname{Ser}_{22}$ & 7.4 & 4.99 & 3.8 & & & & & \\
\hline $\mathrm{PrO}_{23}$ & - & 4.94 & $2.02,1.96$ & 1.8 & $3.85,3.80$ & & & \\
\hline $\mathrm{Val}_{24}$ & 7.85 & 3.5 & 1.95 & $0.93,0.87$ & & & & \\
\hline $\mathrm{Met}_{25}$ & 7.83 & 4.4 & $2.31,2.25$ & 2.62 & - & 1.94 & & \\
\hline $\mathrm{Asp}_{26}$ & $*$ & $*$ & $*$ & & & & & \\
\hline $\mathrm{Ala}_{27}$ & 7.24 & 3.79 & 1.51 & & & & & \\
\hline $\mathrm{Val}_{28}$ & 7.92 & 3.64 & 2.16 & $1.05,0.93$ & & & & \\
\hline $\mathrm{Gly}_{29}$ & 9.59 & $4.12,3.58$ & & & & & & \\
\hline $\mathrm{Thr}_{30}$ & 8.79 & - & 4.45 & 1.11 & & & & \\
\hline $\mathrm{Ala}_{31}$ & 7.89 & 3.89 & 1.48 & & & & & \\
\hline $\mathrm{Ser}_{32}$ & 8.62 & 3.77 & 3.58 & & & & & \\
\hline Gly $_{33}$ & 7.53 & 3.84 & 3.69 & & & & & \\
\hline $\mathrm{Tyr}_{34}$ & 9.57 & - & $*$ & 6.57 & 6.82 & & & \\
\hline $\mathrm{Ala}_{35}$ & $*$ & 4.12 & 1.53 & & & & & \\
\hline Gly $_{36}$ & 9.5 & $4.13,3.67$ & & & & & & \\
\hline Gly $_{37}$ & 7.97 & $4.11,3.94$ & & & & & & \\
\hline $\mathrm{His}_{38}$ & 9.04 & - & $*$ & $*$ & & & & \\
\hline Gly $_{39}$ & $7 . .27$ & $3.94,3.47$ & & & & & & \\
\hline
\end{tabular}


Table S4: Antibiotic activity of freyrasin and huazacin. A small panel of Firmicutes (Gram-positive) and $\gamma$-Proteobacteria (Gram-negative; E. coli and P. aeruginosa) were evaluated for susceptibility to the two new compounds from this study $(n=1)$. Only huazacin displayed notable antibiotic activity.

\begin{tabular}{|l|c|c|}
\hline Organism & Freyrasin $(\boldsymbol{\mu M})$ & Huazacin $(\boldsymbol{\mu M})$ \\
\hline Bacillus subtilis str. 168 & 128 & 128 \\
\hline Bacillus anthracis str. Sterne & 128 & 128 \\
\hline Clostridium sporogenes ATCC 19404 & 32 & 32 \\
\hline Clostridium sporogenes ARS41091 & 64 & 64 \\
\hline Clostridium sporogenes ATCC 155761 & 32 & 32 \\
\hline Listeria monocytogenes 4b F2365 & 128 & 4 \\
\hline Staphylococcus aureus USA300 & 128 & 128 \\
\hline Escherichia coli DH5a & 128 & 128 \\
\hline Pseudomonas aeruginosa PAO1 & 32 & 32 \\
\hline
\end{tabular}


Table S5: ${ }^{1} \mathrm{H}$ Chemical shift values of assigned freyrasin residues. Resonances are labeled based on their position within the indicated amino acid, i.e. $\mathrm{H}-\mathrm{C}_{\alpha}$ denotes the alpha carbon proton. Unassigned residues are denoted by “*” and bolded ppm values indicate aromatic proton resonances (for Tyr9, Phe10 and Phe25). Both Asn residues were identified based on TOCSY correlations, but no through-space correlations could be used to assign them within the linear sequence.

\begin{tabular}{|c|c|c|c|c|c|c|c|}
\hline Residue & $\mathrm{NH}$ & $\mathrm{H}-\mathrm{C}_{\alpha}$ & $\mathrm{H}-\mathrm{C}_{\beta}$ & $\mathrm{H}-\mathrm{C}_{\gamma}$ & $\mathrm{H}-\mathrm{C}_{\delta}$ & $\mathrm{H}-\mathrm{C}_{\varepsilon}$ & $\mathrm{H}-\mathrm{C}_{\zeta}$ \\
\hline Gly $_{1}$ & - & - & - & - & - & - & \\
\hline $\mathrm{Cys}_{2}$ & 8.53 & 4.35 & $2.63,2.75$ & & & & \\
\hline $\mathrm{Ser}_{3}$ & 8.68 & 4.26 & $3.75 *$ & & & & \\
\hline $\mathrm{Ala}_{4}$ & 7.88 & 4.21 & 1.22 & & & & \\
\hline $\mathrm{Asn}_{5}$ & $*$ & $*$ & $*$ & & & & \\
\hline $\mathrm{Asp}_{6}$ & 8.15 & 4.47 & 3.71 & & & & \\
\hline $\mathrm{Ala}_{7}$ & 7.98 & 4.05 & 1.08 & & & & \\
\hline $\mathrm{Cys}_{8}$ & 8.42 & 4.57 & $2.51,2.65$ & & & & \\
\hline Tyr $_{9}$ & 8.38 & 4.23 & 2.74 & - & 6.63 & 6.89 & \\
\hline Phe $_{10}$ & 7.83 & 4.41 & $2.82,2.94$ & - & 7.04 & 7.13 & 7.17 \\
\hline $\mathrm{Cys}_{11}$ & 7.86 & 4.35 & $2.80,2.69$ & & & & \\
\hline $\mathrm{Asp}_{12}$ & 8.45 & 4.37 & 3.69 & & & & \\
\hline $\mathrm{Thr}_{13}$ & 8.12 & 4.13 & 3.98 & $1.74,1.69$ & & & \\
\hline $\operatorname{Arg}_{14}$ & 7.77 & 4.23 & $1.58,1.68$ & $1.37,1.42$ & 3.02 & 7.03 & \\
\hline $\mathrm{Asp}_{15}$ & 8.31 & 4.55 & 3.55 & & & & \\
\hline $\mathrm{Asn}_{16}$ & $*$ & $*$ & $*$ & & & & \\
\hline $\mathrm{Cys}_{17}$ & - & - & - & & & & \\
\hline Lys $_{18}$ & 8.39 & 4.23 & $1.81,1.95$ & 1.68 & $*$ & 2.3 & \\
\hline $\mathrm{Ala}_{19}$ & 8.08 & 4.14 & 1.23 & & & & \\
\hline $\mathrm{Cys}_{20}$ & 8.17 & 4.3 & $2.97,2.65$ & & & & \\
\hline $\mathrm{Asp}_{21}$ & 8.58 & 4.48 & 3.75 & & & & \\
\hline $\mathrm{Ala}_{22}$ & 8.33 & 3.94 & 1.25 & & & & \\
\hline $\mathrm{Ser}_{23}$ & 7.69 & 4.27 & 3.67 & & & & \\
\hline $\mathrm{Asp}_{24}$ & 7.83 & 4.58 & 3.58 & & & & \\
\hline $\mathrm{Phe}_{25}$ & 7.92 & 4.46 & $2.93,2.78$ & - & 7.01 & 7.13 & 7.17 \\
\hline $\mathrm{Cys}_{26}$ & 8.1 & 4.42 & $2.87,2.65$ & & & & \\
\hline $\mathrm{Ile}_{27}$ & 8.22 & 3.89 & 1.73 & $1.31,1.03,0.77$ & 0.76 & & \\
\hline $\mathrm{Lys}_{28}$ & 7.99 & 4.1 & $1.95,1.76$ & 1.69 & $*$ & 2.11 & \\
\hline $\operatorname{Ser}_{29}$ & 8.31 & 4.16 & $3.78,3.73$ & & & & \\
\hline $\mathrm{Asp}_{30}$ & 8.35 & 4.66 & 3.71 & & & & \\
\hline $\mathrm{Thr}_{31}$ & 8.3 & 4.47 & 4.01 & $2.58,2.53$ & & & \\
\hline
\end{tabular}


Table S6: MS/MS Expected and observed masses of diagnostic ions.

Huazacin (spectrum shown in Figure S3)

\begin{tabular}{|l|r|r|c|}
\hline Species & $\begin{array}{r}\text { Predicted } \\
\text { mass }\end{array}$ & $\begin{array}{r}\text { Observed } \\
\text { mass }\end{array}$ & $\begin{array}{r}\text { Error } \\
\text { (ppm) }\end{array}$ \\
\hline$[\mathrm{M}+3 \mathrm{H}]^{3+}$ & 1241.53933 & 1241.53955 & 0.2 \\
\hline$[\mathrm{M}-62]^{3+}$ & 1220.87253 & 1220.87187 & 0.5 \\
\hline b2 & 350.14992 & 350.14981 & 0.3 \\
\hline b3 & 407.17138 & 407.17082 & 1.4 \\
\hline b4 & 510.18057 & 510.18023 & 0.7 \\
\hline b5 & 581.21768 & 581.21798 & 0.5 \\
\hline b6 & 694.30174 & 694.30182 & 0.1 \\
\hline b7 & 822.39671 & 822.39702 & 0.4 \\
\hline b8 & 925.40589 & 925.40619 & 0.3 \\
\hline b9 & 996.44301 & 996.44306 & 0.1 \\
\hline b10 & 1053.46447 & 1053.46446 & 0.0 \\
\hline b12 & 1253.52642 & 1253.52465 & 1.4 \\
\hline b13 & 1366.61048 & 1366.60819 & 1.7 \\
\hline b14 & 1423.63194 & 1423.63275 & 0.6 \\
\hline b15 & 1522.70036 & 1522.70195 & 1.0 \\
\hline b16 & 1625.70954 & 1625.71313 & 2.2 \\
\hline b17 & 1696.74666 & 1696.74838 & 1.0 \\
\hline b18 & 1809.83072 & 1809.83142 & 0.4 \\
\hline b19 & 1924.85766 & 1924.85798 & 0.2 \\
\hline
\end{tabular}

\begin{tabular}{|l|r|r|c|}
\hline Species & $\begin{array}{r}\text { Predicted } \\
\text { mass }\end{array}$ & $\begin{array}{r}\text { Observed } \\
\text { mass }\end{array}$ & $\begin{array}{c}\text { Error } \\
\text { (ppm) }\end{array}$ \\
\hline${\mathrm{b} 20^{2+}}^{2+}$ & 1013.45631 & 1013.45752 & 1.2 \\
\hline${\mathrm{b} 21^{2+}}^{2+}$ & 1048.97487 & 1048.97547 & 0.6 \\
\hline${\mathrm{b} 22^{2+}}^{2+}$ & 1092.99256 & 1092.99273 & 0.2 \\
\hline [b24 $^{2+62]^{2+}}$ & 1191.05315 & 1191.05521 & 1.7 \\
\hline $\mathrm{y} 5$ & 396.16261 & 396.16253 & 0.2 \\
\hline $\mathrm{y} 6$ & 557.21029 & 557.21025 & 0.1 \\
\hline $\mathrm{y} 7$ & 614.23175 & 614.23169 & 0.1 \\
\hline $\mathrm{y} 8$ & 701.26378 & 701.26471 & 1.3 \\
\hline $\mathrm{y} 9$ & 772.30089 & 772.30148 & 0.8 \\
\hline $\mathrm{y} 10$ & 871.33292 & 871.33271 & 0.2 \\
\hline $\mathrm{y} 11$ & 928.35438 & 928.35438 & 0.0 \\
\hline $\mathrm{y} 12$ & 1027.42280 & 1027.42247 & 0.3 \\
\hline $\mathrm{y} 13$ & 1098.45991 & 1098.45825 & 1.5 \\
\hline$[\mathrm{y} 14-62]$ & 1149.47082 & 1149.46942 & 1.2 \\
\hline$[\mathrm{y} 15-62]$ & 1280.51130 & 1280.51048 & 0.6 \\
\hline$[\mathrm{y} 17-62]$ & 1476.63248 & 1476.63383 & 0.9 \\
\hline$[\mathrm{y} 18-62]$ & 1563.66451 & 1563.66293 & 1.0 \\
\hline$[\mathrm{y} 21-62]$ & 1850.77624 & 1850.77531 & 0.5 \\
\hline
\end{tabular}

Freyrasin (spectrum shown in Figure S15)

\begin{tabular}{|l|r|r|c|}
\hline Species & Predicted mass & Observed mass & Error (ppm) \\
\hline$[\mathrm{M}+4 \mathrm{H}]^{4+}$ & 1397.60943 & 1397.61133 & 1.4 \\
\hline$[\mathrm{M}+5 \mathrm{H}]^{5+}$ & 1118.28900 & 1118.29030 & 1.2 \\
\hline $\mathrm{b} 27^{2+}$ & 1401.70896 & 1401.71077 & 1.3 \\
\hline $\mathrm{b} 27^{3+}$ & 934.80840 & 934.80967 & 1.4 \\
\hline $\mathrm{b} 36^{2+}$ & 1936.88826 & 1936.89112 & 1.5 \\
\hline $\mathrm{b} 36^{3+}$ & 1291.59460 & 1291.59643 & 1.4 \\
\hline $\mathrm{b} 45^{3+}$ & 1593.02409 & 1593.02567 & 1.0 \\
\hline$\left[\mathrm{b} 45-\mathrm{H}_{2} \mathrm{O}\right]^{3+}$ & 1587.02056 & 1587.02267 & 1.3 \\
\hline$[\mathrm{b} 51-\mathrm{y} 23]^{2+}$ & 1333.47910 & 1333.48131 & 1.7 \\
\hline $\mathrm{b} 45-\mathrm{y} 24$ & 1974.65098 & 1974.65379 & 1.4 \\
\hline $\mathrm{b} 51-\mathrm{y} 7$ & 692.30722 & 692.30800 & 1.1 \\
\hline $\mathrm{y} 6$ & 664.29705 & 664.29729 & 0.4 \\
\hline $\mathrm{y} 7$ & 811.36547 & 811.36650 & 1.3 \\
\hline $\mathrm{y} 16$ & 1714.65057 & 1714.65354 & 1.7 \\
\hline
\end{tabular}


L- $\left[4-{ }^{13} \mathrm{C}, 2,3-{ }^{2} \mathrm{H}_{2}\right]-$ Thr-labeled unmodified CteA (spectrum shown in Figure S22)

\begin{tabular}{|l|r|r|c|}
\hline Species & $\begin{array}{r}\text { Predicted } \\
\text { mass }\end{array}$ & $\begin{array}{r}\text { Observed } \\
\text { mass }\end{array}$ & Error (ppm) \\
\hline$[\mathrm{M}+\mathrm{H}]^{2+}$ & 1095.94439 & 1095.94163 & 2.5 \\
\hline b7 & 741.27044 & 741.27144 & 1.3 \\
\hline b8 & 812.30756 & 812.30598 & 1.9 \\
\hline b9 & 915.31674 & 915.31507 & 1.8 \\
\hline b10 & 1043.41174 & 1043.40885 & 2.8 \\
\hline b11 & 1147.47529 & 1147.47265 & 2.3 \\
\hline b12 & 1234.50732 & 1234.50495 & 1.9 \\
\hline b13 & 1337.51651 & 1337.51367 & 2.1 \\
\hline b14 & 1441.58009 & 1441.57645 & 2.5 \\
\hline b15 & 1540.64851 & 1540.64493 & 2.3 \\
\hline b16 & 1661.68562 & 1661.68172 & 2.3 \\
\hline b17 & 1725.72855 & 1725.72401 & 2.6 \\
\hline b18 & 1853.78712 & 1853.78280 & 2.3 \\
\hline b19 ${ }^{2+}$ & 970.91321 & 970.91099 & 2.3 \\
\hline b20 ${ }^{2+}$ & 1022.41781 & 1022.41581 & 2.0 \\
\hline & & & \\
\hline
\end{tabular}

\begin{tabular}{|l|r|r|c|}
\hline Species & $\begin{array}{r}\text { Predicted } \\
\text { mass }\end{array}$ & $\begin{array}{r}\text { Observed } \\
\text { mass }\end{array}$ & Error (ppm) \\
\hline y4 & 466.16022 & 466.15937 & 1.8 \\
\hline y5 & 580.20315 & 580.20212 & 1.8 \\
\hline y6 & 651.24027 & 651.23903 & 1.9 \\
\hline y7 & 750.30868 & 750.30716 & 2.0 \\
\hline y8 & 854.37227 & 854.37067 & 1.9 \\
\hline y9 & 957.38145 & 957.37931 & 2.2 \\
\hline y10 & 1044.41348 & 1044.41159 & 1.8 \\
\hline y11 & 1148.47707 & 1148.47586 & 1.1 \\
\hline y12 & 1276.57203 & 1276.57014 & 1.5 \\
\hline y15 & 1379.58121 & 1379.57763 & 2.6 \\
\hline y16 & 1450.61833 & 1450.61508 & 2.2 \\
\hline y17 & 1537.65036 & 1537.64719 & 2.1 \\
\hline y18 & 1665.70893 & 1665.70508 & 2.3 \\
\hline y19 & 1768.71812 & 1768.71454 & 2.0 \\
\hline
\end{tabular}

L-[4- $\left.{ }^{13} \mathrm{C}, 2,3-{ }^{2} \mathrm{H}_{2}\right]-\mathrm{Thr}$ and NEM-labeled, modified CteA (spectrum shown in Figure 6)

\begin{tabular}{|l|r|r|c|}
\hline Species & $\begin{array}{r}\text { Predicted } \\
\text { mass }\end{array}$ & $\begin{array}{r}\text { Observed } \\
\text { mass }\end{array}$ & Error (ppm) \\
\hline [M+H $]^{2+}$ & 1345.03247 & 1345.03170 & 0.6 \\
\hline b6 & 905.34215 & 905.34147 & 0.8 \\
\hline b7 & 991.36580 & 991.36502 & 0.8 \\
\hline b11 & 1395.55500 & 1395.55386 & 0.8 \\
\hline b12 & 1482.58703 & 1482.58546 & 1.1 \\
\hline b13 & 1710.64389 & 1710.64216 & 1.0 \\
\hline b14 & 1814.70748 & 1814.70552 & 1.1 \\
\hline b15 & 1913.77589 & 1913.77320 & 1.4 \\
\hline b16 & 1984.81300 & 1984.81037 & 1.3 \\
\hline b17 & 1049.93188 & 1049.93092 & 0.9 \\
\hline b18 & 1113.96117 & 1113.95969 & 1.3 \\
\hline b19 & 1157.47718 & 1157.47620 & 0.8 \\
\hline b20 ${ }^{2+}$ & 1271.50561 & 1271.50439 & 1.0 \\
\hline
\end{tabular}

\begin{tabular}{|l|r|r|c|}
\hline Species & $\begin{array}{r}\text { Predicted } \\
\text { mass }\end{array}$ & $\begin{array}{r}\text { Observed } \\
\text { mass }\end{array}$ & Error (ppm) \\
\hline y3 & 463.14933 & 463.14920 & 0.3 \\
\hline y4 & 591.20790 & 591.20768 & 0.4 \\
\hline y5 & 705.25083 & 705.25053 & 0.4 \\
\hline y6 & 776.28794 & 776.28771 & 0.3 \\
\hline y7 & 875.35636 & 875.35584 & 0.6 \\
\hline y8 & 979.41995 & 979.41898 & 1.0 \\
\hline y9 & 1207.47681 & 1207.47626 & 0.5 \\
\hline y10 & 1294.50884 & 1294.50876 & 0.1 \\
\hline y11 & 1627.66092 & 1627.65969 & 0.8 \\
\hline y12 & 1698.69803 & 1698.69764 & 0.2 \\
\hline y15 & 1785.73006 & 1785.72917 & 0.5 \\
\hline y16 & 957.39823 & 957.39054 & 8.0 \\
\hline y19 & 1071.42666 & 1071.42676 & 0.1 \\
\hline [y19-b15] & 1557.66045 & 1557.66016 & 0.2 \\
\hline
\end{tabular}


L- $\left[4-{ }^{13} \mathrm{C}, 2,3-{ }^{2} \mathrm{H}_{2}\right]$-Thr and NEM-labeled, unmodified CteA (spectrum shown in Figure S24)

\begin{tabular}{|l|r|r|c|}
\hline Species & $\begin{array}{r}\text { Predicted } \\
\text { mass }\end{array}$ & $\begin{array}{r}\text { Observed } \\
\text { mass }\end{array}$ & Error (ppm) \\
\hline$[\mathrm{M}+3 \mathrm{H}]^{3+}$ & 1034.11072 & 1034.10901 & 1.7 \\
\hline $\mathrm{b} 2$ & 357.12272 & 357.12257 & 0.4 \\
\hline b3 & 461.18630 & 461.18603 & 0.6 \\
\hline b4 & 548.21833 & 548.21797 & 0.7 \\
\hline b5 & 776.27520 & 776.27290 & 3.0 \\
\hline b10 & 1418.55474 & 1418.55342 & 0.9 \\
\hline b11 & 1522.61833 & 1522.61620 & 1.4 \\
\hline b12 & 1609.65036 & 1609.64826 & 1.3 \\
\hline b13 & 1837.70722 & 1837.70361 & 2.0 \\
\hline b14 & 1941.77081 & 1941.76571 & 2.6 \\
\hline b18 ${ }^{2+}$ & 1177.49283 & 1177.49097 & 1.6 \\
\hline b21 ${ }^{2+}$ & 1399.55857 & 1399.55562 & 2.1 \\
\hline y2 & 303.21392 & 303.21377 & 0.5 \\
\hline y4 & 660.31337 & 660.31282 & 0.8 \\
\hline y5 & 747.34540 & 747.34455 & 1.1 \\
\hline y6 & 875.40398 & 875.40285 & 1.3 \\
\hline
\end{tabular}

\begin{tabular}{|l|r|r|c|}
\hline Species & $\begin{array}{r}\text { Predicted } \\
\text { mass }\end{array}$ & $\begin{array}{r}\text { Observed } \\
\text { mass }\end{array}$ & Error (ppm) \\
\hline y7 & 989.44690 & 989.44584 & 1.1 \\
\hline y8 & 1060.48402 & 1060.48268 & 1.3 \\
\hline y9 & 1159.55243 & 1159.55112 & 1.1 \\
\hline y10 & 1263.61602 & 1263.61489 & 0.9 \\
\hline y11 & 1491.67288 & 1491.67100 & 1.3 \\
\hline y12 & 1578.70491 & 1578.70306 & 1.2 \\
\hline y13 & 1682.76850 & 1682.76643 & 1.2 \\
\hline y14 & 905.93564 & 905.93420 & 1.6 \\
\hline y15 & 1019.96407 & 1019.96172 & 2.3 \\
\hline y16 & 1055.48263 & 1055.48055 & 2.0 \\
\hline y17 & 1098.99865 & 1098.99674 & 1.7 \\
\hline y18 & 1163.02793 & 1163.02570 & 1.9 \\
\hline y19 & 1277.05637 & 1277.05399 & 1.9 \\
\hline y20 & 1320.57238 & 1320.56978 & 2.0 \\
\hline$y 21$ & 1372.60417 & 1372.60160 & 1.9 \\
\hline
\end{tabular}

Natural abundance Thr and NEM labeled modified CteA (spectrum shown in Figure S25)

\begin{tabular}{|l|r|r|c|}
\hline Species & $\begin{array}{r}\text { Predicted } \\
\text { mass }\end{array}$ & $\begin{array}{r}\text { Observed } \\
\text { mass }\end{array}$ & Error (ppm) \\
\hline$[\mathrm{M}+2 \mathrm{H}]^{2+}$ & 1340.50860 & 1340.50916 & 0.4 \\
\hline b3 & 458.17040 & 458.17043 & 0.1 \\
\hline b4 & 545.20242 & 545.20226 & 0.3 \\
\hline b6 & 901.31786 & 901.31698 & 1.0 \\
\hline b7 & 988.34989 & 988.35046 & 0.6 \\
\hline b8 & 1059.38701 & 1059.38622 & 0.7 \\
\hline b11 & 1389.52318 & 1389.52279 & 0.3 \\
\hline b12 & 1476.55521 & 1476.55438 & 0.6 \\
\hline b13 & 1704.61207 & 1704.61144 & 0.4 \\
\hline b14 & 1805.65975 & 1805.65848 & 0.7 \\
\hline b15 & 1904.72817 & 1904.72745 & 0.4 \\
\hline b16 & 1975.76528 & 1975.76406 & 0.6 \\
\hline b17 ${ }^{2+}$ & 1045.40802 & 1045.40767 & 0.3 \\
\hline b18 ${ }^{2+}$ & 1109.43730 & 1109.43665 & 0.6 \\
\hline b19 ${ }^{2+}$ & 1152.95332 & 1152.95293 & 0.3 \\
\hline b20 ${ }^{2+}$ & 1266.98175 & 1266.98086 & 0.7 \\
\hline
\end{tabular}

\begin{tabular}{|l|r|r|c|}
\hline Species & $\begin{array}{r}\text { Predicted } \\
\text { mass }\end{array}$ & $\begin{array}{r}\text { Observed } \\
\text { mass }\end{array}$ & Error (ppm) \\
\hline y3 & 463.14933 & 463.14931 & 0.0 \\
\hline y4 & 591.20790 & 591.20782 & 0.1 \\
\hline y5 & 705.25083 & 705.25069 & 0.2 \\
\hline y6 & 776.28794 & 776.28751 & 0.6 \\
\hline y7 & 875.35636 & 875.35602 & 0.4 \\
\hline y8 & 976.40404 & 976.40332 & 0.7 \\
\hline y9 & 1204.46090 & 1204.46063 & 0.2 \\
\hline y10 & 1291.49293 & 1291.49281 & 0.1 \\
\hline y13 & 1621.62910 & 1621.62740 & 1.0 \\
\hline y14 & 1692.66622 & 1692.66574 & 0.3 \\
\hline y15 & 1779.69825 & 1779.69807 & 0.1 \\
\hline y192+ & 1162.45061 & 1162.45016 & 0.4 \\
\hline$[y 19-b 15]$ & 1548.61273 & 1548.61283 & 0.1 \\
\hline
\end{tabular}


Table S7: Identity matrix of characterized rSAMs involved in RiPP thioether formation. The rSAM enzymes responsible for installing thioethers in SCIFF/ranthipeptides [i.e. thermocellin (CteB) and freyrasin (PapB)] are closer in overall sequence identity to QhpD than rSAM enzymes involved in sactipeptide biosynthesis [i.e. subtilosin (AlbA), sporulation killing factor (SkfB), thuricin CD ( TrnC), thurincin (ThnB), and huazacin (HuaB)]. The margin is significant (10-15\%), suggesting that the $\mathrm{SCIFF} /$ ranthipeptide rSAM enzymes may have more similar reaction chemistries to QhpD and install non$\mathrm{S}-\mathrm{C} \alpha$ thioethers. All values given are \% identity of the compared primary sequences, rounded to the nearest whole number, as determined by alignment with MAFFT $7.313 .{ }^{23} \mathrm{NCBI}$ accession used for this alignment: WP_052400378.1 (AlbA), WP_061669520.1 (SkfB), WP_098404387.1 (TrnC), EJQ15599.1 (ThnB), EEM79976.1 (HuaB), SDJ52620.1 (QhpD), WP_019688962.1 (PapB), and WP_018375754.1 (NxxcB).

\begin{tabular}{|l|c|c|c|c|c|c|c|c|c|}
\cline { 2 - 11 } \multicolumn{1}{c|}{} & AlbA & SkfB & TrnC & ThnB & HuaB & QhpD & CteB & PapB & NxxcB \\
\hline AlbA & 100 & & & & & & & & \\
\hline SkfB & 35 & 100 & & & & & & & \\
\hline TrnC & 24 & 21 & 100 & & & & & & \\
\hline ThnB & 37 & 32 & 21 & 100 & & & & & \\
\hline HuaB & 21 & 25 & 30 & 20 & 100 & & & & \\
\hline QhpD & 26 & 25 & 24 & 25 & 23 & 100 & & & \\
\hline CteB & 31 & 30 & 29 & 32 & 28 & 35 & 100 & & \\
\hline PapB & 31 & 31 & 27 & 29 & 28 & 39 & 40 & 100 & \\
\hline NxxcB & 29 & 26 & 24 & 26 & 29 & 25 & 32 & 32 & 100 \\
\hline
\end{tabular}


Figure S1: Bioinformatic analysis of sactipeptide precursor peptides. (A) Precursor peptides form distinct clusters at an alignment score of 6 and conflated at an identity score of 100\%, yielding 499 unique sequences. Characterized representatives of each grouping are shown as a diamond-shaped node. This alignment is strongly correlated with conservation of Cys residues as seen in the bottom panel. (B) Sequence logos of each cluster's core peptides. While full length precursors were used to generate these logos, only the first three residues $\mathrm{N}$-terminal of the first conserved Cys are shown. We have named the predicted group (6) "lachnocins" as the producing organisms of these compounds are predominated by the family Lachnospiraceae.

A
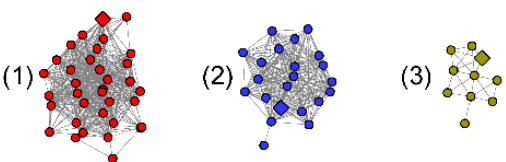

(4)

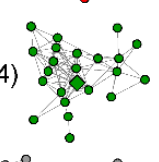

(5)

(6) $0_{0}^{0} 0$
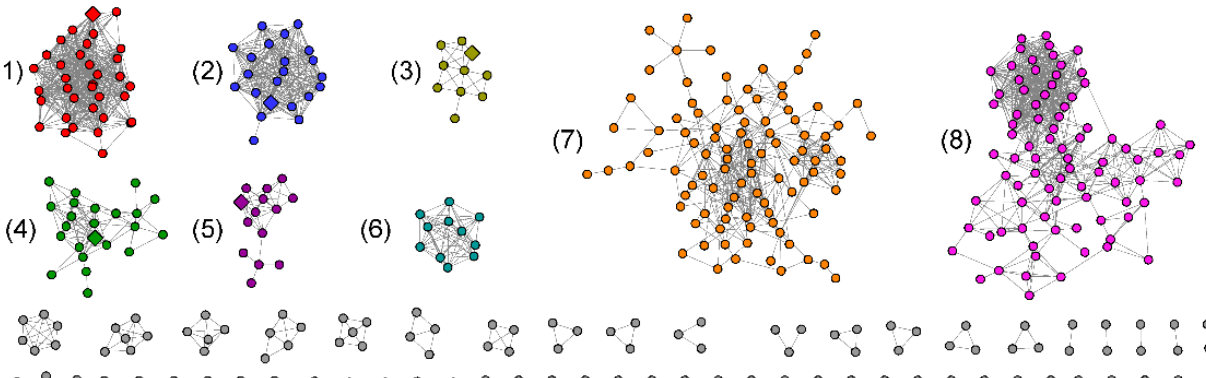

$\therefore \rightarrow 0000000$

200

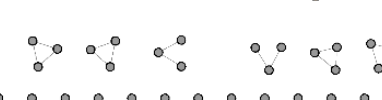

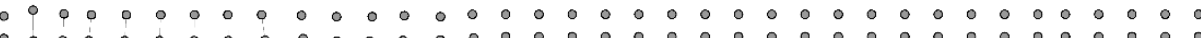

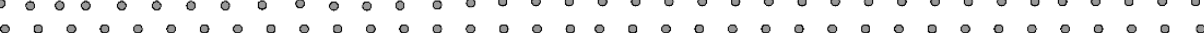

B

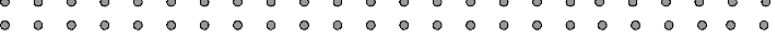

\section{(1) subtilosins and hyicins}

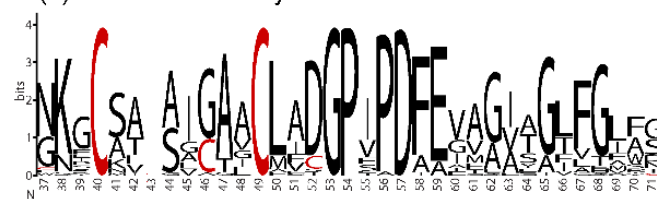

(3) thuricins

(2) sporulation killing factors
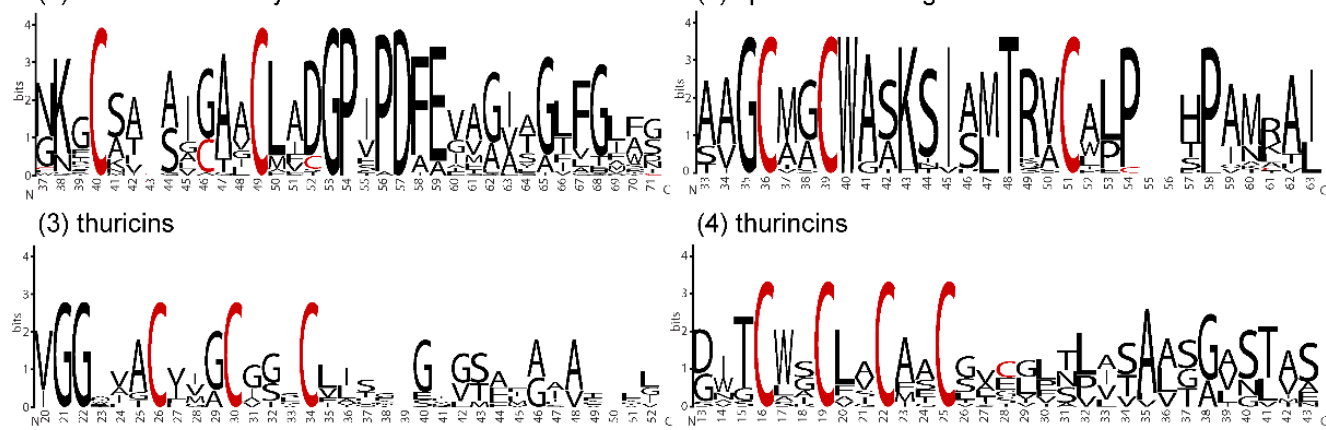

(4) thurincins

(5) huazacins [reported herein]

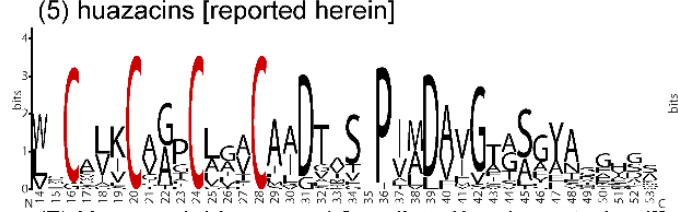

(7) Hypervariable group 1 [predicted/uncharacterized]]

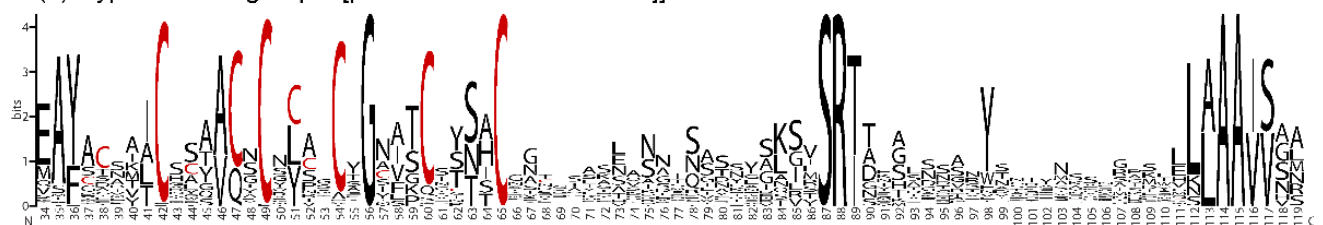

(8) Hypervariable group 2 [predicted/uncharacterized]

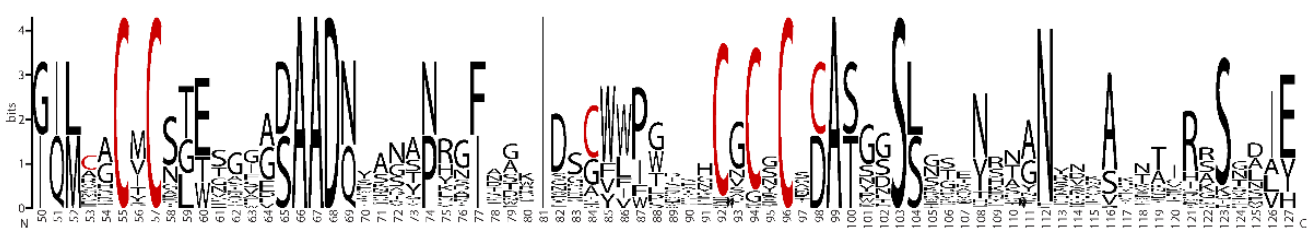


Figure S2: MALDI-TOF-MS of B. thuringiensis serovar huazhongensis extracts. (A) MALDI-TOFMS spectra of crude methanolic extractions of B. thuringiensis serovar huazhongensis grown in BHI, LB, and TGY media. High intensity masses are labeled; red indicates a mass consistent with a modified huazacin precursor peptide. (B) Masses for different $\mathrm{N}$-terminal truncations of the huazacin precursor peptide with thioether modifications (4 thioethers, one for each Cys highlighted in red).

A

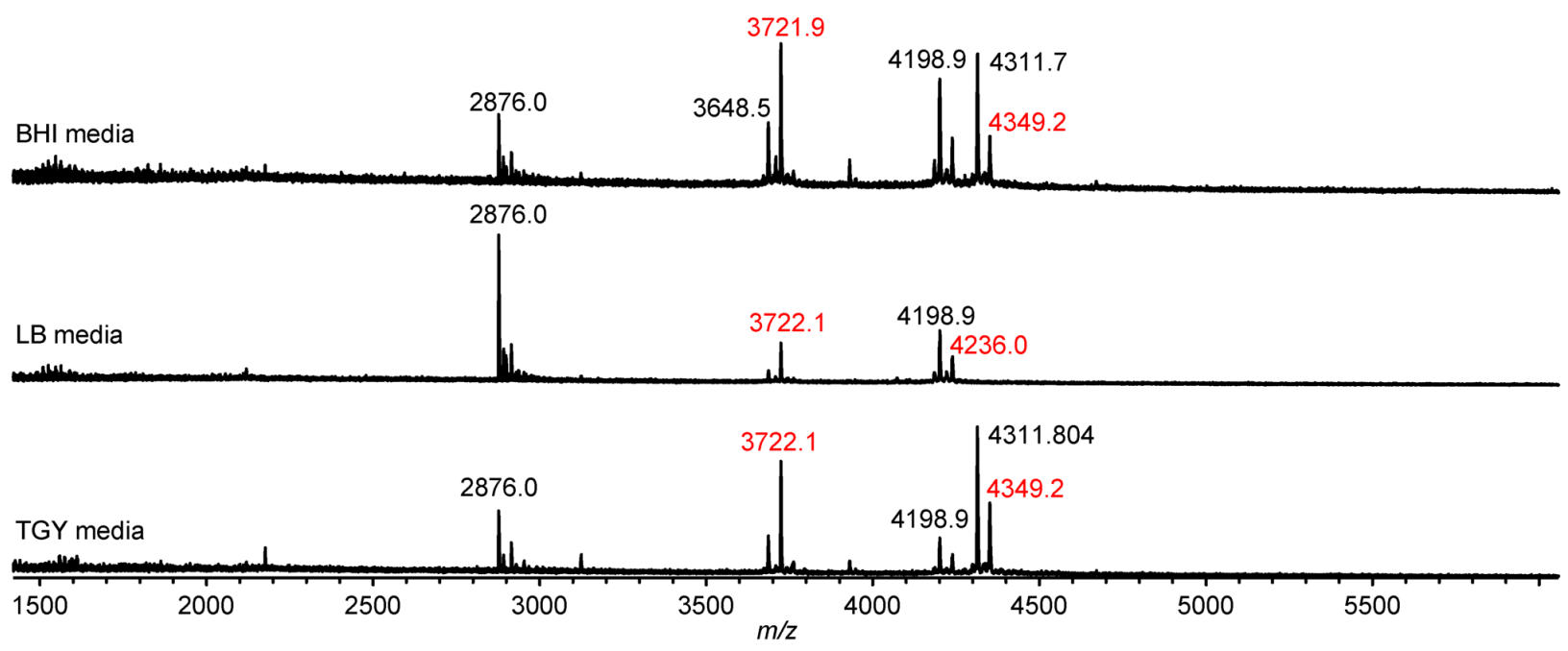

B Huazacin precusor peptide

MEPIQRDDYWGCALKCAGPCLGVCAIDTASPVMDAVGTASGYAGGHG

IQRDDYWGCALKCAGPCLGVCAIDTASPVMDAVGTASGYAGGHG $=4349.0 \mathrm{Da}$ (4 thioethers)

QRDDYWGCALKCAGPCLGVCAIDTASPVMDAVGTASGYAGGHG $=4235.9 \mathrm{Da}$ (4 thioethers)

YWGCALKCAGPCLGVCAIDTASPVMDAVGTASGYAGGHG $=3721.6 \mathrm{Da}$ (4 thioethers) 
Figure S3: HR-ESI-MS/MS of huazacin. (A) Structure of the sactipeptide after undergoing CIDdependent thioether dissociation, characteristic of sactionine linkages, which results in regenerated Cys sulfhydryl groups and dehydro-amino acids at the acceptor residue. ${ }^{24}$ Observed $\mathrm{b}$ and y ions are annotated. (B) $\mathrm{MS} / \mathrm{MS}$ spectrum of the sactipeptide used to localize acceptor amino acids. A previously unseen phenomenon resulting in the loss of $62.0037 \mathrm{Da}$, consistent with formal loss of carbonic acid, is observed for ions containing the Asp acceptor residue. The required decarboxylative dehydration would be similar to known chemistry that occur on carboxylic acid-bearing alkenes. ${ }^{25}$ Error for assigned fragments are listed in Table S6.

A

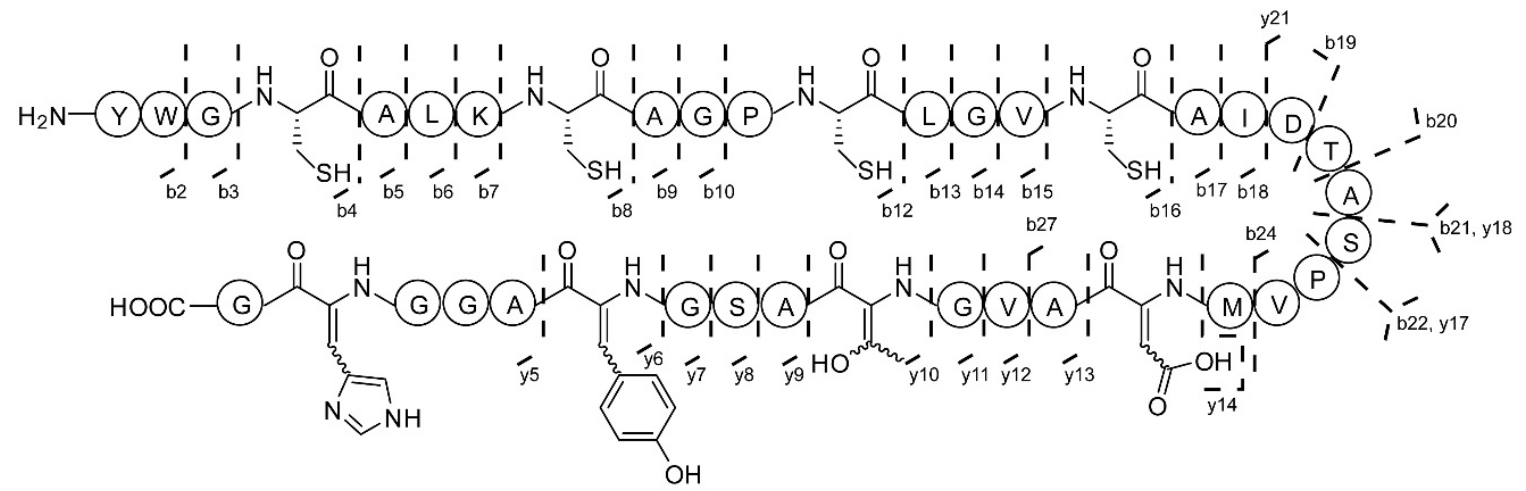

B

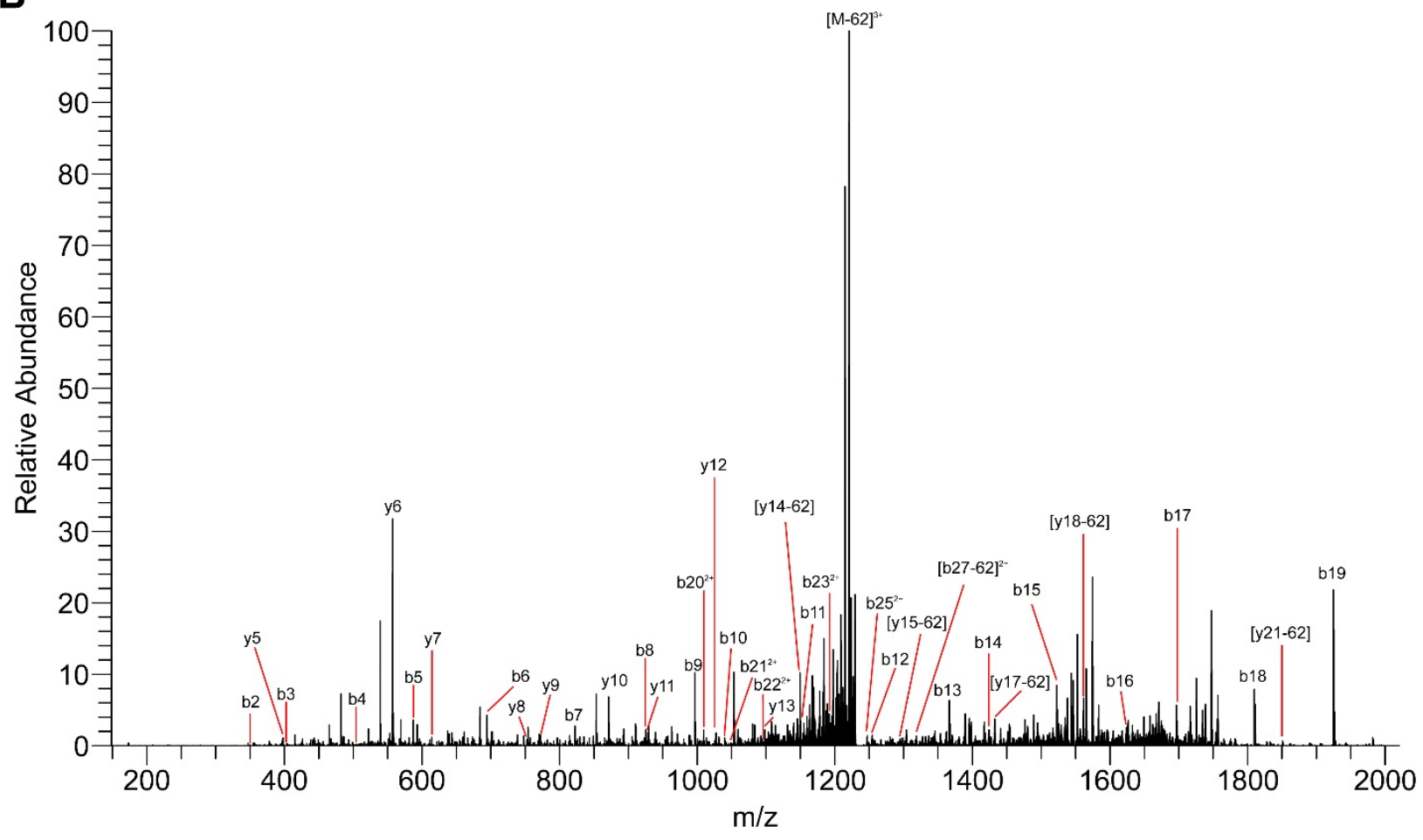


Figure S4: Amide-side chain 1H-TOCSY correlations and assignments of huazacin. Amino acids were identified by canonical TOCSY correlation patterns and assigned sequentially based on NH-NH NOESY correlations. Residues that did not generate NH-NH NOESY correlations were assigned based on side chain correlations. Pro11 and Pro23 did not generate TOCSY correlations due to a lack of protonated amides, and residues Trp2 and Ala35 did not generate amide TOCSY correlations. Thioether receptor residues were assigned based on side chain correlations, with the exception of Asp26, which could not be assigned.

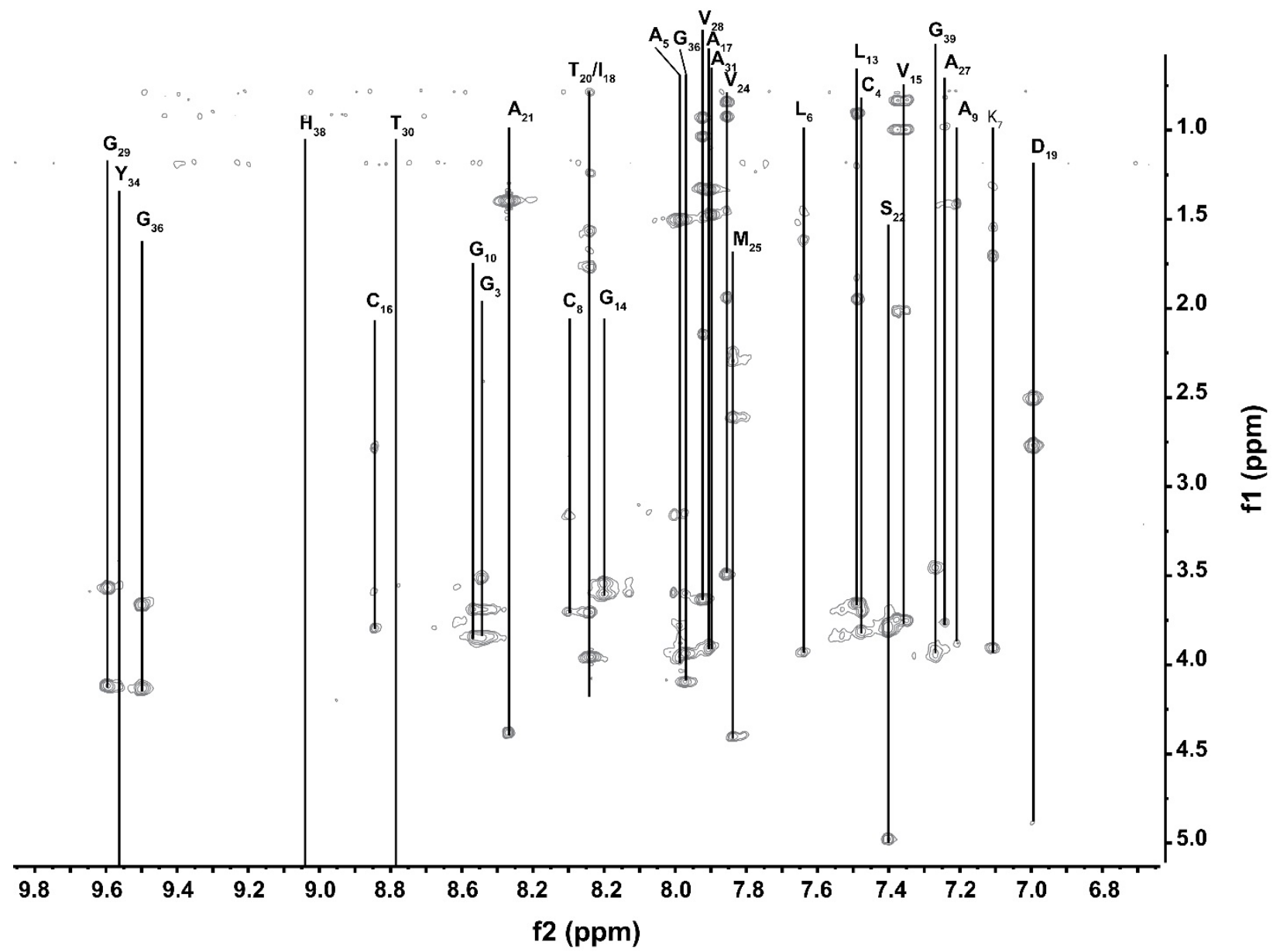


Figure S5: NH-NH NOESY correlations of backbone amides of huazacin. Amino acids are assigned based on their amide proton chemical shifts along the diagonal, and neighboring residue correlations are labelled off-diagonal. Residues that did not generate NOESY correlations were assigned based on the known precursor peptide sequence, process of elimination and side chain proton correlations. While His 38 was confidently assigned by amide-amide correlation, no diagnostic NOESY couplings to Cys4 were found, and Asp26 was not able to be assigned due to a lack of any NOESY correlations to neighboring residues.

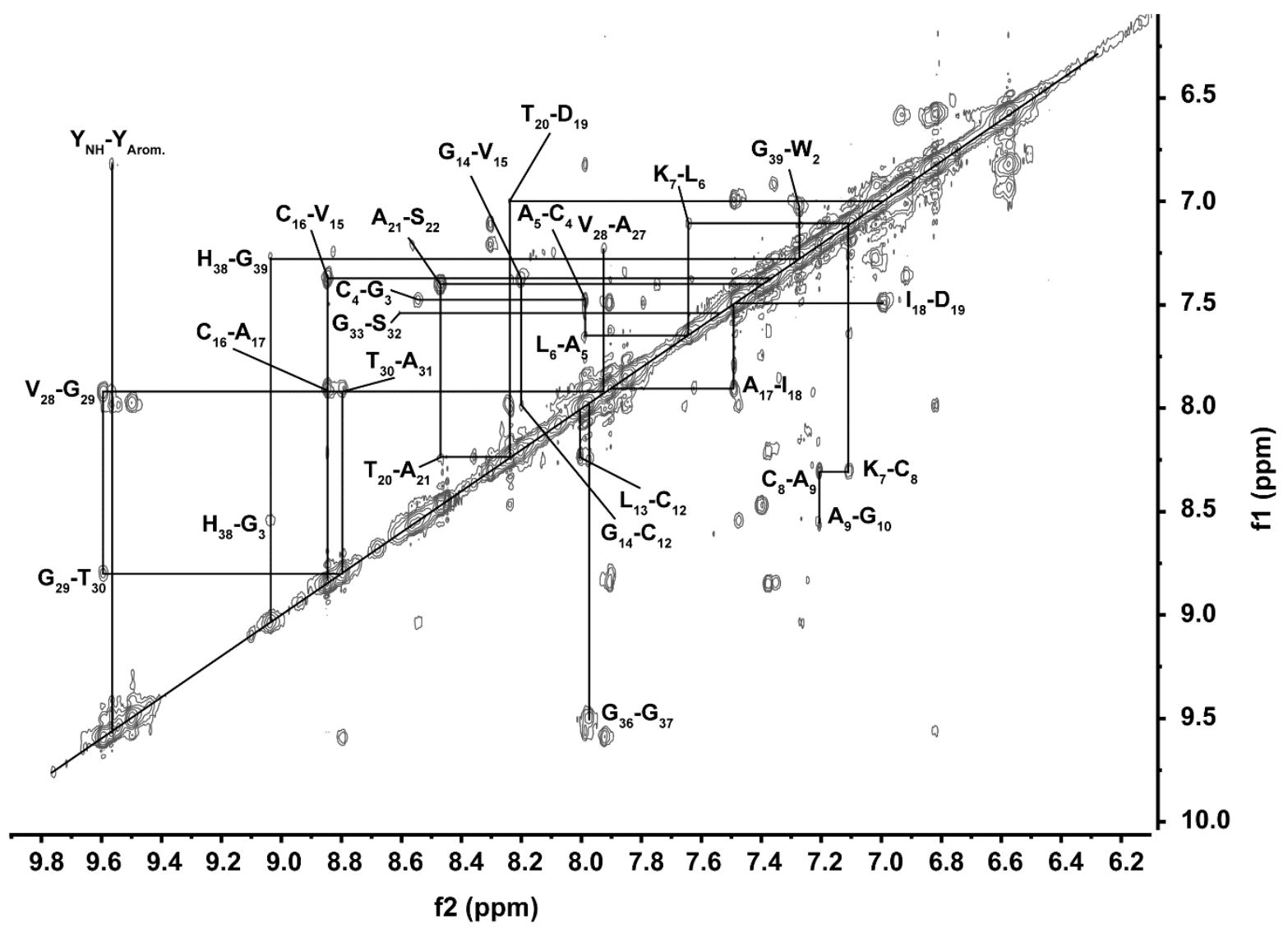


Figure S6: NOESY correlations supporting the S-Ca thioether linkage of huazacin. (A) Structure of the core region of huazacin. (B) Observed NOESY correlations establishing an S-C $\alpha$ linkage. Additional correlations between the Cys and Tyr/Thr side chains are not evident due to long coupling distances and side chain orientations. The absolute stereochemistry of the thioether linkage was not determined.

A

B

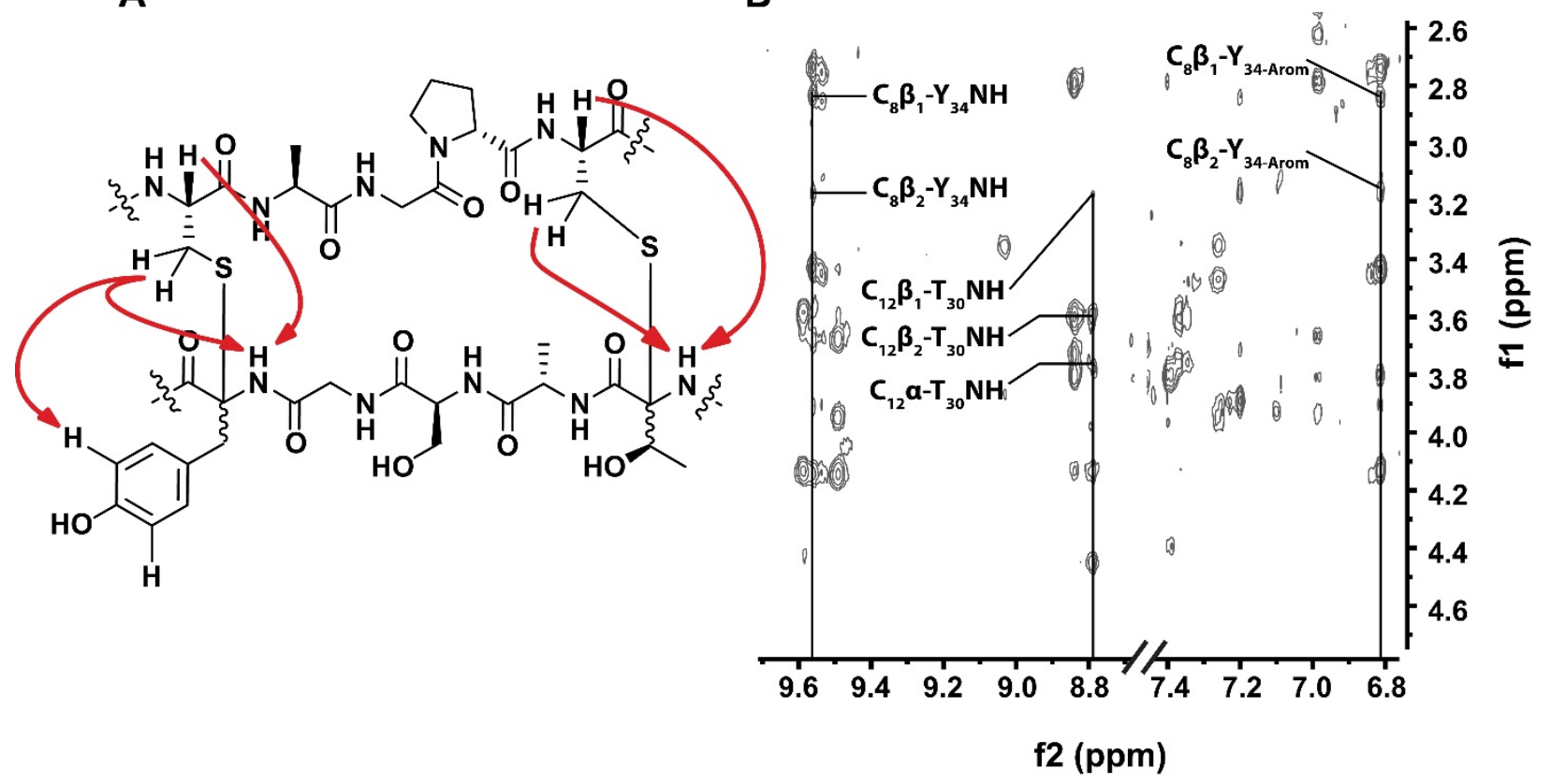


Figure S7: TOCSY correlations of the H-Ca region of huazacin. NMR spectrum used for the assignment of amino acid residues lacking amide-side chain (Pro11, Pro23, Thr30, and Ala35).

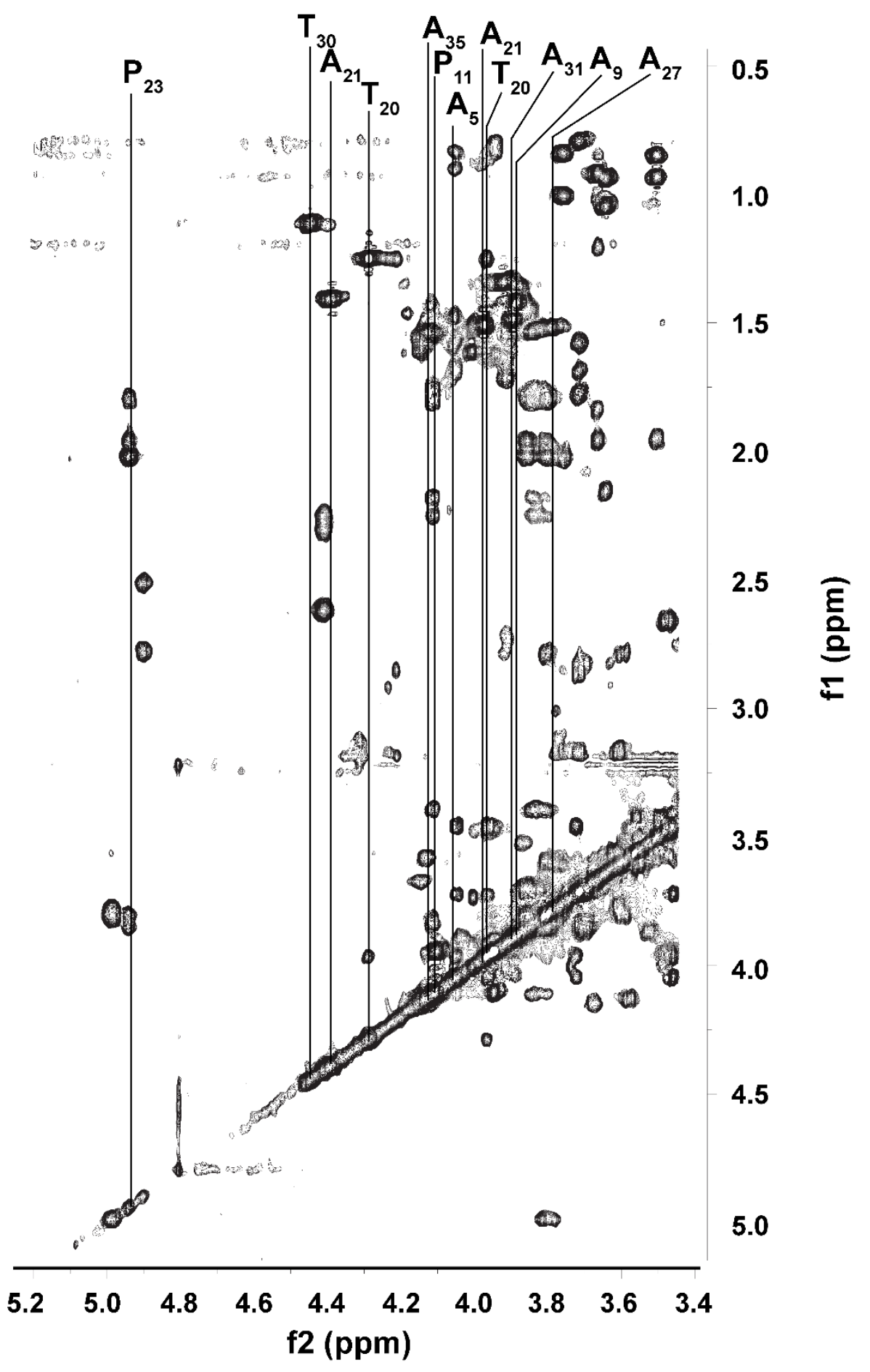


Figure S8: Abundance and sequence characteristics of annotated precursor peptides. (A) The number of rSAM proteins assigned to different groups. (B) The average number of Cys and standard deviation for identified precursor peptides within each major peptide group (see Figures S1 and S9). (C) The Cys length ratio is defined as the distance from the last Cys to the end of the predicted precursor peptide divided by the distance from the first Cys to the final Cys. Ratios $>1$ indicate that a traditional sactipeptide hairpin structure is more likely to form because the distance between the Cys residues is shorter than the length of the remaining core region. Notably SCIFF/ranthipeptides have a ratio $<1$ as their Cys tend to be distributed more evenly. (D) The Cys midpoint ratio represents an average of the positioning of the Cys residues within a precursor peptide. A lower midpoint indicates Cys tend to be more concentrated near the N-terminus of the precursor peptide. HVG, hypervariable group.

A

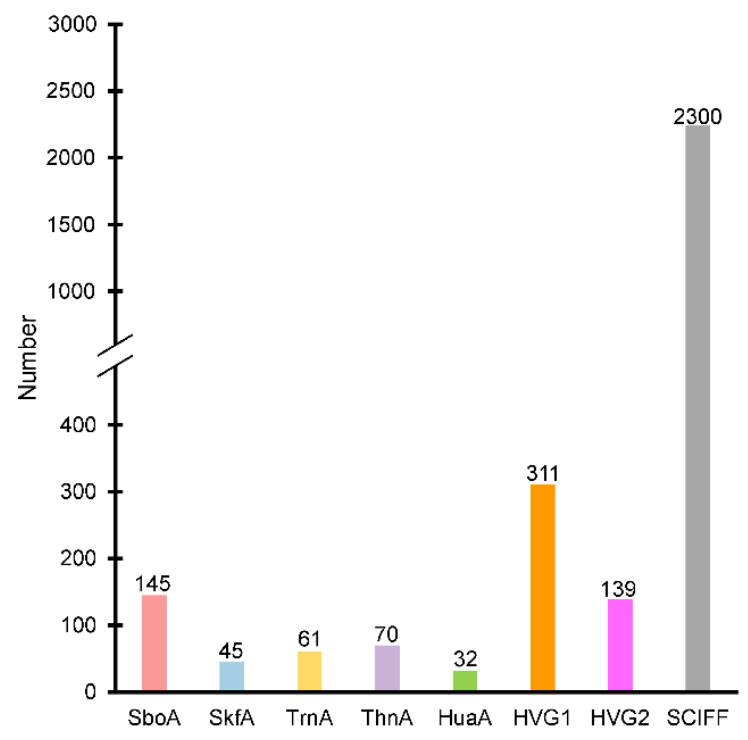

C

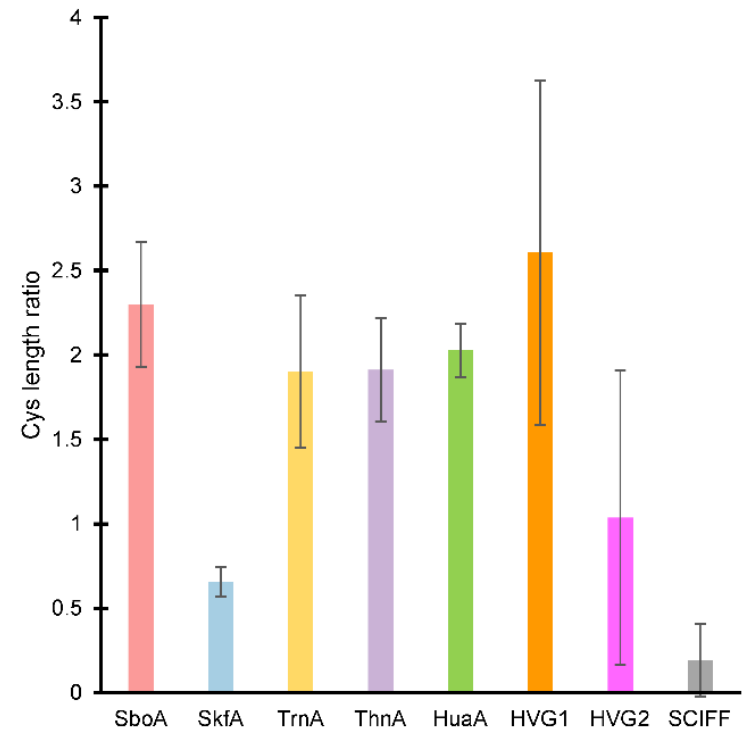

B

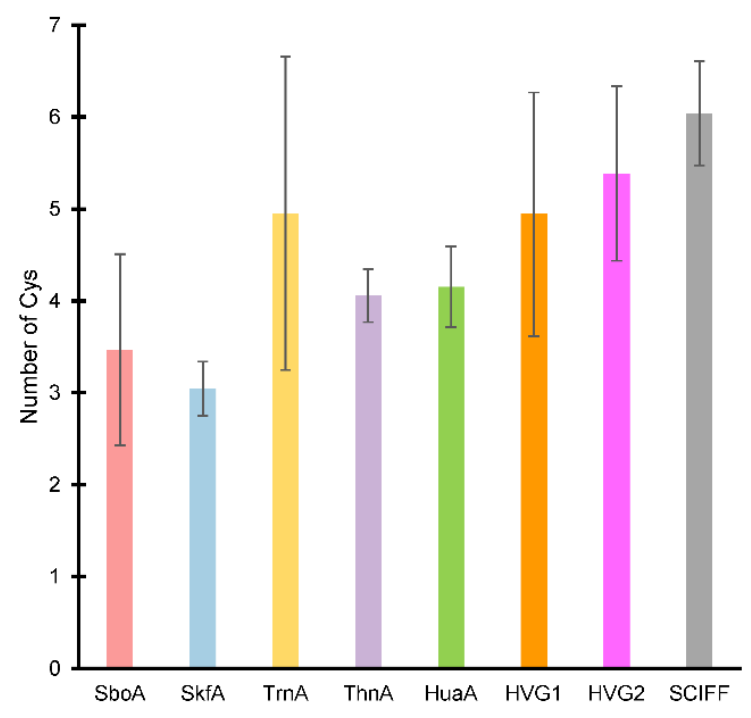

D

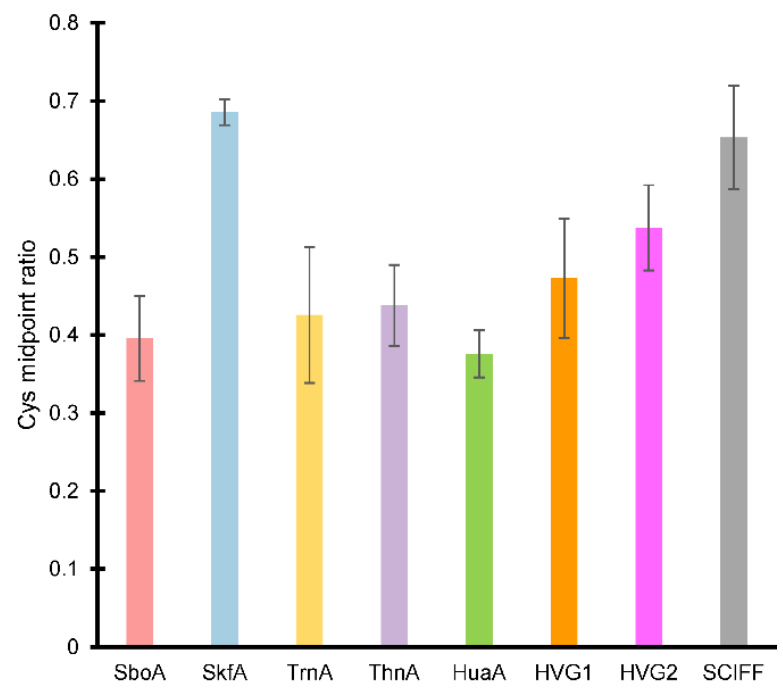


Figure S9: Bioinformatic analysis of SCIFF/ranthipeptide precursor peptides. (A) Precursor peptides form distinct clusters at an alignment score of 8. Nodes are conflated at an identity of $90 \%$, yielding 567 nodes, and colored according to scheme illustrated in panel (B). (B) Histogram of Cys content of identified precursor peptides. As illustrated by the historical name SCIFF (six cysteines in forty-five residues), the vast majority of precursor peptides feature six Cys residues. (C) Sequence logos of the core peptide sequences of the six most populous groups. Groups 2, 3, 5, and 6 have no characterized members.

A
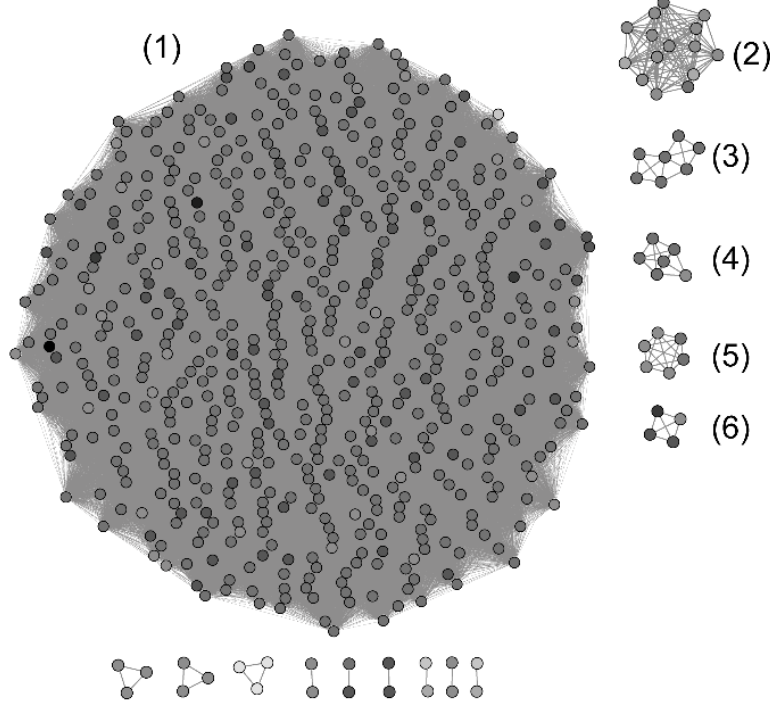

$000000000000000 \bullet 00000000$

000000000000000000

C

(1) thermocellins

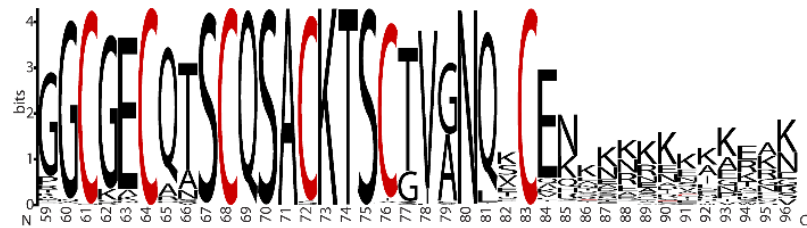

(2) pelrasins [predicted/uncharacterized]

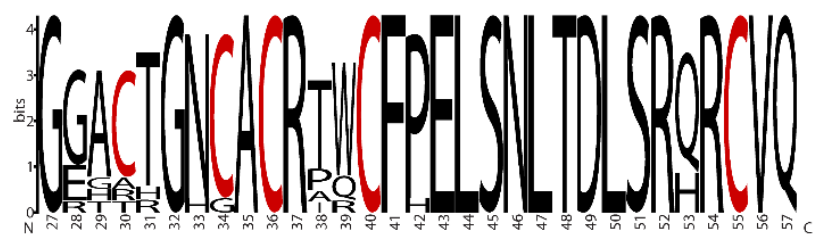

(3) vanirasins [predicted/uncharacterized]

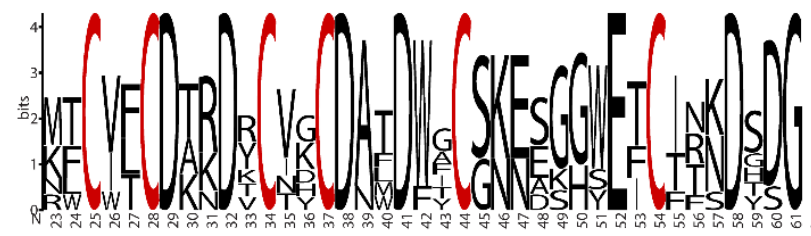

B
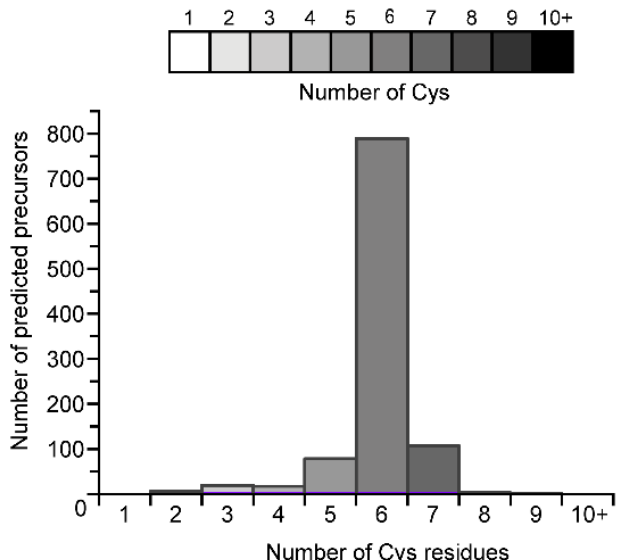

(4) freyrasins

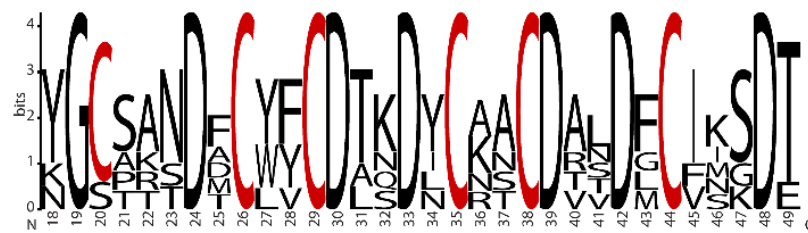

(5) feemasins [predicted/uncharacterized]

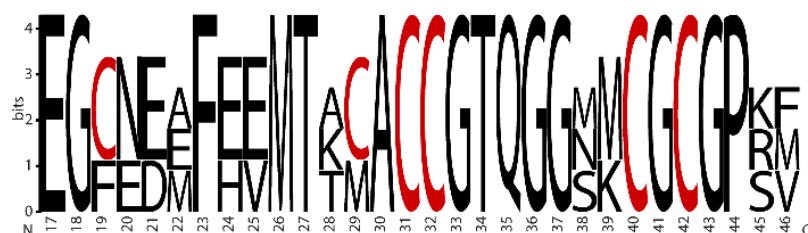

(6) aesirasins [predicted/uncharacterized]

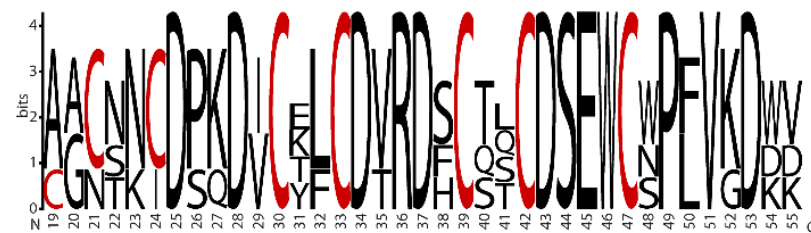


Figure S10: Phylogenetic analysis of organisms containing sactipeptide and SCIFF/ranthipeptide gene clusters. (A) Distribution of identified precursor peptides with accompanying rSAM as identified by RODEO. Phylogenetic data provided by NCBI Taxonomy records indicate that $>95 \%$ originate from Firmicutes. (B) Genus distribution of sequences within Firmicutes with the top 5 most abundant genera denoted.

A

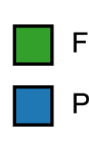

Firmicutes

Proteobacteria

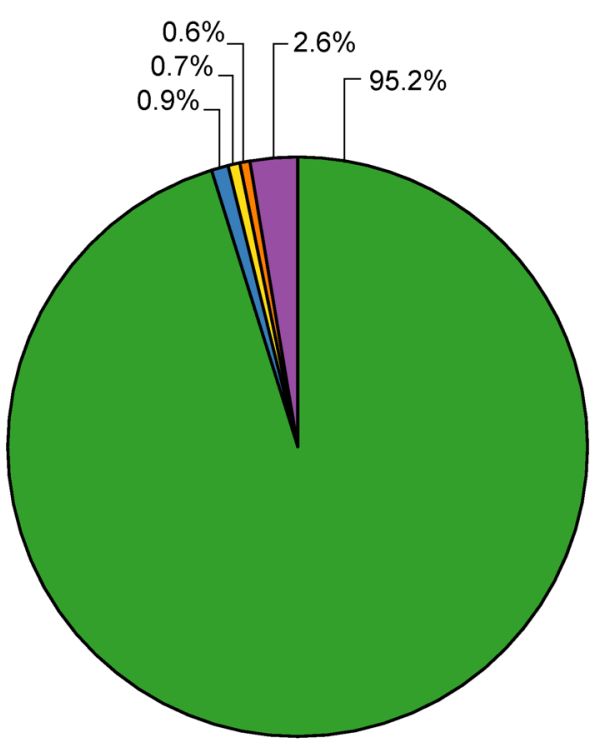

B

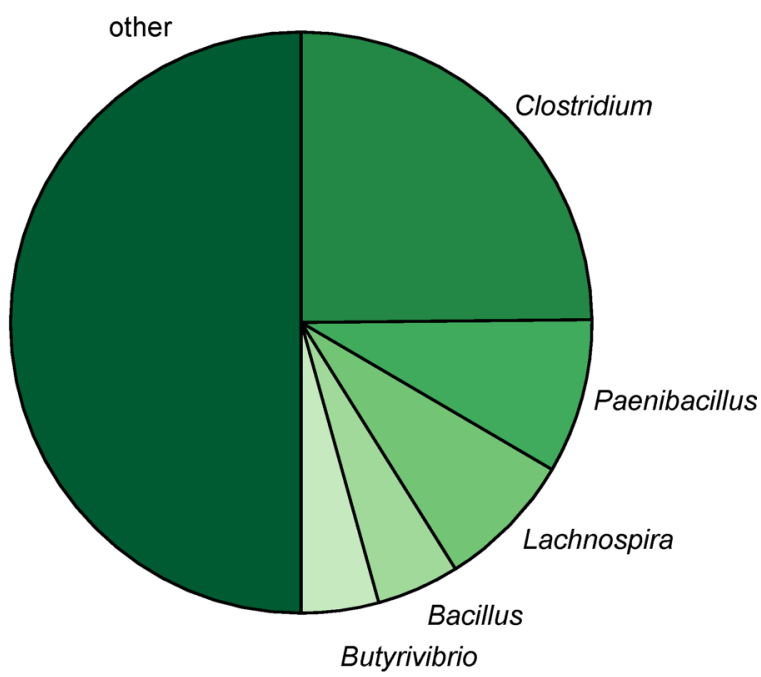


Figure S11: Parametric \% GC analysis of rSAMs. The average \% GC content of the entire genome is compared to the $\% \mathrm{GC}$ of the rSAM gene. The plot contains a selection of known sactipeptides and putative SCIFFs, including those in this report, are highlighted in purple. Regression outliers with significantly higher rSAM \% GC content relative to their genomes (>10\% difference) are highlighted in orange and derive largely from Clostridium.

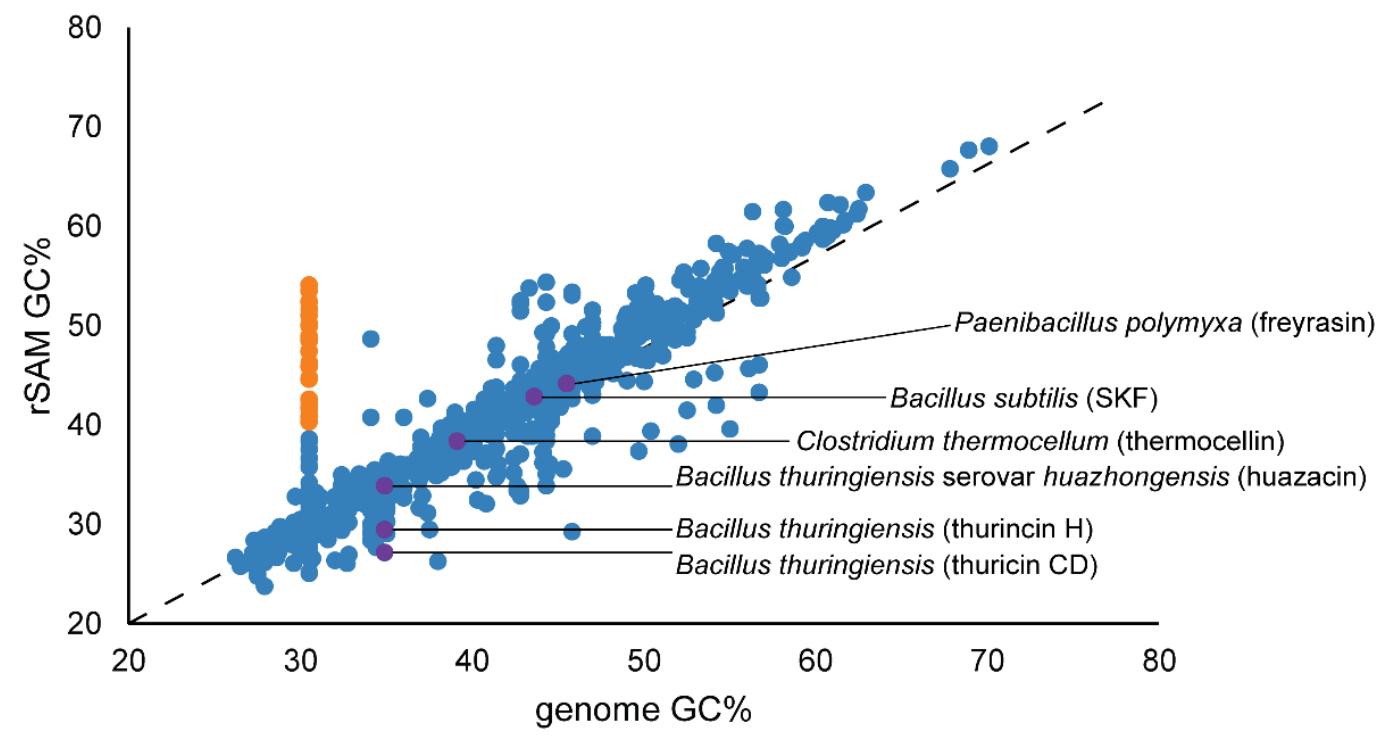


Figure S12: Unusual genomic contexts of SCIFFs. (A) Certain SCIFFs BGCs appear to encode additional genes that may further modify the product (Table S2), such as Orn/Lys/Arg decarboxylase. (B) SCIFFs cooccur with tRNA-related enzymes (Table S2) with high frequency, which might be related to a possible biological role for these natural products. (C) Some biosynthetic operons for quinohemoprotein amine dehydrogenase also feature what appears to be a precursor peptide which may co-opt QhpD to produce a RiPP alongside of quinohemoprotein amine dehydrogenase. Further characterization will be needed to test these hypotheses.

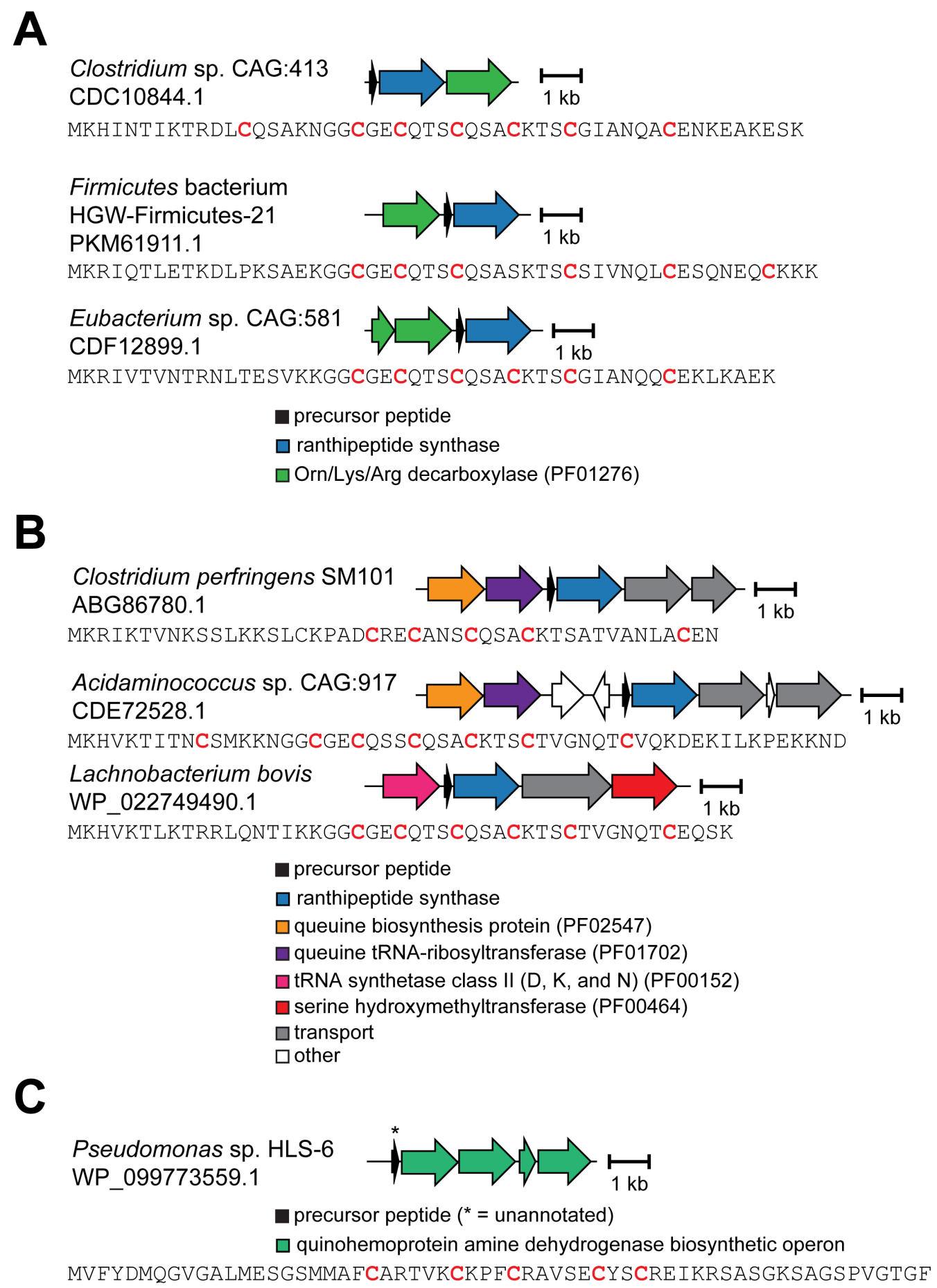


Figure S13: Maximum likelihood tree of rSAMs involved in thioether formation. rSAM enzymes likely involved in thioether installation identified by PSI-BLAST were aligned using the E-INS-i algorithm in MAFFT 7.313 (https://mafft.cbrc.jp/alignment/software/). ${ }^{23}$ While NxxcB, which forms a thioether to the $\beta$-carbon of Asn in a divergent $\mathrm{RiPP}^{26}$ was not part of the BLAST results, it was manually added to gauge similarity to sactipeptide and SCIFF/ranthipeptide enzymes. The MAFFT alignment was used to generate a maximum likelihood tree using FastTree 2.1.10 (http://www.microbesonline.org/fasttree/), which is visualized here using iTOL 4 (https://itol.embl.de/). ${ }^{27,28}$ The tree is rooted using TbtI, a rSAM enzyme involved in thiazole methylation, as an outgroup. ${ }^{29}$ Enzymes involved in $\mathrm{S}-\mathrm{C} \beta$ and $\mathrm{S}-\mathrm{C} \gamma$ thioether formation (blue; PapB, NxxcB, and $\mathrm{CteB}$ ) are closer to $\mathrm{QhpD}$ (green) than any characterized sactionine-forming rSAM (red) as evidenced by sum of appropriate branch lengths. This is further reflected in the percent identity matrix of the labeled rSAMs (Table S7).

Cautionary note: For clarity purposes, this tree omits nearly 40,000 proteins sequences with sequence similarity falling between the sactipeptide and SCIFF/ranthipeptide rSAMs, thus this tree underestimates the true distance between these distinct RiPP classes.

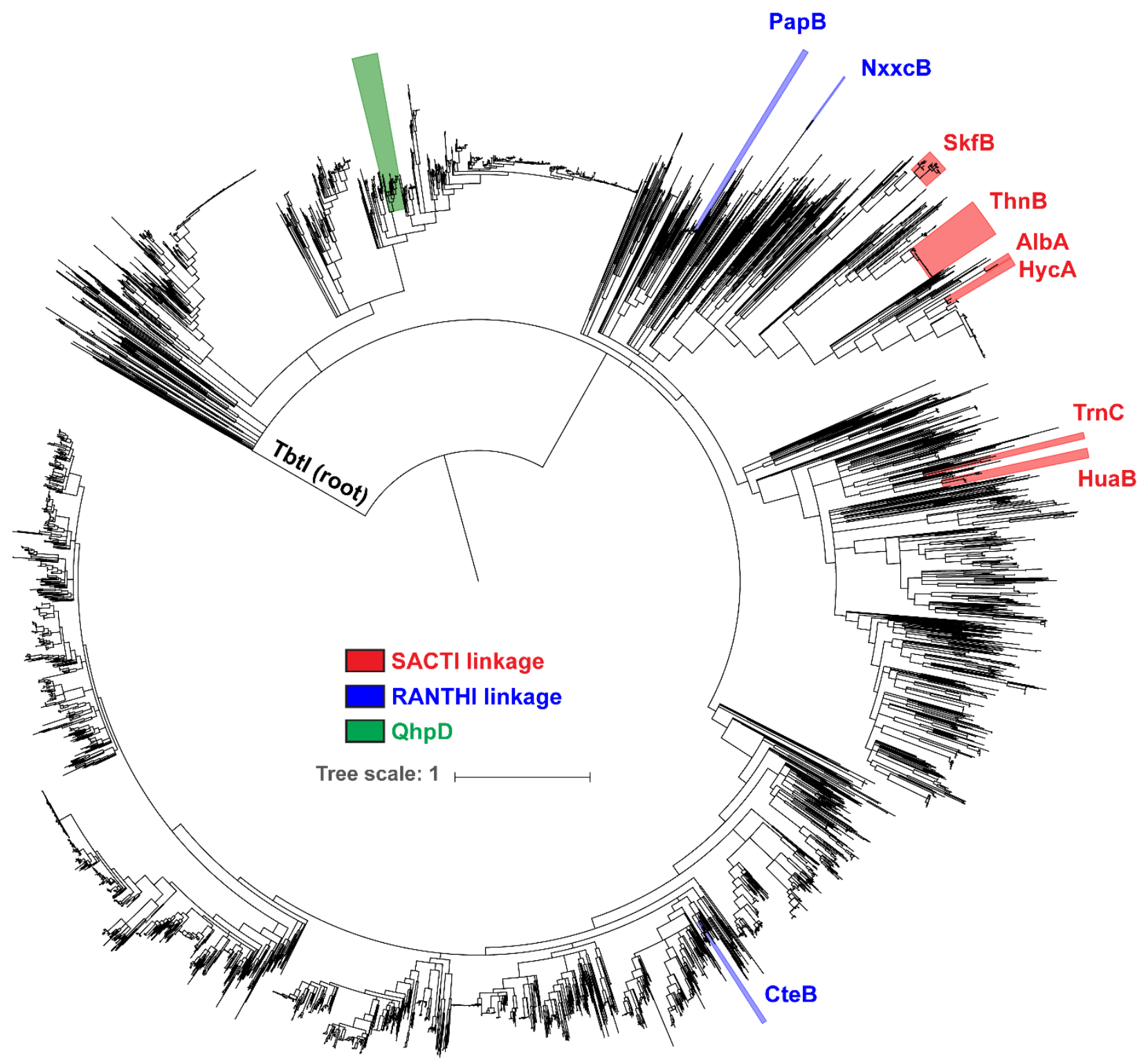


Figure S14: Trypsin digest of the freyrasin precursor peptide. (A) Overview of experimental procedure. (B) When the peptide was expressed without the cognate rSAM enzyme, three disulfide bonds spontaneously formed for a loss of $6 \mathrm{Da}$ (Cys are highlighted yellow). These S-S bonds protected several trypsin sites from digestion. (C) When co-expressed with the cognate rSAM enzyme, the mass of the product was $12 \mathrm{Da}$ lighter than the precursor peptide. Additional mass spectrometry (Figure S15) and NMR spectroscopy (Figures S18-20) supported six Cys-Asp linkages (red and blue, respectively).

A

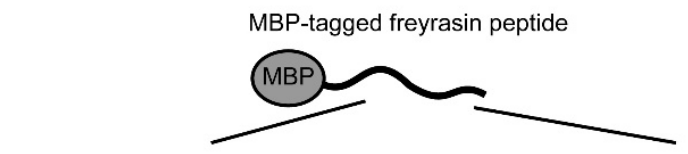

..MLKQINVIAGVKEPIRAYGCSANDACYFCDTRDNCKACDASDFCIKSDT

B

AYGCSANDACYFCDTRDNCKACDASDFCIKSDT

Unmodified $=3576 \mathrm{Da}$

3 disulfide bonds $=3570 \mathrm{Da}$

(1) SCIFF peptide only

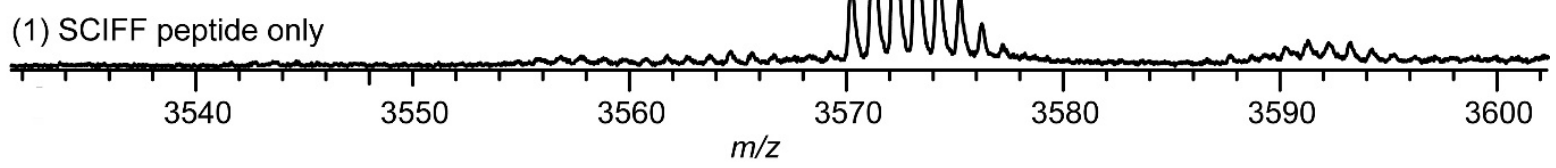

C

AYGCSANDACYFCDTRDNCKACDASDFCIKSDT

Unmodified $=3576 \mathrm{Da}$

6 thioether crosslinks $=3564 \mathrm{Da}$

(2) SCIFF peptide + Radical SAM

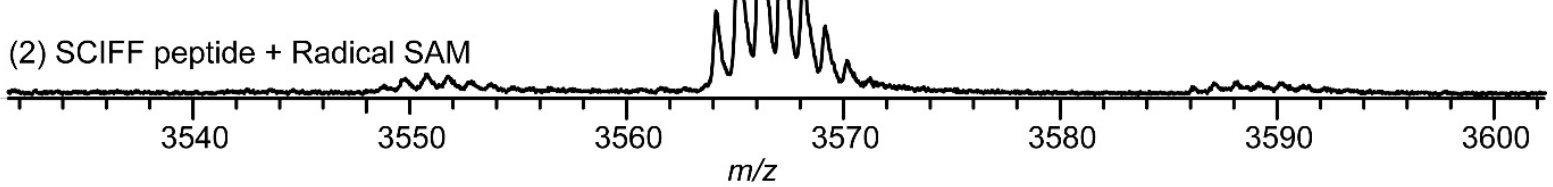


Figure S15: HR-ESI-MS/MS of freyrasin isolated via heterologous expression. (A) Proposed structure of freyrasin after TEV protease treatment to remove the MBP tag with proteolysis occurring at the canonical TEV cleavage site. Observed $b$ and y ions are annotated. (B) CID spectrum of freyrasin $\left([\mathrm{M}+5 \mathrm{H}]^{5+}\right)$. The limited observation of $\mathrm{b}$ and $\mathrm{y}$ ions surrounding the thioether rings of freyrasin is reminiscent of $\mathrm{a}$ lanthionine linkage ( $\beta$-linked thioethers) as fragmentation typically only occurs between rings. ${ }^{30-32}$ Sactipeptides (S-C $\alpha$ thioethers) readily undergo a dissociation reaction which allows for fragmentation within the ring. ${ }^{24}$ Error tables for assigned fragments are in Table S6.

A

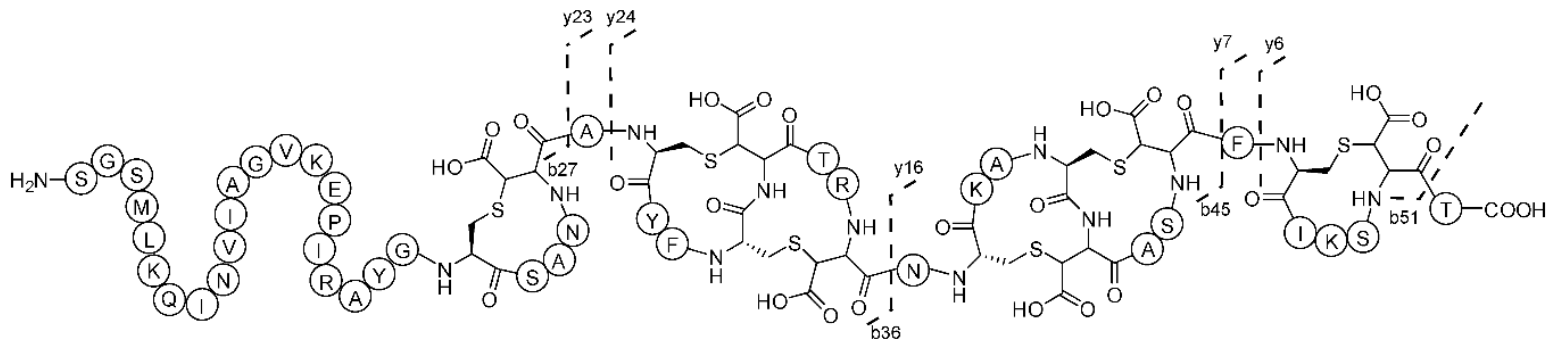

B

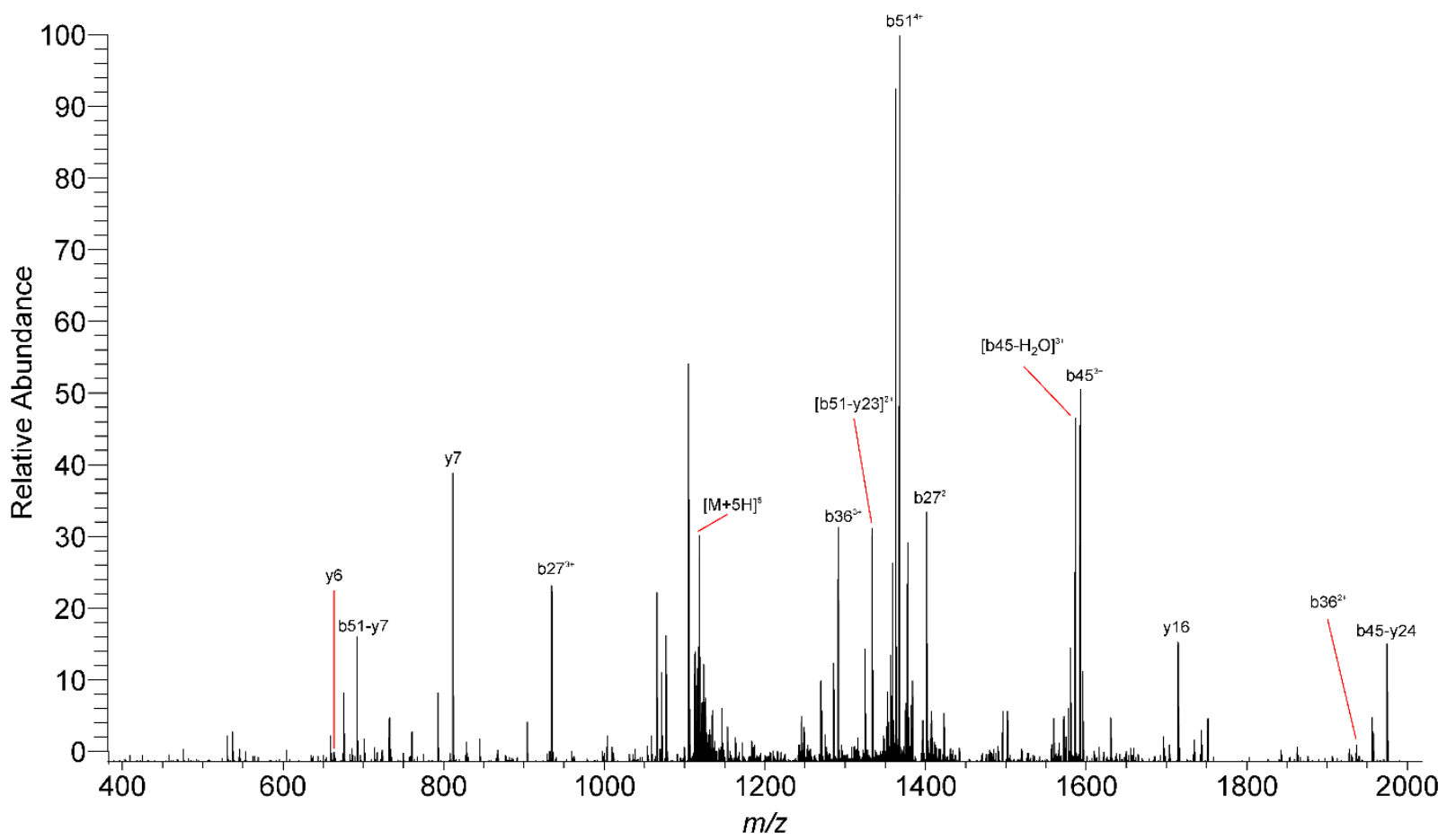


Figure S16: Model of S-Ca and S-C $\boldsymbol{\beta}$ peptides. Two model peptides were used for quantum energy calculations: sulfur bound to the $\alpha$-carbon (A) (S-C $\alpha$ ) or $\beta$-carbon (B) (S-C $\beta$ ). Atom color-coding is as follows: carbon (grey), oxygen (red), nitrogen (blue), sulfur (yellow), and hydrogen (white). (C) Electronic $\left(\mathrm{E}_{\text {elec }}\right)$ and zero-point energies (ZPE) are provided and indicate that $\mathrm{S}-\mathrm{C} \beta$ crosslinks are $12 \mathrm{kcal} / \mathrm{mol}$ more stable than the $\mathrm{S}-\mathrm{C} \alpha$ crosslinked isomer.

A

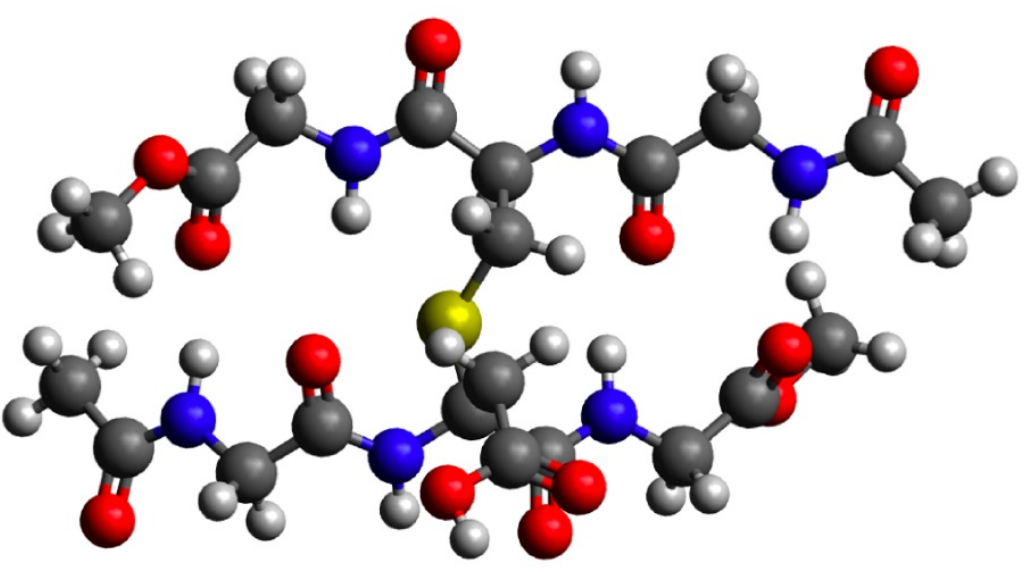

B

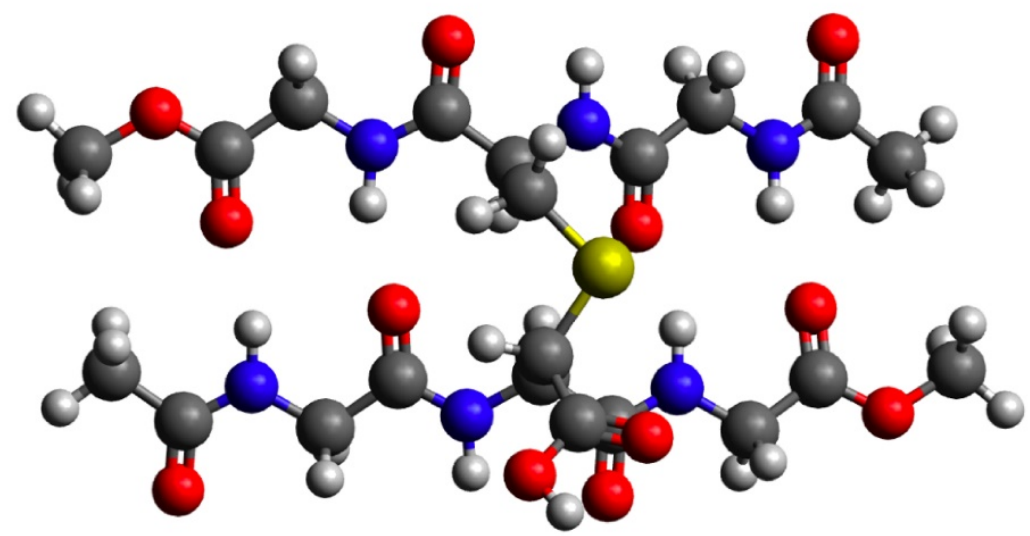

C

\begin{tabular}{|c|c|c|c|}
\hline Energy & $\begin{array}{l}\text { S-Ca } \\
\text { (kcal/mol) }\end{array}$ & $\begin{array}{l}\text { S-CB } \\
\text { (kcal/mol) }\end{array}$ & $\begin{array}{l}\text { Difference } \\
\text { (kcal/mol) }\end{array}$ \\
\hline $\mathrm{E}_{\text {elec }}$ & $1,536,784$ & $1,536,796$ & 12 \\
\hline ZPE & 358 & 357 & 1 \\
\hline
\end{tabular}


Figure S17. Removal of MBP tag and N-terminal residues from expressed freyrasin. (A) Overview of experimental setup. Three peptides arise upon digestion by TEV protease (dashed lines and corresponding masses). TEV protease's canonical sequence and cut site are colored blue. (B) MALDI-TOF-MS spectrum of different samples of freyrasin showing canonical and non-specific digestion of the freyrasin peptide. (C) Fortuitously, the shortest TEV cleaved peptide resulted in most of the predicted leader peptide being removed, which was HPLC-purified and later employed for NMR analysis.

A

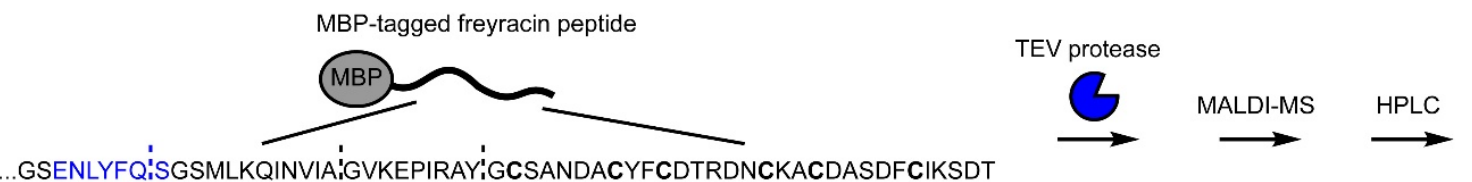

...GSENLYFQ!'SGSMLKQINVIA:GVKEPIRAY'GCSANDACYFCDTRDNCKACDASDFCIKSDT

(1)

(2)

(3)

$5585 \mathrm{Da} \quad 4343 \mathrm{Da} \quad 3330 \mathrm{Da}$

B
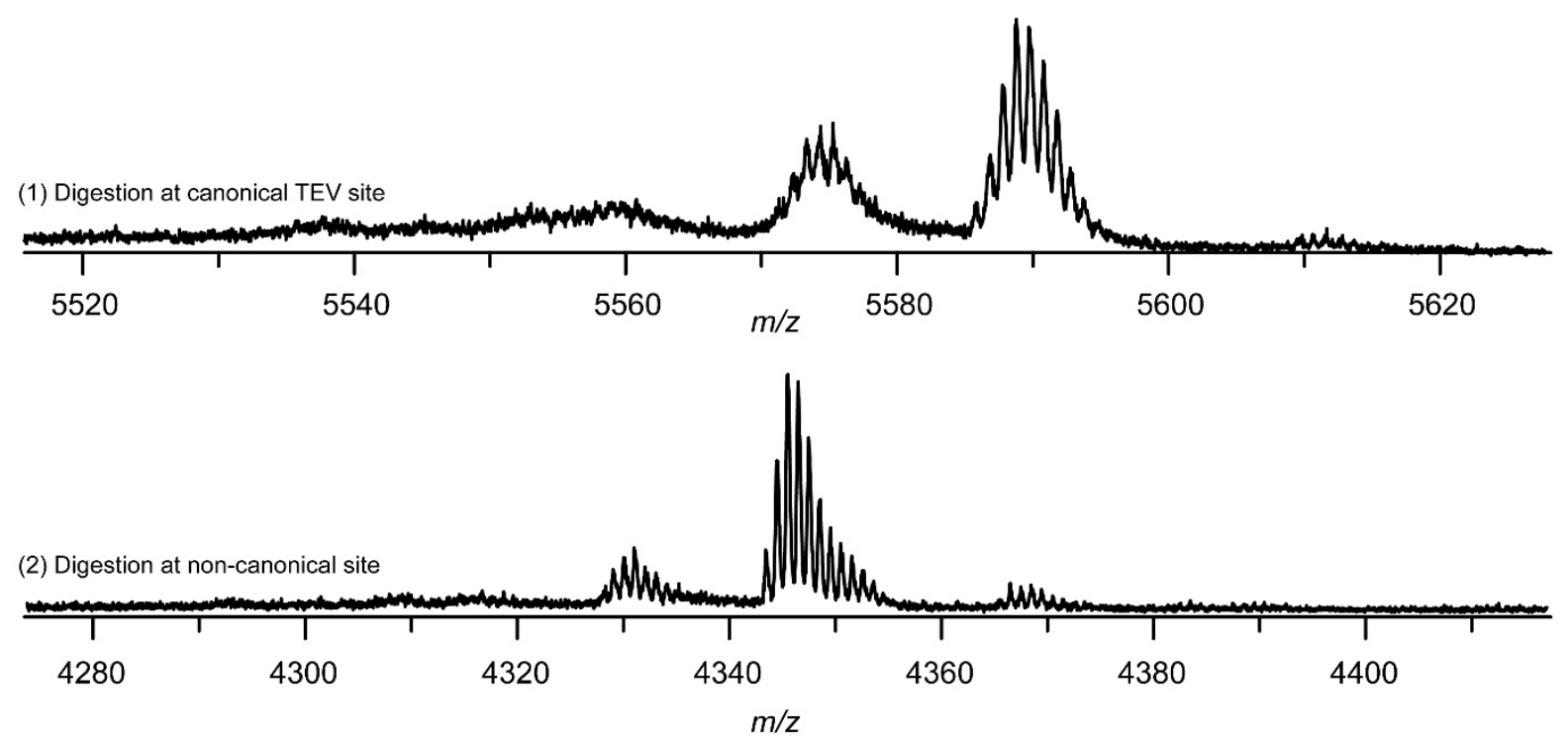

(3) Digestion at non-canonical site

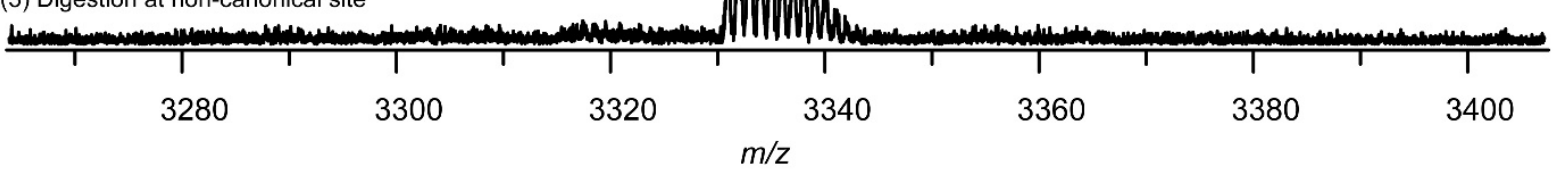

C

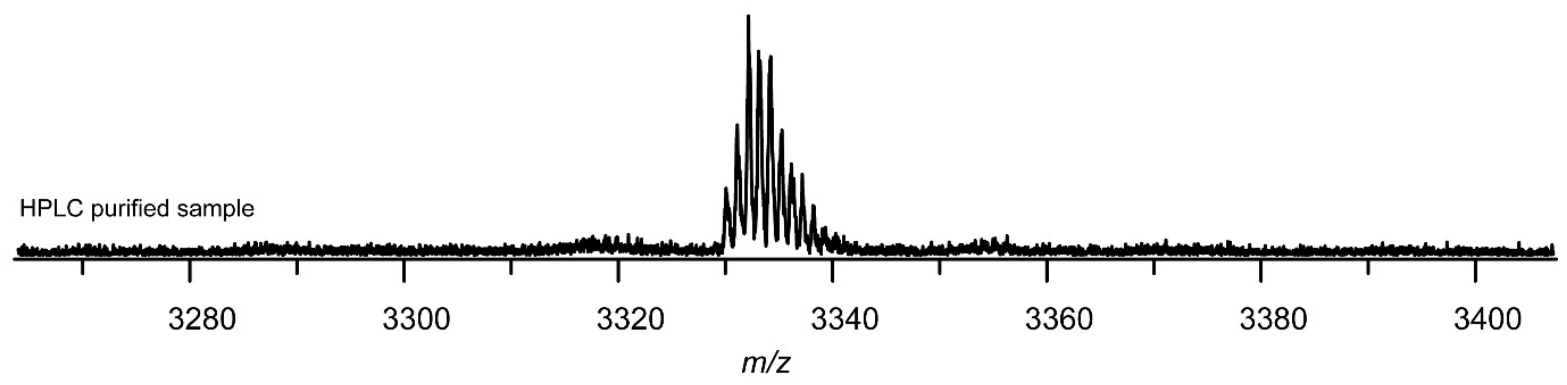


Figure S18: Amide-side chain 1H-TOCSY correlations and assignments of freyrasin. Amino acids were identified by known TOCSY correlation patterns and assigned sequentially based on NH-NH NOESY correlations (see Figure S19). Residues Asn5 and Asn16 were not distinguishable. Residue Cys17 was not observed.

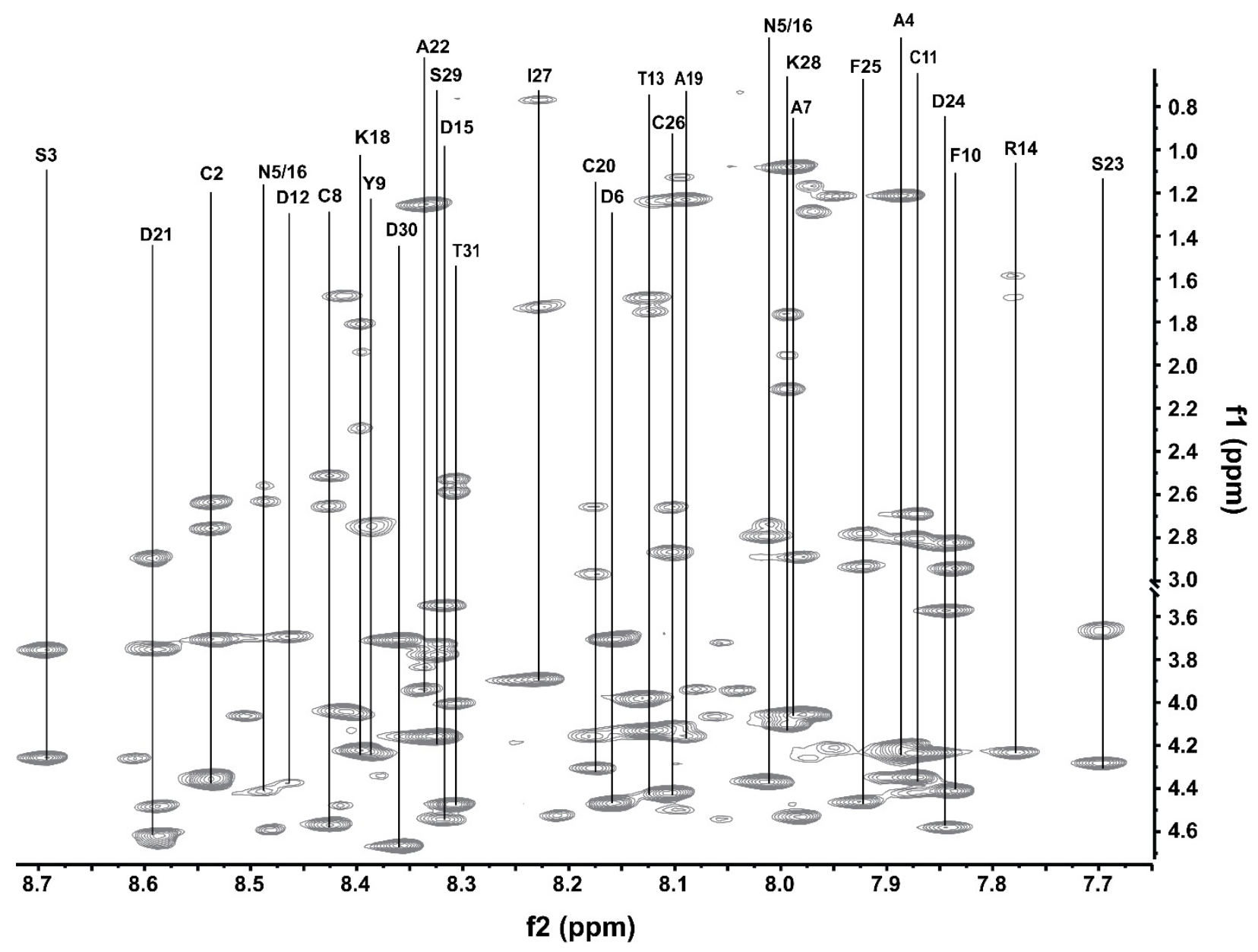


Figure S19: NH-NH NOESY correlations of backbone amides of freyrasin. Amino acids are assigned based on their amide proton chemical shifts along the diagonal, and neighboring residue correlations are labelled off diagonal. Residues that did not generate NOESY correlations were assigned based on the known precursor peptide sequence, process of elimination and side chain proton correlations.

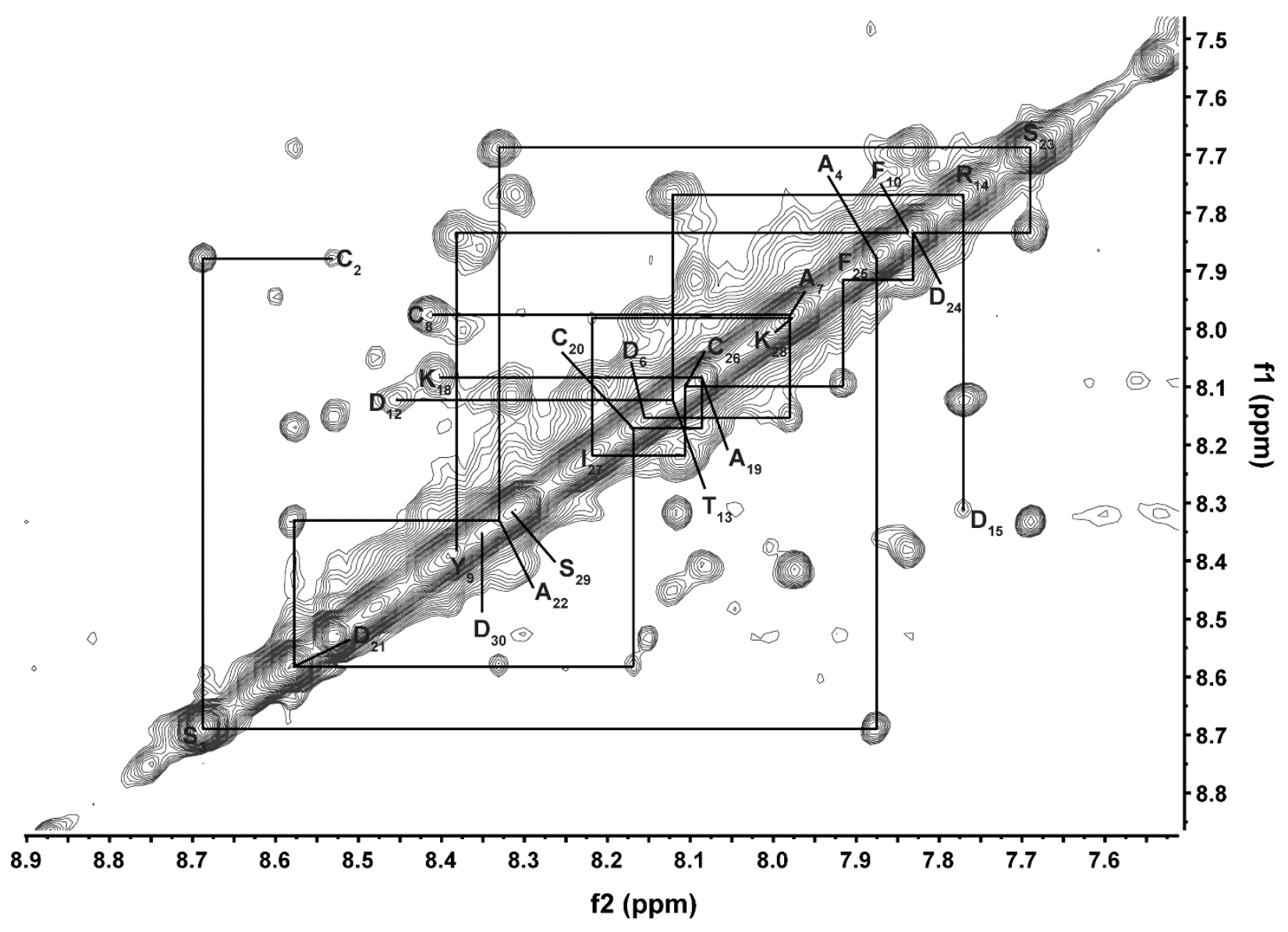


Figure S20: Amide proton-side chain correlations of S-C $\beta$ linked Asp residues of freyrasin. Linked Asp residues demonstrate continuous scalar coupled protons from the amide proton through the side chain protons, a splitting pattern that would not be possible if freyrasin was alpha-linked. Additionally, formation of a thioether bond at Asp C $\beta$ deshields the remaining H $\beta$, generating a TOCSY splitting pattern reminiscent of Ser. Spectral sliced are used to improve clarity.

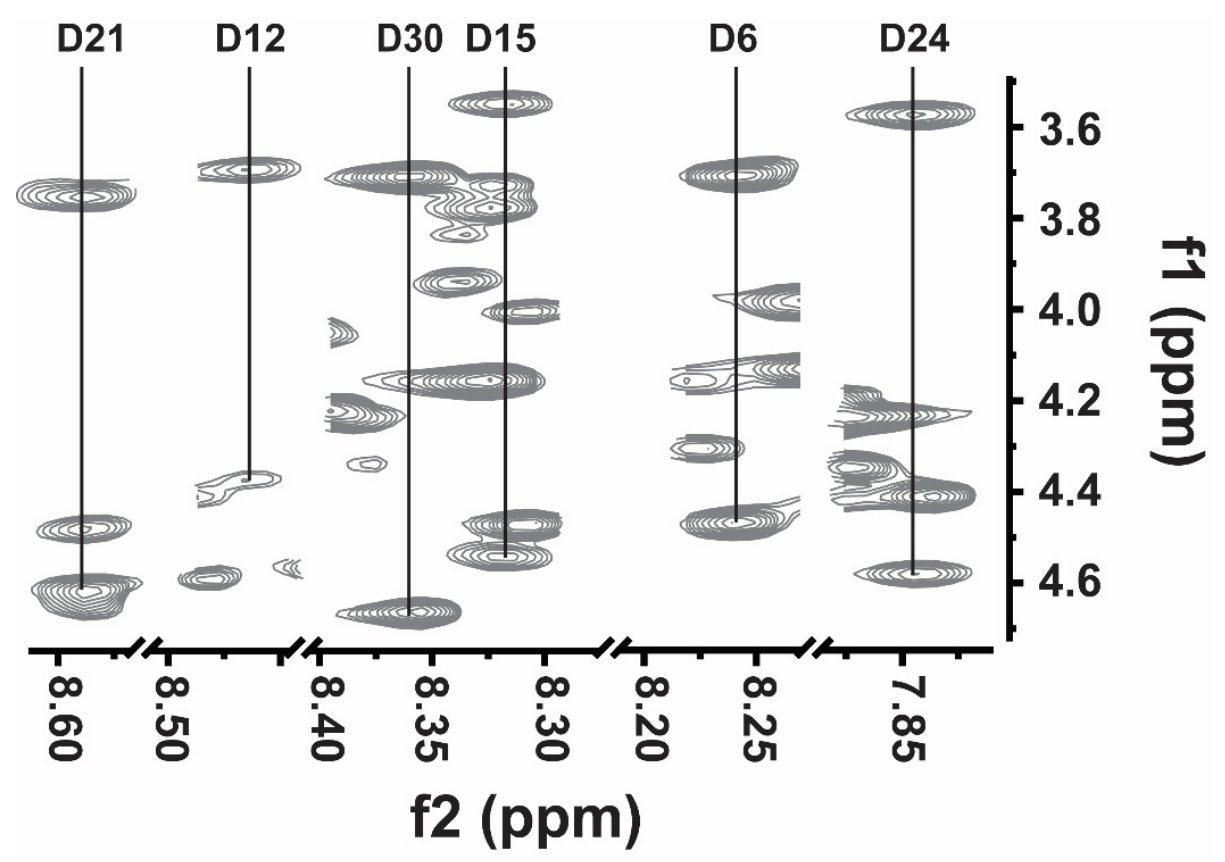


Figure S21: Overexpression of Thr-labeled CteA with Ile and Gly supplementation. (A) Thermocellin precursor peptide, CteA, overexpressed in BL21(DE3) E. coli after purification and removal of the MBP affinity tag. (B) CteA purified from Thr-auxotroph RF2 E. coli grown in defined media supplemented only with $\mathrm{L}-\left[4-{ }^{13} \mathrm{C}, 2,3-{ }^{2} \mathrm{H}_{2}\right]$-Thr results in a large amount of isotopic scrambling from Thr catabolism. ${ }^{20}(\mathbf{C})$ The addition of Ile and Gly suppresses the catabolism of the labeled Thr unit, resulting in near-perfect incorporation of labeled Thr into the CteA precursor peptide. ${ }^{20}$

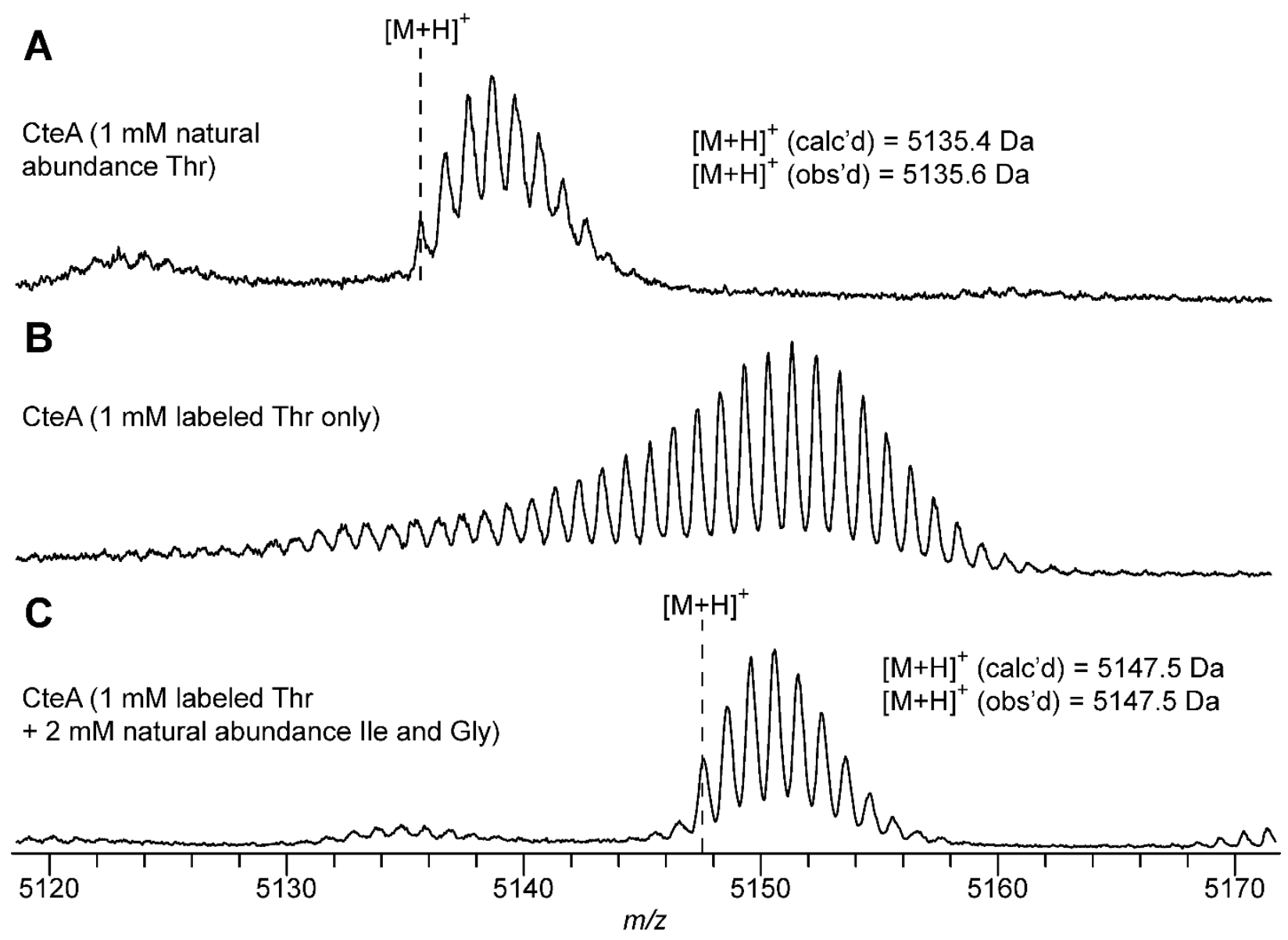


Figure S22: MS/MS localization of isotopic labels to Thr in labeled CteA. (A) GluC treatment was used to remove a majority of N-terminal leader residues and subsequently (B) MS/MS was used to localize isotopic labels exclusively to Thr and showed no detectable scrambling, under-, or over-labeling. Error tables for assigned fragments are in Table S6.

A

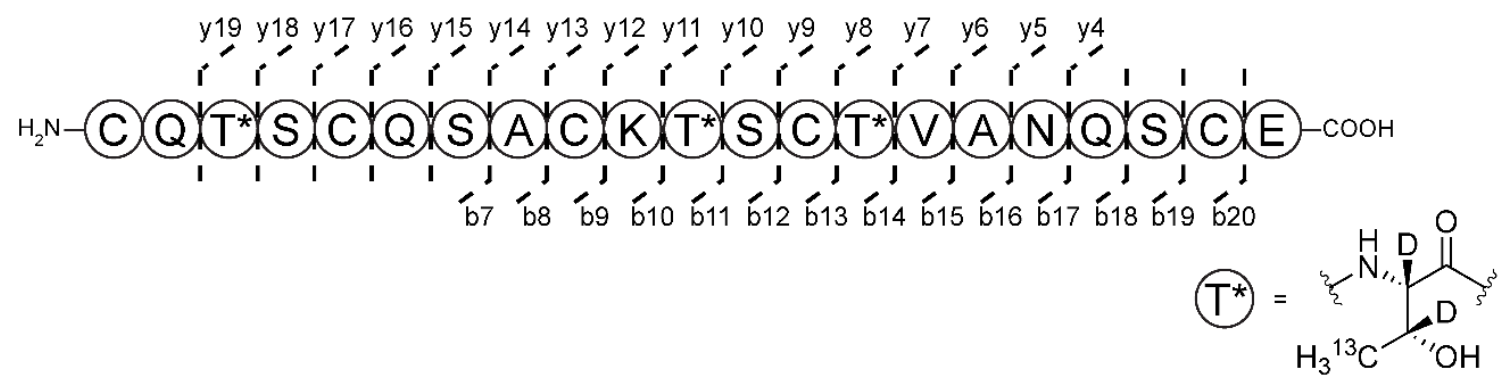

B

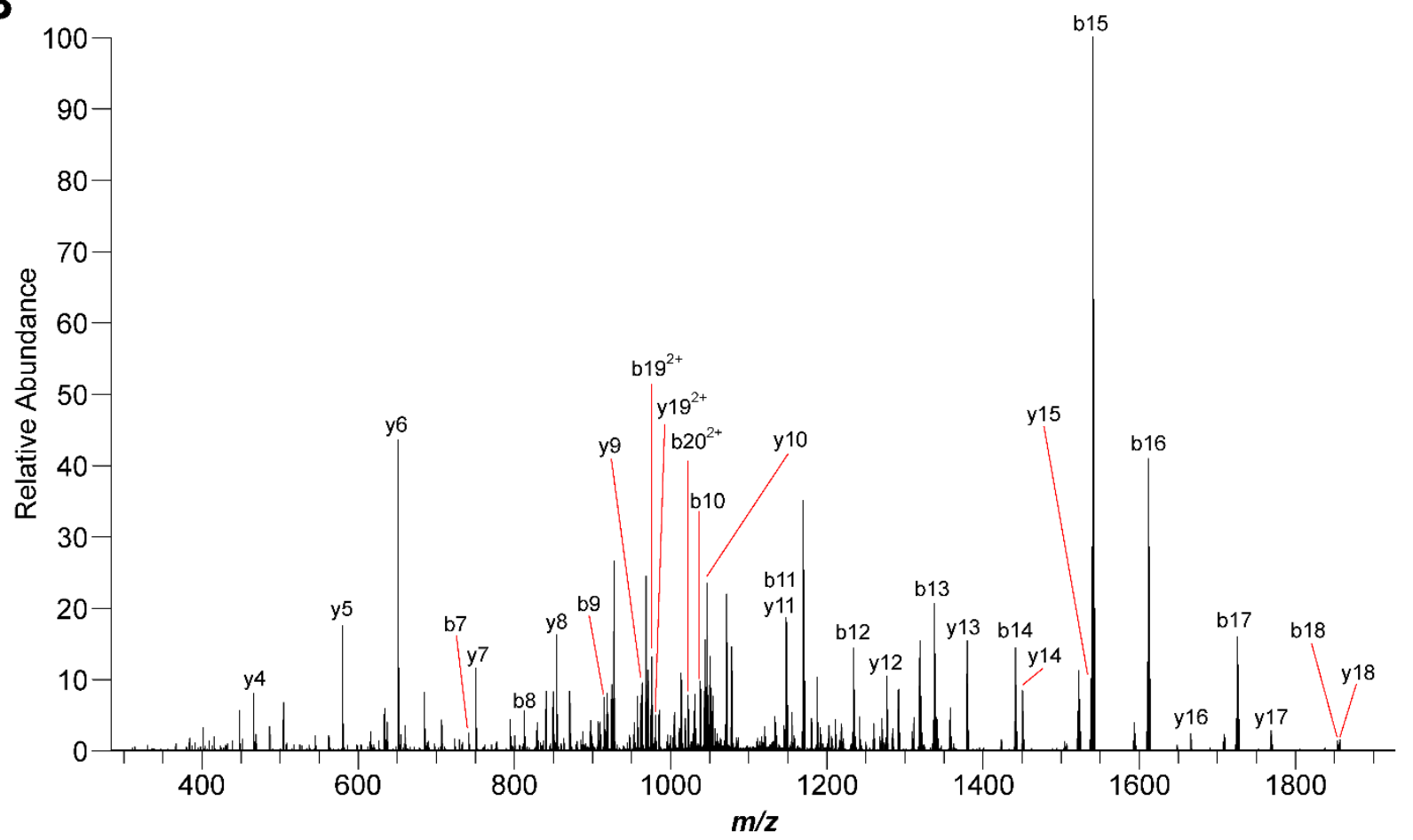


Figure S23: Coexpression of $\mathrm{CteB}$ with labeled $\mathrm{CteA}$ results in ${ }^{\mathbf{1}} \mathrm{H}$ abstraction and $\mathrm{S}-\mathrm{C} \gamma$ thioether formation. Coexpression of $\mathrm{CteB}$ with labeled $\mathrm{CteA}$ under the same conditions that provided ideal labeling exclusively of Thr resulted in a loss of $2 \mathrm{Da}$, consistent with ${ }^{1} \mathrm{H}$ abstraction from the $\gamma$-carbon of Thr and demonstrating the formation of a S-C $\gamma$ thioether.

CteA (1 mM labeled Thr

$+2 \mathrm{mM}$ natural abundance lle and Gly)
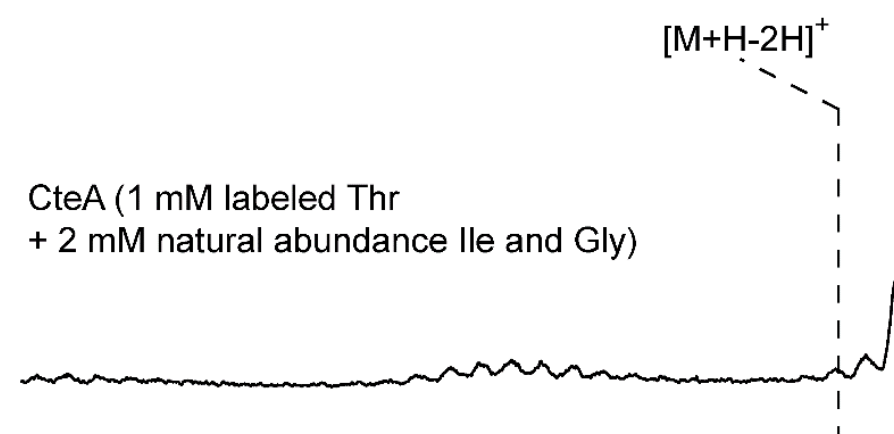

CteA coexpressed with CteB ( $1 \mathrm{mM}$ labeled Thr $+2 \mathrm{mM}$ natural abundance lle and Gly)
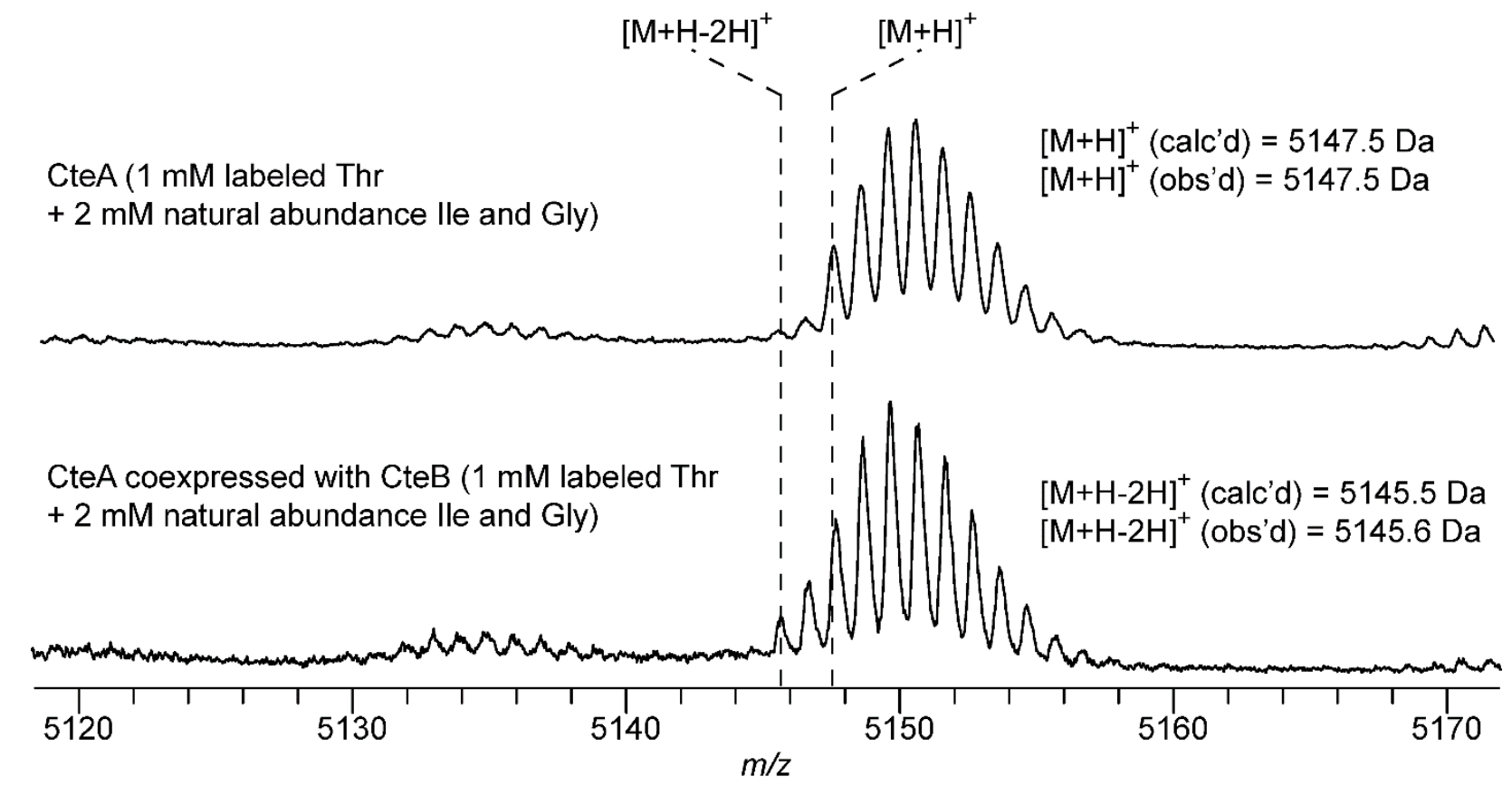
Figure S24: MS/MS analysis of unmodified CteA containing isotopically-labeled Thr. (A) Unmodified peptide was treated with GluC to remove a majority of $\mathrm{N}$-terminal leader residues and subsequently reacted with $\mathrm{N}$-ethylmaleimide to alkylate unmodified Cys. (B) MS/MS analysis after alkylation demonstrates fragmentation within the macrocycle observed in modified thermocellin (Figure 6), specifically ions y13, y14, and b10. Error tables for assigned fragments are in Table S6.

A

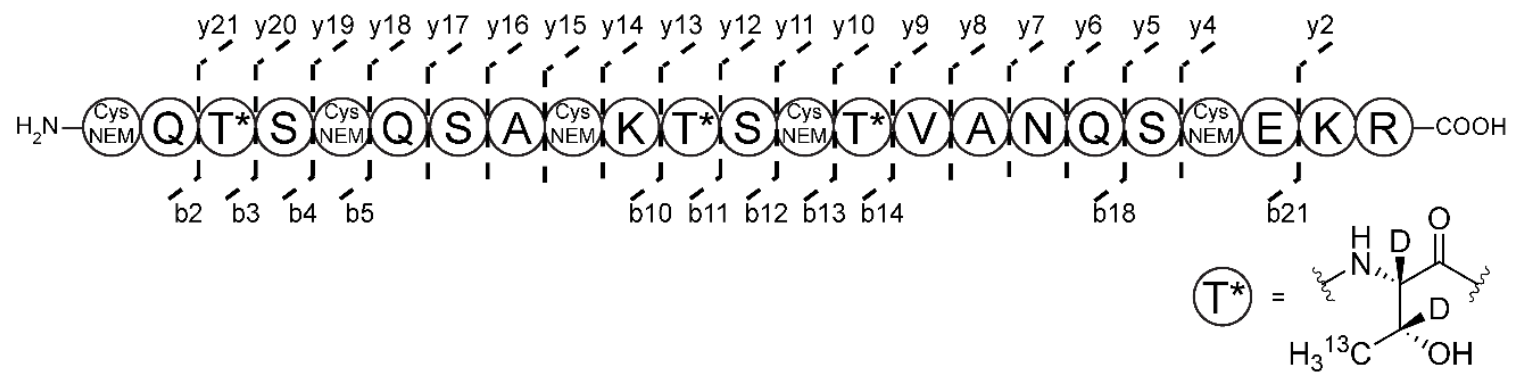

B

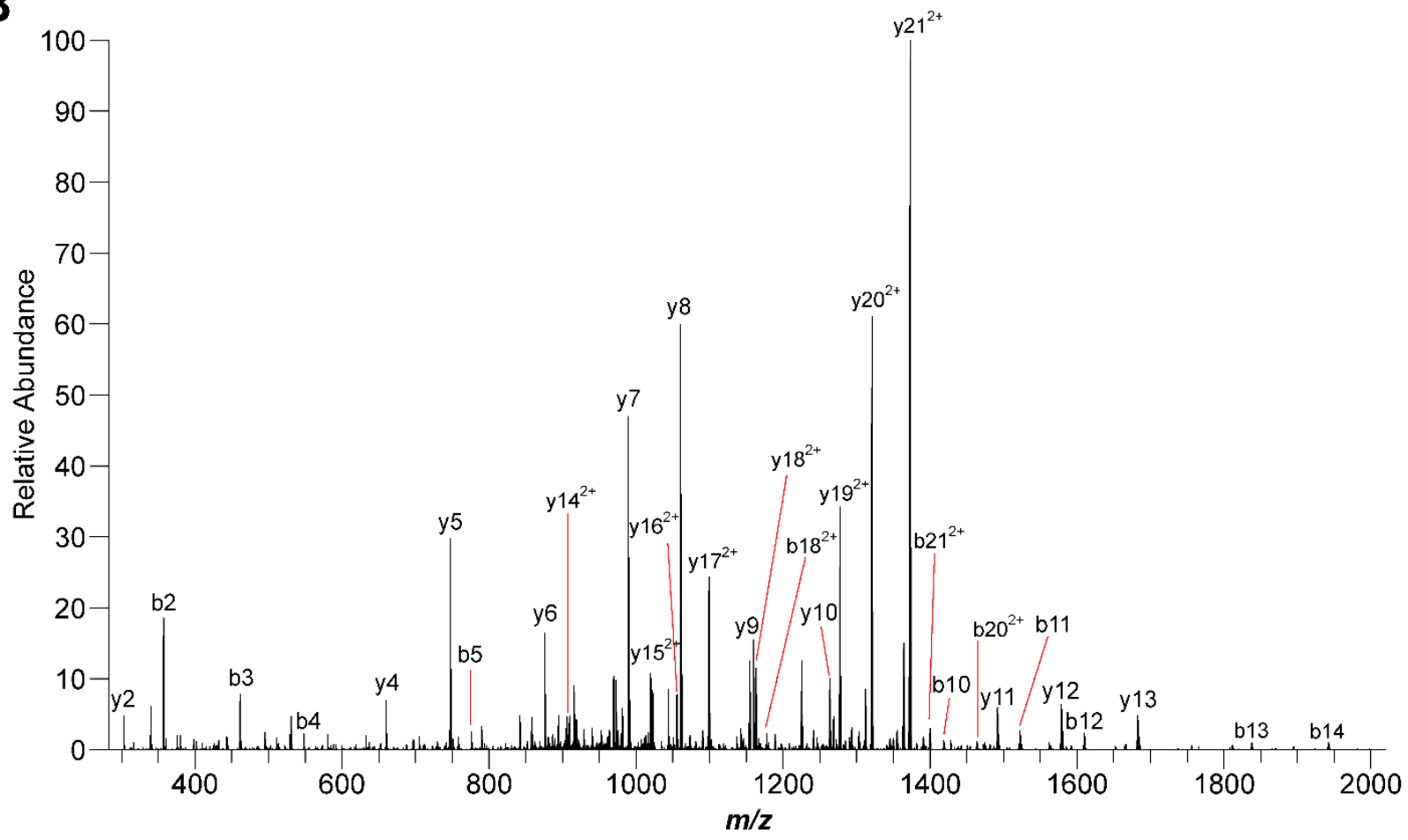


Figure S25: MS/MS analysis of modified CteA containing natural abundance Thr. (A) Modified coexpression product was treated with GluC to remove a majority of $\mathrm{N}$-terminal leader residues and subsequently reacted with $N$-ethylmaleimide to alkylate unmodified Cys. (B) MS/MS analysis demonstrates that the thioether macrocycle still localizes to the same position as when the precursor peptide is isotopically labeled at Thr (Figure 6). Error for assigned fragments are provided in Table S6.

A

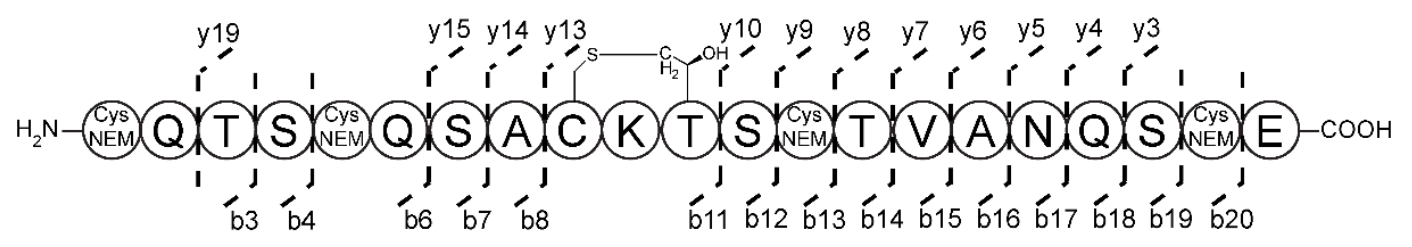

B

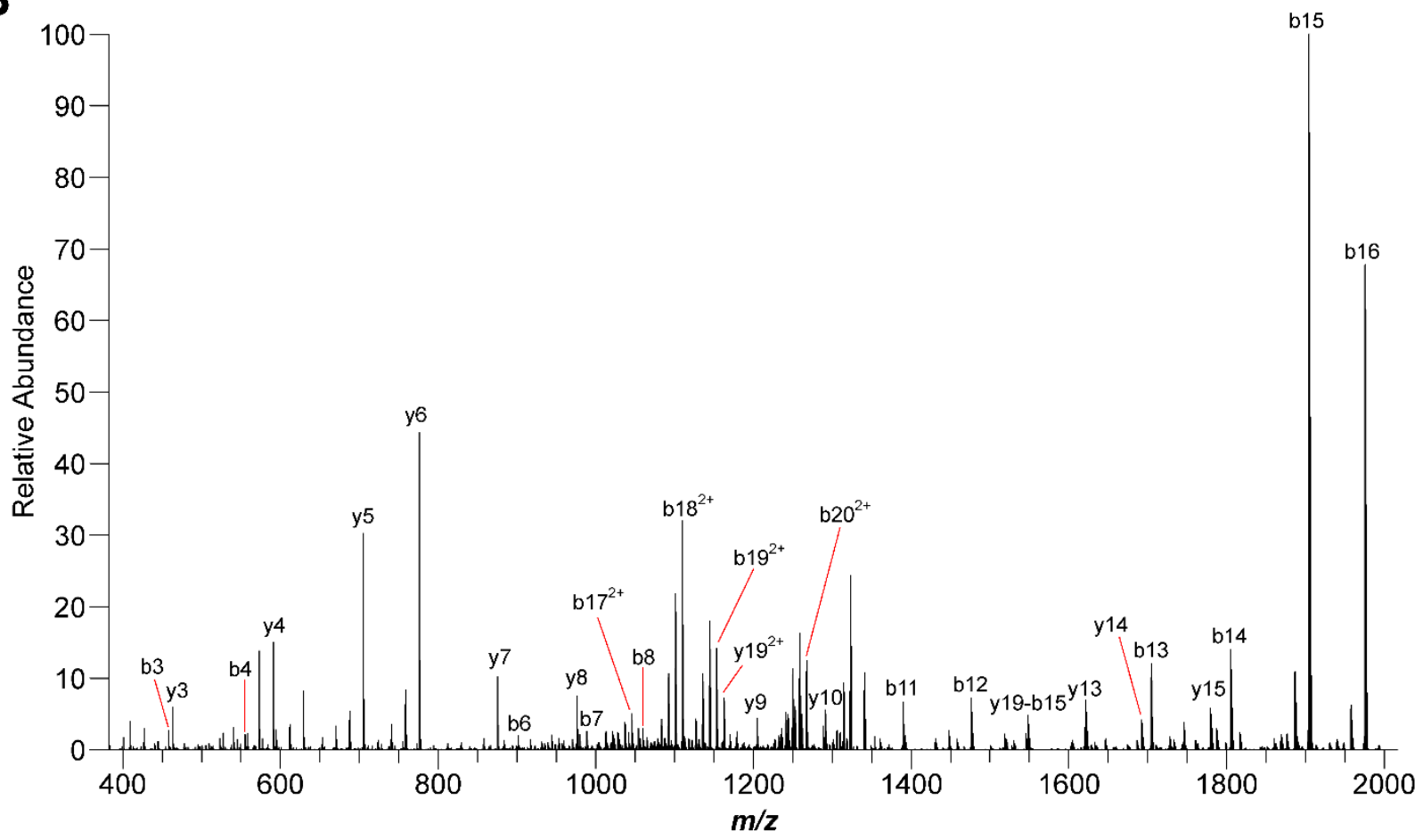




\section{Supplemental References}

(1) Altschul, S. F.; Madden, T. L.; Schaffer, A. A.; Zhang, J.; Zhang, Z.; Miller, W.; Lipman, D. J. Gapped BLAST and PSI-BLAST: a new generation of protein database search programs. Nucleic Acids Res. 1997, 25 (17), 3389.

(2) Flühe, L.; Marahiel, M. A. Radical S-adenosylmethionine enzyme catalyzed thioether bond formation in sactipeptide biosynthesis. Curr. Opin. Chem. Biol. 2013, 17 (4), 605.

(3) Grove, T. L.; Himes, P. M.; Hwang, S.; Yumerefendi, H.; Bonanno, J. B.; Kuhlman, B.; Almo, S. C.; Bowers, A. A. Structural insights into thioether bond formation in the biosynthesis of sactipeptides. J. Am. Chem. Soc. 2017, 139 (34), 11734.

(4) Bruender, N. A.; Wilcoxen, J.; Britt, R. D.; Bandarian, V. Biochemical and spectroscopic characterization of a radical S-adenosyl-1-methionine enzyme involved in the formation of a peptide thioether cross-link. Biochemistry 2016, 55 (14), 2122.

(5) Tietz, J. I.; Schwalen, C. J.; Patel, P. S.; Maxson, T.; Blair, P. M.; Tai, H.-C.; Zakai, U. I.; Mitchell, D. A. A new genome-mining tool redefines the lasso peptide biosynthetic landscape. Nat. Chem. Biol. 2017, $13(5), 470$.

(6) Kans, J. In Entrez Programming Utilities Help; National Center for Biotechnology Information: Bethesda, MD, 2013.

(7) Cock, P. J.; Antao, T.; Chang, J. T.; Chapman, B. A.; Cox, C. J.; Dalke, A.; Friedberg, I.; Hamelryck, T.; Kauff, F.; Wilczynski, B.; de Hoon, M. J. Biopython: freely available Python tools for computational molecular biology and bioinformatics. Bioinformatics 2009, 25 (11), 1422.

(8) Blin, K.; Wolf, T.; Chevrette, M. G.; Lu, X.; Schwalen, C. J.; Kautsar, S. A.; Suarez Duran, H. G.; de Los Santos, E. L. C.; Kim, H. U.; Nave, M.; Dickschat, J. S.; Mitchell, D. A.; Shelest, E.; Breitling, R.; Takano, E.; Lee, S. Y.; Weber, T.; Medema, M. H. antiSMASH 4.0-improvements in chemistry prediction and gene cluster boundary identification. Nucleic Acids Res. 2017, 45 (W1), W36.

(9) Su, G.; Morris, J. H.; Demchak, B.; Bader, G. D. Biological network exploration with Cytoscape 3. Curr. Protoc. Bioinformatics 2014, 47, 8131.

(10) Kohl, M.; Wiese, S.; Warscheid, B. Cytoscape: software for visualization and analysis of biological networks. Methods Mol. Biol. 2011, 696, 291.

(11) Eddy, S. R. Profile hidden Markov models. Bioinformatics 1998, 14 (9), 755.

(12) Finn, R. D.; Coggill, P.; Eberhardt, R. Y.; Eddy, S. R.; Mistry, J.; Mitchell, A. L.; Potter, S. C.; Punta, M.; Qureshi, M.; Sangrador-Vegas, A.; Salazar, G. A.; Tate, J.; Bateman, A. The Pfam protein families database: towards a more sustainable future. Nucleic Acids Res. 2016, 44 (D1), D279.

(13) Himes, P. M.; Allen, S. E.; Hwang, S.; Bowers, A. A. Production of sactipeptides in Escherichia coli: Probing the substrate promiscuity of subtilosin A biosynthesis. ACS Chem. Biol. 2016, 11 (6), 1737.

(14) Burkhart, B. J.; Kakkar, N.; Hudson, G. A.; van der Donk, W. A.; Mitchell, D. A. Chimeric Leader Peptides for the Generation of Non-Natural Hybrid RiPP Products. ACS Cent. Sci. 2017, 3 (6), 629.

(15) Hanwell, M. D.; Curtis, D. E.; Lonie, D. C.; Vandermeersch, T.; Zurek, E.; Hutchison, G. R. Avogadro: an advanced semantic chemical editor, visualization, and analysis platform. J. Cheminform. 2012, 4 (1), 17. (16) Rappe, A. K.; Casewit, C. J.; Colwell, K. S.; Goddard, W. A.; Skiff, W. M. UFF, a full periodic table force field for molecular mechanics and molecular dynamics simulations. J. Am. Chem. Soc. 1992, 114 (25), 10024.

(17) Frisch, M. J.; Trucks, G. W.; Schlegel, H. B.; Scuseria, G. E.; Robb, M. A.; Cheeseman, J. R.; Scalmani, G.; Barone, V.; Petersson, G. A.; Nakatsuji, H.; Li, X.; Caricato, M.; Marenich, A. V.; Bloino, J.; Janesko, B. G.; Gomperts, R.; Mennucci, B.; Hratchian, H. P.; Ortiz, J. V.; Izmaylov, A. F.; Sonnenberg, J. L.; Williams; Ding, F.; Lipparini, F.; Egidi, F.; Goings, J.; Peng, B.; Petrone, A.; Henderson, T.; Ranasinghe, D.; Zakrzewski, V. G.; Gao, J.; Rega, N.; Zheng, G.; Liang, W.; Hada, M.; Ehara, M.; Toyota, K.; Fukuda, R.; Hasegawa, J.; Ishida, M.; Nakajima, T.; Honda, Y.; Kitao, O.; Nakai, H.; Vreven, T.; Throssell, K.; Montgomery Jr., J. A.; Peralta, J. E.; Ogliaro, F.; Bearpark, M. J.; Heyd, J. J.; Brothers, E. N.; Kudin, K. N.; Staroverov, V. N.; Keith, T. A.; Kobayashi, R.; Normand, J.; Raghavachari, K.; Rendell, A. P.; Burant, J. C.; Iyengar, S. S.; Tomasi, J.; Cossi, M.; Millam, J. M.; Klene, M.; Adamo, C.; Cammi, 
R.; Ochterski, J. W.; Martin, R. L.; Morokuma, K.; Farkas, O.; Foresman, J. B.; Fox, D. J. Wallingford, CT, 2016.

(18) Becke, A. D. Density-functional thermochemistry. III. The role of exact exchange. J. Chem. Phys. 1993, 98 (7), 5648.

(19) Stephens, P. J.; Devlin, F. J.; Chabalowski, C. F.; Frisch, M. J. Ab initio calculation of cibrational absorption and circular dichroism spectra using density functional force fields. J. Phys. Chem. 1994, 98 (45), 11623.

(20) Monneau, Y. R.; Ishida, Y.; Rossi, P.; Saio, T.; Tzeng, S.-R.; Inouye, M.; Kalodimos, C. G. Exploiting E. coli auxotrophs for leucine, valine, and threonine specific methyl labeling of large proteins for NMR applications. Journal of biomolecular NMR 2016, 65 (2), 99.

(21) Jang, S.; Imlay, J. A. Hydrogen peroxide inactivates the Escherichia coli Isc iron-sulphur assembly system, and OxyR induces the Suf system to compensate. Mol Microbiol 2010, 78 (6), 1448.

(22) Zhang, Z.; Hudson, G. A.; Mahanta, N.; Tietz, J. I.; van der Donk, W. A.; Mitchell, D. A. Biosynthetic Timing and Substrate Specificity for the Thiopeptide Thiomuracin. Journal of the American Chemical Society 2016, 138 (48), 15511.

(23) Katoh, K.; Standley, D. M. MAFFT multiple sequence alignment software version 7: improvements in performance and usability. Mol. Biol. Evol. 2013, 30 (4), 772.

(24) Lohans, C. T.; Vederas, J. C. Structural characterization of thioether-bridged bacteriocins. J. Antibiot. 2014, 67 (1), 23.

(25) Mundle, S. O. C.; Lacrampe-Couloume, G.; Lollar, B. S.; Kluger, R. Hydrolytic decarboxylation of carboxylic acids and the formation of protonated carbonic acid. J. Am. Chem. Soc. 2010, 132 (7), 2430.

(26) Caruso, A.; Bushin, L. B.; Clark, K. A.; Martinie, R. J.; Seyedsayamdost, M. R. Radical Approach to Enzymatic $\beta$-Thioether Bond Formation. Journal of the American Chemical Society 2019, 141 (2), 990.

(27) Price, M. N.; Dehal, P. S.; Arkin, A. P. FastTree 2--approximately maximum-likelihood trees for large alignments. PLoS One 2010, 5 (3), e9490.

(28) Letunic, I.; Bork, P. Interactive Tree Of Life (iTOL): an online tool for phylogenetic tree display and annotation. Bioinformatics 2007, 23 (1), 127.

(29) Mahanta, N.; Zhang, Z.; Hudson, G. A.; van der Donk, W. A.; Mitchell, D. A. Reconstitution and Substrate Specificity of the Radical S-Adenosyl-methionine Thiazole C-Methyltransferase in Thiomuracin Biosynthesis. J. Am. Chem. Soc. 2017, 139 (12), 4310.

(30) Zhang, Q.; Ortega, M.; Shi, Y.; Wang, H.; Melby, J. O.; Tang, W.; Mitchell, D. A.; van der Donk, W. A. Structural investigation of ribosomally synthesized natural products by hypothetical structure enumeration and evaluation using tandem MS. Proc. Natl. Acad. Sci. USA 2014, 111 (33), 12031.

(31) Wang, J.; Zhang, L.; Teng, K.; Sun, S.; Sun, Z.; Zhong, J. Cerecidins, novel lantibiotics from Bacillus cereus with potent antimicrobial activity. Appl. Environ. Microbiol. 2014, 80 (8), 2633.

(32) Voller, G. H.; Krawczyk, J. M.; Pesic, A.; Krawczyk, B.; Nachtigall, J.; Sussmuth, R. D. Characterization of new class III lantibiotics--erythreapeptin, avermipeptin and griseopeptin from Saccharopolyspora erythraea, Streptomyces avermitilis and Streptomyces griseus demonstrates stepwise N-terminal leader processing. ChemBioChem 2012, 13 (8), 1174. 